\title{
Uma aplicação do FBST no teste de nulidade do parâmetro extra na distribuição de Poisson generalizada
}

Paulo do Canto Hubert Junior

\author{
DisSERTAÇÃO APRESENTADA \\ Instituto de MATEMÁtica E Estatística \\ UNIVERSIDADE DE SÃo PAUlo \\ PARA \\ OBTENÇÃ̃O DO TÍTULO \\ Mestre EM CiÊNCIAS
}

Programa: Mestrado em Estatística

Orientador: Prof. Dr. Júlio Michael Stern 


\section{Agradecimentos}

Em primeiro lugar aos meus pais, Paulo e Maria do Carmo, e minhas irmãs Ana Karina e Ana Paula; sem a ajuda e o apoio deles minha vida seria impossível.

Agradeço também ao Professor Julio Stern, pela paciência e pelo inestimável auxílio na produção da pesquisa.

Ao Professor Carlinhos, um agradecimento especial: sem a sua ajuda, fruto de sua obstinação e dedicação ao ensino da Estatística, eu jamais teria concluído esse programa.

A Cristiane Karcher pela grande ajuda na correção do texto, e pelo gentil empréstimo de sua impressora.

A Keika, Flávio, Guilherme e Filipe, cujas companhias proveram inspiração ininterrupta.

Aos meus colegas Cláudio Queiroz e Luiz Gustavo Martins, pela inestimável ajuda com o código em C++. 


\section{Resumo}

A distribuição de Poisson generalizada acrescenta, à distribuição de Poisson comum, um parâmetro extra, que induz à perda de homogeneidade dos processos estocásticos modelados pela distribuição. Assim, torna-se um modelo útil para utilização em processos de contagem onde a ocorrência dos eventos não é homogênea no tempo ou espaço. Porém, faz-se necessário um procedimento inferencial para o teste desse parâmetro extra.

O FBST (Full Bayesian Signifcance Test) é um teste plenamente bayesiano, que propõe uma medida de evidência para hipóteses precisas (i.e., onde o espaço paramétrico sob a hipótese nula apresenta dimensão menor do que o espaço paramétrico original). O objetivo desse trabalho é o de aplicar o FBST ao teste para o parâmetro extra na distribuição de Poisson generalizada; a hipótese de interesse é, portanto, $H_{0}: \lambda=0$.

No segundo capítulo, a distribuição de Poisson generalizada é definida, e algumas de suas propriedades são apresentadas, bem como são expostos alguns modelos que a utilizam. No terceiro capítulo, o FBST é definido, e sua aplicação ao caso específico dessa dissertação é construída. No quarto capítulo, os algoritmos de geração de números aleatórios são apresentados; o quinto capítulo apresenta os resultados, e o sexto capítulo conclui o trabalho.

Palavras-chave: testes de hipóteses, inferência, inferência bayesiana, processos de contagem, distribuição de Poisson. 


\section{Abstract}

The generalized Poisson distribution adds an extra parameter to the usual Poisson distribution. This parameter induces a loss of homogeneity in the stochastic processes modelled by the distribution, and thus the generalized distribution becomes an useful model for counting processes where the ocurrence of events is not homogeneous in time or space. However, it creates the need for an inferencial procedure, to test for the value of this extra parameter.

The FBST (Full Bayesian Signifcance Test) is a fully bayesian hypotheses testing procedure, which puts forth an evidence measure on sharp hypotheses (where the dimension of the parametric space under the null hypotheses is smaller than that of the full parametric space). The goal of this work is to apply the FBST to the test for the extra parameter of the generalized Poisson distribution. The null hypothesis to be tested is that $H_{0}: \lambda=0$.

In the second chapter, the generalized Poisson distribution is defined, with some of its properties and some models leading to the distribution. In the third chapter, the FBST is defined, and the appllication of the test to the subject of this work is build. The fourth chapter presents the algorithms for random numbers generation; the fifth chapter presents the results, and the sixth chapter concludes the work.

Keywords: hypotheses testing, inference, bayesian inference, counting processes, Poisson distribution. 


\section{Sumário}

Lista de Tabelas $\quad$ vii

$\begin{array}{ll}\text { Lista de Figuras } & \text { viii }\end{array}$

1 Introdução 1

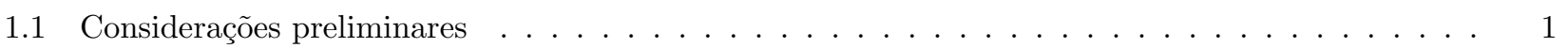

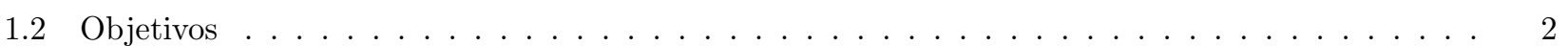



2 Distribuição de Poisson Generalizada $\quad 3$

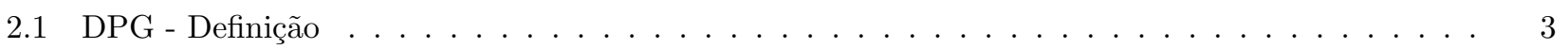

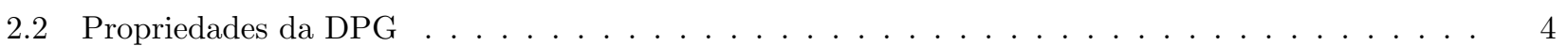



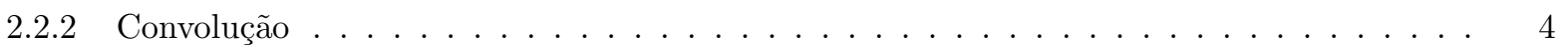

2.2 .3 Função Geradora . . . . . . . . . . . . . . . . . . . . . . . . 5

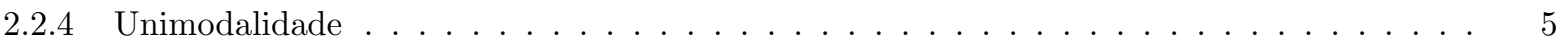

2.2.5 Fórmula de recorrência e probabilidade acumulada $\ldots \ldots \ldots \ldots \ldots \ldots$

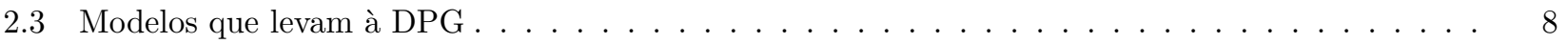

2.3.1 Limite da Binomial Negativa Generalizada . . . . . . . . . . . . . . . . . . 8

2.3.2 Limite da distribuição Quasi-binomial . . . . . . . . . . . . . . . . . . . . . 8

2.3.3 Modelos baseados em equações diferenciais . . . . . . . . . . . . . . . . . . . . . . 9

2.3.4 Processo de Poisson Generalizado . . . . . . . . . . . . . . . . . . . . . . . 11

2.3.5 Distribuição de Clientes em Filas . . . . . . . . . . . . . . . . . . . . . . . . . . . 13



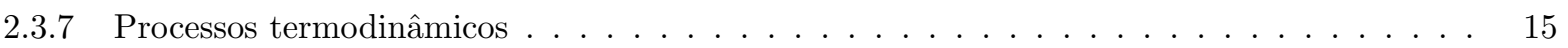




2.4 Momentos de ordem mais alta . . . . . . . . . . . . . . . . . 16

2.4 .2 Assimetria e Curtose . . . . . . . . . . . . . . . . . . . . . . . 19

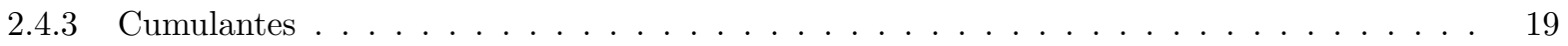

2.4.4 Convergência para a normal . . . . . . . . . . . . . . . . . . . . . . . 19

2.4.5 Convergência para a normal inversa . . . . . . . . . . . . . . . . . . . . . 20

2.4.6 Momentos incompletos . . . . . . . . . . . . . . . . . . . . . . . 20

2.4 Soma de DPGs truncadas . . . . . . . . . . . . . . . . . . . . . . 21

2.4.8 Estatísticas de ordem . . . . . . . . . . . . . . . . . . . . . . . . 21

3 FBST - Full Bayesian Significance Test 23

3.1 Definição do e-valor . . . . . . . . . . . . . . . . . . . . . . . . . . . . . . 24

3.1 .1 Observações sobre o FBST . . . . . . . . . . . . . . . . . . . . 25

3.2 Integração de Monte Carlo . . . . . . . . . . . . . . . . . . . . . . . . . . 25

3.2.1 Importance sampling . . . . . . . . . . . . . . . . . . . . . . . 26

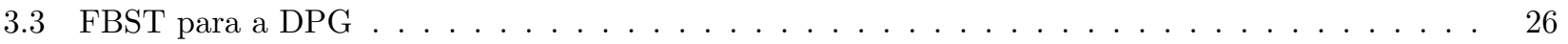

4 Geração de variáveis pseudo-aleatórias 29

4.1 Gerando números da DPG . . . . . . . . . . . . . . . . . . . . . . . . . . 29

4.2 Distribuição para o Importance sampling . . . . . . . . . . . . . . . . . . . . . . 30

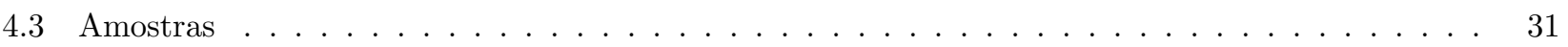

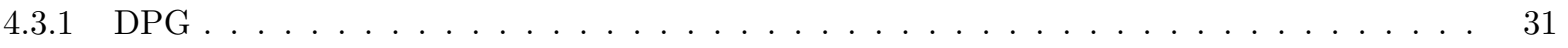

4.3 .2 Importance sampling . . . . . . . . . . . . . . . . . . . . . . . . 34

5 Testes e Resultados 36

5.1 Teste de Consul-Famoye . . . . . . . . . . . . . . . . . . . . . . 36

5.2 Teste de Razão de Verossimilhanças Generalizado (TRVG) . . . . . . . . . . . . . . . . . 36

5.3 Implementação dos testes . . . . . . . . . . . . . . . . . . . . . . . . 37

5.4 Espaço paramétrico . . . . . . . . . . . . . . . . . . . . . . . 37



5.5.1 Valores de evidência sobre o espaço paramétrico . . . . . . . . . . . . . . . . 39

5.5 .2 Desempenho quando $\lambda=\lambda_{0} \ldots \ldots \ldots \ldots \ldots \ldots$

5.5.3 Desempenho para $\lambda \neq 0 \ldots \ldots \ldots \ldots \ldots \ldots$

6 Conclusão 
A Tabelas completas $\quad 49$

B Código Fonte $\quad 62$

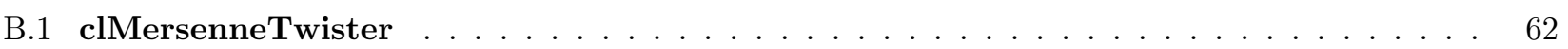

B.1.1 clMersenneTwister.h . . . . . . . . . . . . . . . . . . . 62

B.1.2 clMersenneTwister.cpp . . . . . . . . . . . . . . . . . . . 63

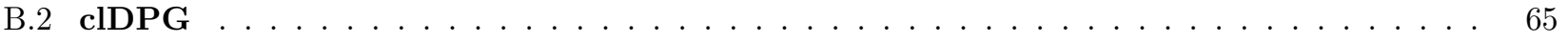

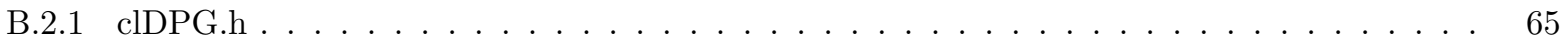

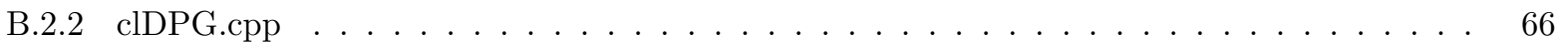

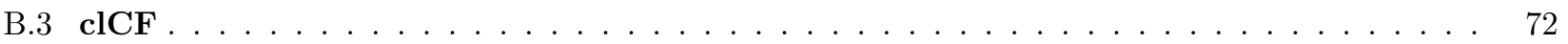

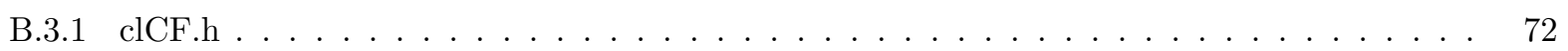

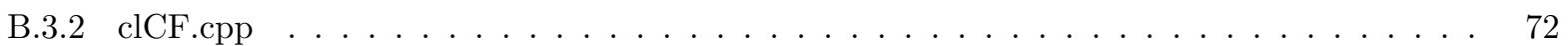

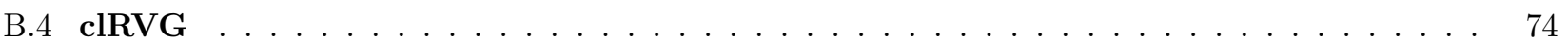

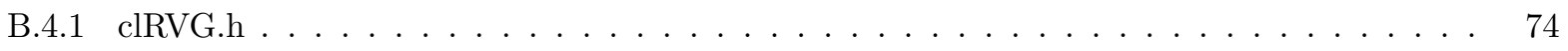

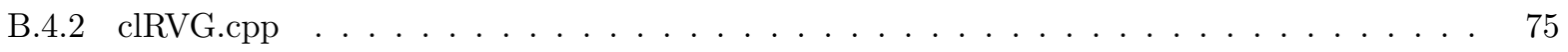

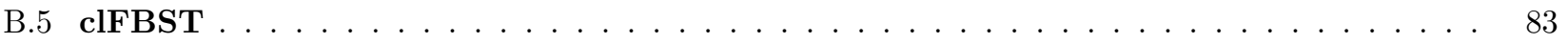

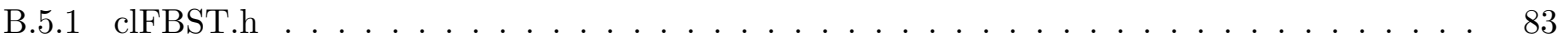

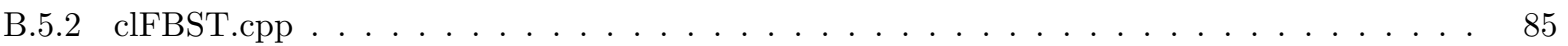




\section{Lista de Tabelas}

5.1 e-,p- e q-valores para $H_{0}$ verdadeira $(\mathrm{N}=20,40 \ldots \ldots \ldots \ldots$

5.2 e-,p- e q-valores para $H_{0}$ verdadeira $(\mathrm{N}=60,100) \ldots \ldots \ldots \ldots$

A.1 E-valor médio $-N=20 \ldots \ldots \ldots \ldots \ldots \ldots$

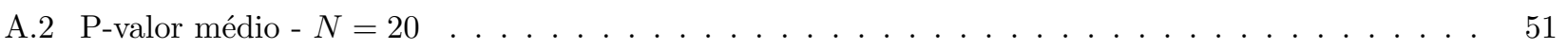

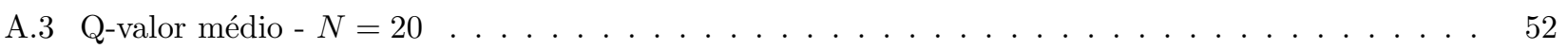

A.4 E-valor médio $-N=40 \ldots \ldots \ldots \ldots \ldots$

A.5 P-valor médio $-N=40 \ldots \ldots \ldots \ldots \ldots \ldots$

A.6 Q-valor médio $-N=40 \ldots \ldots \ldots \ldots \ldots$

A.7 E-valor médio $-N=60 \ldots \ldots \ldots \ldots \ldots \ldots$

A.8 P-valor médio $-N=60 \ldots \ldots \ldots \ldots \ldots \ldots$

A.9 Q-valor médio $-N=60 \ldots \ldots \ldots \ldots \ldots \ldots$

A.10 E-valor médio $-N=100 \ldots \ldots \ldots \ldots \ldots \ldots$

A.11 P-valor médio $-N=100 \ldots \ldots \ldots \ldots \ldots$

A.12 Q-valor médio $-N=100 \ldots \ldots \ldots \ldots \ldots$ 


\section{Lista de Figuras}

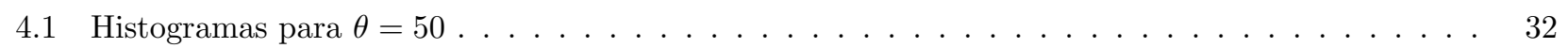

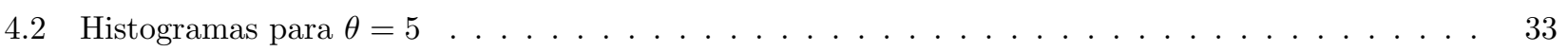

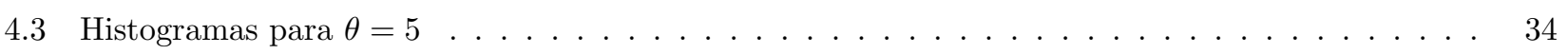



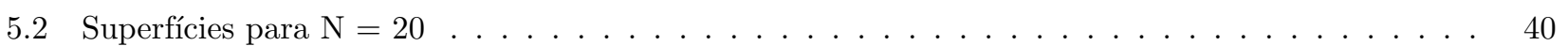

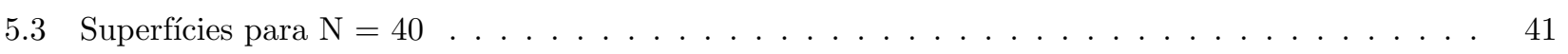

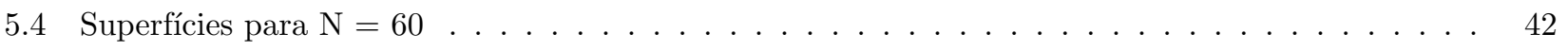



5.6 Valores de evidência em favor da hipótese para $\mathrm{H} 0$ verdadeira $\ldots \ldots \ldots \ldots$. . . . . . . . 44

5.7 Valores de evidência em favor da hipótese para $\theta=50 \ldots \ldots \ldots \ldots$ 


\section{Capítulo 1}

\section{Introdução}

\subsection{Considerações preliminares}

A distribuição de Poisson foi desenvolvida em 1838 pelo matemático e físico francês Siméon Denis Poisson, em seu trabalho Recherches sur la probabilité des jugements en matières criminelles et matière civile. Sua aplicação principal é a modelagem de dados de contagem — tipicamente o número de ocorrências de um determinado evento, num intervalo de tempo ou espaço. Sua função densidade de probabilidade é dada por

$$
P(X=x)=\frac{e^{-\theta} \cdot \theta^{x}}{x !} \quad x=0,1,2, \ldots \quad \theta>0
$$

O único parâmetro da distribuição é o $\theta$, que pode ser interpretado como a taxa de ocorrência dos eventos em questão. Além disso, tanto a média quanto a variância da distribuição são dadas por $\theta$.

Uma hipótese implícita na distribuição de Poisson é a homogeneidade da taxa de ocorrência do evento. Ou seja, admite-se que os processos estocásticos modelados pela Poisson sejam homogêneos, com taxas independentes do ponto particular do tempo ou espaço.

Para algumas aplicações essa hipótese é razoável; há, porém, muitos casos onde esses processos estocásticos dificilmente podem ser considerados homogêneos. Quando observamos, por exemplo, a distribuição de uma colônia de bactérias num dado espaço, notamos com frequência que os organismos tendem a se concentrar mais em certas áreas, do que em outras - isso pode se dar devido à disponibilidade de alimento, à temperatura, ou a comportamentos sociais. Outros casos onde o princípio de homogeneidade é violado podem incluir problemas de acidentes de carro, falhas industriais, incidência de doenças em seres humanos, e ainda outros.

Nesses casos, com a homogeneidade violada, a utilização da distribuição de Poisson produz resultados pouco confiáveis. Em consequência disso, muitos modelos alternativos baseados na distribuição de Poisson original têm sido desenvolvidos - especialmente modelos com $\theta$ variável. Greenwood e Yule [3], por exemplo, assumem que o parâmetro $\theta$ é uma variável aleatória, seguindo a distribuição Gama, o que os leva à distribuição binomial negativa. Outros modelos que surgiram foram o Poisson binomial, Poisson binomial negativa, quasi-Poisson, e por aí afora. Esses modelos surgiram, via de regra, como uma tentativa de modelar a diferença entre média e variância nos dados (não permitida pela Poisson original) ou o aparecimento de valores incomuns de frequências.

Nesse trabalho, vamos nos concentrar na Distribuição de Poisson Generalizada (doravante DPG), conforme desenvolvida por Consul e Jain [4][5][6]. Essa distribuição possui dois parâmetros, $\theta$ e $\lambda$, e apesar de sua forma simples possui grande flexibilidade; a depender do valor de $\lambda$ (positivo ou negativo), a distribuição apresenta sobredispersão ou subdispersão (respectivamente), e a presença desse segundo parâmetro torna desnecessária a hipótese de homogeneidade na ocorrência dos eventos. 
Uma inferência que surge imediatamente como necessária à utilização desse modelo é o teste sobre o parâmetro extra; testando $H_{0}: \lambda=0$ (onde $\lambda$ é o parâmetro extra), testa-se implicitamente se os processos modelados pela distribuição são de fato homogêneos, e assim o modelo usual de Poisson é aplicável, ou se é necessária a utilização do modelo generalizado.

Para essa inferência, será proposta a utilização do FBST (Full Bayesian Significance Test), teste de hipóteses desenvolvido por Pereira e Stern [2] com o objetivo de fornecer uma medida de evidência para hipóteses precisas; o teste apresenta propriedades ótimas para um valor de evidência, especialmente nos casos de hipóteses desse tipo.

\subsection{Objetivos}

O presente trabalho pretende aplicar o Full Bayesian Signicance Test, FBST, ao teste do parâmetro extra na distribuição de Poisson generalizada. Com isso, pretende-se analisar o comportamento do valor de medida fornecido pelo FBST (em comparação a outros testes presentes na literatura) numa situação crítica tanto para a inferência frequentista quanto para a bayesiana usual: o teste de hipóteses precisas.

Para isso, será desenvolvido um programa em $\mathrm{C}++$ que implementa a distribuição de Poisson generalizada, e também os cálculos dos testes. Especialmente para o FBST, será implementado um método de cálculo numérico para integrais (Monte Carlo com importance sampling).

O código fonte utilizado nos cálculos será disponibilizado no Apêndice.

\subsection{Organização do trabalho}

No Capítulo 2, a distribuição de Poisson generalizada é definida, com algumas de suas propriedades e alguns modelos relevantes, seguindo o trabalho de Consul [1]. Em seguida, o Capítulo 3 define o FBST e constrói o teste para a hipótese de interesse deste trabalho. O Capítulo 4 apresenta os algoritmos para geração de variáveis aleatórias; no Capítulo 5 os resultados dos testes são apresentados, e o Capítulo 6 conclui a dissertação.

O Apêndice A apresenta as tabelas com os resultados dos testes, e o Apêndice B contém os códigos-fonte em C++ utilizados nos cálculos. 


\section{Capítulo 2}

\section{Distribuição de Poisson Generalizada}

Nesse capítulo, a distribuição de Poisson generalizada será definida, e algumas de suas propriedades serão apresentadas.

\subsection{Distribuição de Poisson Generalizada - definição}

Seja $X$ uma variável aleatória discreta, que assume somente valores não-negativos. Dizemos que $X$ segue uma DPG se sua distribuição de probabilidades $P_{x}(\theta, \lambda)$ for dada por:

$$
P_{x}(\theta, \lambda)= \begin{cases}\frac{\theta(\theta+x \lambda)^{x-1} \cdot e^{-\theta-x \lambda}}{x !} & x=0,1,2, \ldots \\ 0 & \text { se } x>m, \text { quando } \lambda<0\end{cases}
$$

onde $\theta>0, \max (-1,-\theta / m)<\lambda \leq 1$, e $m(\geq 4)$ é o maior inteiro tal que $\theta+m \lambda>0$ quando $\lambda<0$. O limite mínimo é imposto para assegurar que existam pelo menos cinco pontos do espaço amostral com probabilidade maior do que zero quando $\lambda$ é negativo.

Ao longo desse trabalho, utilizaremos também a seguinte notação

$$
(\theta, \lambda)_{x}=\frac{\theta(\theta+x \lambda)^{x-1}}{x !}
$$

para simplificar as expressões. Dessa forma, a DPG (2.1) pode ser escrita como

$$
P_{x}(\theta, \lambda)=(\theta, \lambda)_{x} \cdot e^{-\theta-x \lambda}
$$

Comparando a forma da DPG em (2.1) com a distribuição de Poisson usual (1.1), vemos que em (2.1) o parâmetro $\theta$ foi substituído (embora não exatamente) por $\theta+x \lambda$; ou seja, a grosso modo, a DPG é uma Poisson com uma taxa de ocorrência que depende de $\mathrm{x}$, o ponto onde se avalia a probabilidade. Além disso, nota-se que fazendo $\lambda=0$, a DPG reduz-se à Poisson - todos os resultados obtidos para a DPG, portanto, podem ser estendidos para a Poisson fazendo-se $\lambda=0$.

Nos casos onde $\lambda$ é negativo, a distribuição é truncada no ponto $\mathrm{m}$, pois da definição temos que $P_{x}(\theta, \lambda)=0$ para todo $x>m$. Por conta disso, a soma

$$
F_{m}(\theta, \lambda)=\sum_{x=0}^{m} P_{x}(\theta, \lambda)
$$


não será igual a 1. Portanto a DPG deve ser utilizada com cautela quando $\lambda$ for negativo; a literatura muitas vezes recomenda uma normalização dos valores de $P_{x}(\theta, \lambda)$, multiplicando cada probabilidade por $\left[F_{m}(\theta, \lambda)\right]^{-1}$. Consul e Shoukri [7] fizeram um estudo detalhado dos efeitos dessa normalização, tanto sobre as probabilidades quanto sobre os valores de média e variância da DPG, e concluíram que para valores de $m$ grandes o erro incorrido quando da não utilização da constante $\left[F_{m}(\theta, \lambda)\right]^{-1}$ é muito pequeno. Assim, para a maior parte dos casos práticos de aplicação da DPG, a normalização é desnecessária.

Em muitas situações, é conveniente escrever o parâmetro $\lambda$ como uma função linear de $\theta$. Fazendo $\lambda=\alpha \theta$, temos o modelo da DPG restrita:

$$
\begin{aligned}
P_{x}(\theta, \alpha \theta) & =\frac{(1+x \alpha)^{x-1} \theta^{x} e^{-\theta-x \alpha \theta}}{x !}, \quad x=0,1,2, \ldots \\
& =(1, \alpha)_{x}\left(\theta e^{-\alpha \theta} e^{-\theta}\right)
\end{aligned}
$$

Para esse modelo, temos que $\max \left(-\theta^{-1},-m^{-1}\right)<\alpha<\theta^{-1}$ e, novamente, $P_{x}(\theta, \alpha \theta)=0$ para $x>m$ quando $\alpha<0$.

\subsection{Propriedades da Distribuição de Poisson Generalizada}

\subsubsection{Média e Variância}

Seja $X$ uma DPG restrita com parâmetros $(\theta, \alpha \theta)$. A média e a variância de $X$ são dadas por:

$$
E(X)=\frac{\theta}{1-\alpha \theta} \quad \operatorname{Var}(X)=\frac{\theta}{(1-\alpha \theta)^{3}}
$$

No caso não-restrito, se $X$ for uma DPG com parâmetros $(\theta, \lambda)$, sua média e variância serão

$$
E(X)=\frac{\theta}{1-\lambda} \quad \operatorname{Var}(X)=\frac{\theta}{(1-\lambda)^{3}}
$$

analogamente ao caso restrito.

\subsubsection{Convolução}

Uma propriedade importante da DPG, análoga à Poisson, é a propriedade de convolução, que enunciamos da seguinte maneira:

Teorema 2.2.2.1. Sejam $X_{1}$ e $X_{2}$ duas variáveis aleatórias independentes com distribuição de Poisson generalizada, de parâmetros $\left(\theta_{1}, \lambda\right)$ e $\left(\theta_{2}, \lambda\right)$, respectivamente. Então, a soma $X_{1}+X_{2}$ também seguirá uma $D P G$, com parâmetros $\left(\theta_{1}+\theta_{2}, \lambda\right)$.

A demonstração do teorema será omitida, mas pode ser encontrada em Consul [1]. O teorema pode ser generalizado para a soma de $n$ variáveis com DPG independentes. 


\subsubsection{Função Geradora}

A Função Geradora de Probabilidade (FGP) de uma variável aleatória $X$ é definida como $E\left(u^{X}\right)$, e será denotada daqui por diante como $G_{X}(u)$. Para $X \sim \operatorname{DPG}(\theta, \lambda)$, temos

$$
G_{X}(u)=e^{\theta(t-1)}, \quad \text { onde } t=u e^{\lambda(t-1)}
$$

\subsubsection{Unimodalidade}

Uma característica importante da DPG para esse trabalho é a unimodalidade da distribuição. Nessa seção vamos demonstrar essa propriedade para a $\operatorname{DPG}(\theta, \lambda)$.

Para isso, vamos precisar do seguinte lema, demonstrado por Steutel e Harn [8]:

Lema 2.2.4.1. Seja $\left\{P_{x}\right\}_{0}^{\infty}$ uma distribuição de probabilidade definida para inteiros não-negativos, com FGP dada por $G_{x}(u)$. Além disso, seja

$$
\frac{d}{d u} \log G_{x}(u)=R(u)=\sum_{k=0}^{\infty} r_{k} u^{k}, \quad r_{k} \geq 0
$$

Então, a distribuição $\left\{P_{x}\right\}_{0}^{\infty}$ será unimodal se $\left(r_{k}\right)_{0}^{\infty}$ for não-crescente, e se adicionalmente $r_{0} \leq 1,\left\{P_{x}\right\}_{0}^{\infty}$ será não-decrescente.

Vamos agora enunciar a unimodalidade da DPG:

Teorema 2.2.4.1. Os modelos DPG são unimodais para todos os valores de $\theta$ e $\lambda$. A moda está no ponto $x=0$ se $\theta e^{-\lambda}<1$ e nos pontos duais $x=0$ e $x=1$ quando $\theta e^{-\lambda}=1$. Para $\theta e^{-\lambda}>1$, a moda está em algum ponto $x=M$ tal que

$$
\left(\theta-e^{\lambda}\right)\left(e^{\lambda}-2 \lambda\right)^{-1}<M<a
$$

onde a é o menor valor de $M$ que satisfaz

$$
\lambda^{2} M^{2}+M\left[2 \lambda \theta-(\theta+2 \lambda) e^{\lambda}\right]+\theta^{2}>0
$$

Demonstração. Conforme vimos anteriormente, a DPG tem FGP dada por

$$
G_{x}(u)=\sum_{x=0}^{\infty} u^{x} P_{x}(\theta, \lambda)=e^{\theta(t-1)}
$$

onde

$$
t=u e^{\lambda(t-1)}=\sum_{n=1}^{\infty} e^{-n \lambda} \frac{(n \lambda)^{n-1}}{n !} u^{n}=H(u)
$$

Derivando o logaritmo de $G_{x}(u)$ com respeito a $u$, e aplicando o Lema 1.2.4.1 acima, temos: 


$$
\begin{aligned}
R(u) & =\sum_{k=0}^{\infty} r_{k} u^{k}=\theta \frac{d t}{d u}=\theta \frac{d H(u)}{d u} \\
& =\theta \sum_{n=1}^{\infty} e^{-n \lambda} \frac{(n \lambda)^{n-1}}{(n-1) !} u^{n-1}=\theta \sum_{k=0}^{\infty} e^{-\lambda-k \lambda} \frac{[(k+1) \lambda)]^{k}}{k !} u^{k}
\end{aligned}
$$

Portanto

$$
\begin{aligned}
\frac{r_{k}}{r_{k-1}} & =\frac{e^{-\lambda(k+1)}[\lambda(k+1)]^{k} / k !}{e^{-k \lambda}(\lambda k)^{k-1} /(k-1) !} \\
& =\lambda e^{-\lambda}\left(1+\frac{1}{k}\right)^{k} \\
& \leq \lambda e^{-\lambda+1} \leq 1
\end{aligned}
$$

pois $\left(1+k^{-1}\right)^{j} \leq e$ e $1+x<e^{x}$ para todo $x \in \mathbf{R}$. Portanto, $\left[r_{k}\right]_{0}^{\infty}$ é não-crescente e, pelo Lema 1.2.4.1, $\left\{P_{x}(\theta, \lambda)\right\}_{0}^{\infty}$ é unimodal para todos os valores de $\theta$ e $\lambda$. Além disso, temos que quando $P_{1}(\theta, \lambda) / P_{0}(\theta, \lambda)=\theta e^{-\lambda}=r_{0} \leq 1$, a sequência $\left\{P_{x}(\theta, \lambda)\right\}$ é também não-crescente. Assim, a moda estará em $x=0$ quando $\theta e^{-\lambda}<1$ e nos pontos duais $x=0$ e $x-1$ quando $\theta e^{-\lambda}=1$. Agora, fazendo $\theta e^{-\lambda}>1$, a moda estará no ponto $x=M$.

O quociente entre duas probabilidades sucessivas da DPG é:

$$
\frac{P_{x+1}(\theta, \lambda)}{P_{x} \theta, \lambda}=\frac{(\theta+x \lambda+\lambda)^{x}}{(\theta+x \lambda)^{x-1}} \frac{e^{-\lambda}}{x+1}
$$

Sendo M a moda, o quociente acima nos fornece as seguintes inequações:

$$
\frac{P_{M+1}(\theta, \lambda)}{P_{M}(\theta, \lambda)}=\frac{(\theta+\lambda M+\lambda)^{M} e^{-\lambda}}{(M+1)(\theta+\lambda M)^{M-1}}<1
$$

e

$$
\frac{P_{M}(\theta, \lambda)}{P_{M-1}(\theta, \lambda)}=\frac{(\theta+\lambda M)^{M-1} e^{-\lambda}}{M(\theta+\lambda M-\lambda)^{M-2}}>1
$$

Pela inequação (2.13)

que é verdadeira se

$$
(M+1) e^{\lambda}>(\theta+\lambda M)\left(1+\frac{\lambda}{\theta+\lambda M}\right)^{M}
$$

$$
(M+1) e^{\lambda}>(\theta+\lambda M)\left(1+\frac{\lambda M}{\theta+\lambda M}\right)=\theta+2 \lambda M
$$

Da inequação acima deduz-se

$$
M>\left(\theta-e^{\lambda}\right)\left(e^{\lambda}-2 \lambda\right)^{-1}
$$

Pela inequação (2.14), 


$$
\frac{(\theta+\lambda M) e^{-\lambda}}{M}>\left(1-\frac{\lambda}{\theta+\lambda M}\right)^{M-2}
$$

que é verdade se

$$
\frac{(\theta+\lambda M) e^{-\lambda}}{M}>1-(M-2) \lambda(\theta+\lambda M)^{-1}=\frac{\theta+2 \lambda}{\theta+\lambda M}
$$

ou seja,

$$
(\theta+\lambda M)^{2}>M(\theta+2 \lambda) e^{\lambda}
$$

que é equivalente à inequação (2.11) e dá o limite superior da moda.

Corolário: Quando $\lambda=0$, os limites da moda são

$$
\theta-1<M<\theta
$$

Está assim demonstrada a unimodalidade da DPG, para quaisquer valores de $\theta$ e $\lambda$. Essa propriedade garante a existência de um máximo único para a distribuição, o que vai facilitar a otimização necessária para cálculo do FBST, conforme se verá.

\subsubsection{Fórmula de recorrência e probabilidade acumulada}

O cálculo direto de $P_{x}(\theta, \lambda)$ pode se tornar computacionalmente difícil, à medida que, quando $x$ toma valores grandes, $e^{-\theta-\lambda x} / x$ ! torna-se muito pequeno enquanto $\theta(\theta+x \lambda)^{x-1}$ cresce. A equação seguinte fornece uma relação de recorrência entre os valores da probabilidade da DPG, e pode ser útil para o cálculo das probabilidades exatas:

$$
P_{x}(\theta, \lambda)=e^{-\lambda}\left(\lambda+\frac{\theta}{x}\right)\left(1+\frac{\lambda}{\theta+x \lambda-\lambda}\right)^{x-2} P_{x-1}(\theta, \lambda)
$$

As probabilidades cumulativas da DPG são definidas pela $F_{m}(\theta, \lambda)$ em $(2.4)$ e pela soma da cauda:

$$
Q_{x}(\theta, \lambda)=\sum_{i=x+1}^{\infty} P_{i}(\theta, \lambda)
$$

$F_{x}$ e $Q_{x}$ estão relacionadas por

$$
F_{x}(\theta, \lambda)+Q_{x}(\theta, \lambda)=1
$$




\subsection{Modelos que levam à DPG}

A Distribuição de Poisson Generalizada, conforme vimos na seção anterior, tem um forma bastante simples, dependendo apenas de 2 parâmetros, e é matematicamente tratável, como veremos.

O objetivo dessa seção é listar alguns modelos aplicados, relacionados a problemas ou situações reais de análise, que de alguma maneira convergem para a DPG, ou que tem a DPG como uma ferramenta útil.

\subsubsection{Limite da Binomial Negativa Generalizada}

A distribuição Binomial Negativa Generalizada é útil para a modelagem de diferentes problemas probabilísticos. Problemas de teoria das filas, o problema do lançamento de duas moedas, da probabilidade de ruína do jogador num modelo de passeio aleatório associado ao jogo de roleta, a probabilidade de ruína de um jogador que aposta em cavalos, todos de alguma forma culminam na distribuição Binomial Negativa Generalizada. Essa distribuição discreta é definida por:

$$
P(X=x)=\frac{n}{n+\beta x}\left(\begin{array}{c}
n+\beta x \\
x
\end{array}\right) \rho^{x}(1-\rho)^{n+\beta x-x}, \quad x=0,1,2, \ldots
$$

onde $0<\rho<1$ e $1 \leq \beta \leq \rho^{-1}$ para $n>0$ e quando $\beta=0$. O parâmetro $n$ é um inteiro positivo.

Manipulando algebricamente a expressão de $P(X=x)$ usando o fato (Abramowitz e Stegun [9])

$$
\frac{\Gamma(z+a)}{\Gamma(z+b)}=z^{a-b}\left[1+\frac{(a-b)(a+b-1)}{2 z}+o\left(z^{-2}\right)\right]
$$

temos que

$$
P(X=x)=P_{x}(\theta, \lambda)\left[1+\frac{x \theta}{n}-\frac{x^{2} \lambda^{2}}{2 \beta}-\frac{\theta^{2}}{2 n}-\frac{x(x-1)}{2(n+\beta x)}\right]+o\left(n^{-2}\right)
$$

Vê-se a partir da equação que a distribuição Binomial Negativa Generalizada, quando $n$ e $\beta$ são grandes e $\rho$ pequeno de modo que $n \rho=\theta$ e $\beta \rho=\lambda$, converge para uma DPG de parâmetros $(\theta, \lambda)$, e o erro de primeira ordem dessa aproximação é dado por $(x \theta / 2 n)\left(2-\lambda-\theta / x-(x-1)(\theta+x \beta \theta / n)^{-}\right) P_{x}(\theta, \lambda)$.

\subsubsection{Limite da distribuição Quasi-binomial}

Em Consul[10], aparece a definição da distribuição quasi-binomial, dada por:

$$
P(X=x)=\left(\begin{array}{l}
n \\
x
\end{array}\right)\left(\frac{a}{a+x \alpha}\right)\left(\frac{a+x \alpha}{a+b+n \alpha}\right)^{x}\left(\frac{b+n \alpha-x \alpha}{a+b+n \alpha}\right)^{n-x}
$$

para $x=0,1,2, \ldots, n$. Usando a transformação $a(a+b+n \alpha)^{-1}=\rho$ e $\alpha(a+b+n \alpha)^{-1}=\phi$, podemos escrever a quasi-binomial da forma mais conveniente:

$$
P(X=x)=\left(\begin{array}{l}
n \\
x
\end{array}\right) \rho(\rho+x \phi)^{x-1}(1-\rho-x \phi)^{n-x}, \quad x=0,1, \ldots, n
$$

onde $\rho+n \phi \leq 1$ e $0<\rho<1$.

Se fizermos $\rho \rightarrow 0^{+}, \phi \rightarrow 0^{ \pm}$, e $n \rightarrow \infty$, tal que $n \rho=\theta$ e $n \phi=\lambda$, reescrevemos a probabilidade acima como 


$$
P(X=x)=\frac{\theta(\theta+x \lambda)^{x-1}}{x !} \frac{n ! n^{-x}}{(n-x) !}\left(1-\frac{\theta+x \lambda}{n}\right)^{n-x}
$$

Por fim, substituindo $n ! n^{-x} /(n-x)$ ! pela sua expansão, e expressando o último fator da equação acima na forma $e^{-\theta-x \lambda}$, temos:

$$
P(X=x)=P_{x}(\theta, \lambda)\left[1+\frac{1}{2} \frac{x(\theta+x \lambda)-(\theta+x \lambda)^{2}-x(x-1)}{n}\right]+o\left(n^{-2}\right)
$$

e portanto temos que a distribuição quasi-binomial em (2.22), quando $n \rightarrow \infty, \rho \rightarrow 0$ e $\phi \rightarrow 0$ de modo que $n \rho=\theta$ e $n \phi=\lambda$, converge para uma DPG de parâmetros $\theta$ e $\lambda$.

\subsubsection{Modelos baseados em equações diferenciais}

Existem diversos problemas, especialmente na biologia, que podem ser modelados probabilisticamente como o problema de bolas em urnas. Por exemplo a distribuição de bactérias ou micro-organismos em um determinado espaço, a migração de pássaros e aves, a formação de grupos de animais numa área qualquer, e assim por diante.

Analisando um problema dessa espécie, Tukey [11] partiu da hipótese de que a probabilidade de encontrar $x$ bolas em uma urna, $\Pi_{x}$, é função do número médio de bolas na urna, $\lambda$, e de tal maneira que $\Pi_{0}=1$ quando $\lambda=0$. Indo mais além e assumindo que $d \Pi_{0} / d \lambda=-\Pi_{0}$ e $d \Pi_{x} / d \lambda=\Pi_{x-1}$ para $x \geq 1$, ele demonstrou nesse trabalho que a distribuição das bolas nas urnas tem que ser a Poisson.

Analogamente, vamos assumir que a distribuição de probabilidade das bolas nas urnas depende da média do número de bolas na urna $\mu(\theta, \lambda)$, que dessa vez depende de dois parâmetros. Portanto, a probabilidade de encontrarmos $x$ bolas na urna deve ser função de $\theta, \lambda$ e $x$, e será doravante denominada $M_{x}(\theta, \lambda)$. Alterando o valor de cada um dos dois parâmetros (Consul [1]), podemos descrever dois modelos que generalizam o resultado de Tukey.

\section{Modelo I}

Teorema 2.3.3.1. Se a média $\mu(\theta, \lambda)$ da distribuição das bolas em urnas aumentar devido a um acréscimo de $\Delta \theta$ no parâmetro $\theta$ (i.e., o novo parâmetro será $\theta+\Delta \theta$ ) de tal modo que

$$
\frac{d M_{0}(\theta, \lambda)}{d \theta}=-M_{0}(\theta, \lambda)
$$

$e$

$$
\frac{d M_{x}(\theta, \lambda)}{d \theta}=-M_{x}(\theta, \lambda)+M_{x-1}(\theta+\lambda, \lambda)
$$

para todos os valores inteiros positivos de $x$, com as condições iniciais $M_{0}(0, \lambda)=1$ e $M_{x}(0, \lambda)=0$ para $x>0$, então o modelo probabilístico $M_{x}(\theta, \lambda)$ é a $D P G P_{x}(\theta, \lambda)$.

Demonstração. A equação (2.25) é uma equação diferencial linear com solução geral dada por $M_{0}(\theta, \lambda)=C_{0} e^{-\theta}$. E pela condição inicial $M_{0}(0, \lambda)=1, C_{0}=1$. Portanto

$$
M_{0}(\theta, \lambda)=e^{-\theta}=P_{0}(\theta, \lambda)
$$

Usando o resultado acima em (2.26) quando $x=1$, temos 


$$
\frac{d M_{1}(\theta, \lambda)}{d \theta}+M_{1}(\theta, \lambda)=M_{0}(\theta+\lambda, \lambda)=e^{-\theta-\lambda}
$$

A equação acima é uma equação diferencial linear similar a que nos deu o valor de $M_{0}(\theta, \lambda)$. Portanto, resolvendo a equação e utilizando a condição inicial $M_{1}(0, \lambda)=0$, temos

$$
M_{1}(\theta, \lambda)=\theta e^{-\theta-\lambda}=P_{1}(\theta, \lambda)
$$

Fazendo $x=2$ em (2.26) e usando o resultado acima, chegamos à seguinte equação diferencial linear:

$$
\frac{d M_{2}(\theta, \lambda)}{d \theta}+M_{2}(\theta, \lambda)=(\theta+\lambda) e^{-\theta-2 \lambda}
$$

cuja solução geral é

$$
M_{2}(\theta, \lambda)=\left(\frac{\theta^{2}}{2}+\lambda \theta\right) e^{-\theta-2 \lambda}+C_{2} e^{-\theta}
$$

Novamente, utilizando a condição inicial $M_{2}(0, \lambda)=0$ chegamos em $C_{2}=0$ e assim

$$
M_{2}(\theta, \lambda)=\frac{\theta(\theta+2 \lambda) e^{-\theta-2 \lambda}}{2 !}=P_{2}(\theta, \lambda)
$$

Agora para $x=3$, partindo da equação (2.26) e utilizando o resultado acima

$$
\begin{aligned}
\frac{d M_{3}(\theta, \lambda)}{d \theta}+M_{3}(\theta, \lambda) & =\frac{(\theta+\lambda)(\theta+3 \lambda) e^{-\theta-3 \lambda}}{2 !} \\
& =\frac{\left[(\theta+3 \lambda)^{2}-2 \lambda(\theta+3 \lambda)\right] e^{-\theta-3 \lambda}}{2 !}
\end{aligned}
$$

A solução geral para a equação acima é dada por

$$
M_{3}(\theta, \lambda)=e^{-\theta-3 \lambda} \frac{(\theta+3 \lambda)^{3} / 3-\lambda(\theta+3 \lambda)^{2}}{2 !}+C_{3} e^{-\theta}
$$

da condição inicial $M_{3}(0, \lambda)=0, C_{3}=0$ e portanto:

$$
M_{3}(\theta, \lambda)=e^{-\theta-3 \lambda} \frac{\theta(\theta+3 \lambda)^{2}}{3 !}=P_{3}(\theta, \lambda)
$$

Portanto, $M_{x}(\theta, \lambda)=P_{x}(\theta, \lambda)$ para $x=0,1,2,3$. A partir disso, podemos facilmente demonstrar por indução que:

$$
M_{x}(\theta, \lambda)=\frac{e^{-\theta-x \lambda} \theta(\theta+x \lambda)^{x-1}}{x !}=P_{x}(\theta, \lambda)
$$




\section{Modelo II}

Teorema 2.3.3.1. Se a média $\mu(\theta, \lambda)$ da distribuição de probabilidade das bolas na urna cresce a partir de um acréscimo de $\Delta \lambda$ em seu segundo parâmetro (que passa a ser $\lambda+\Delta \lambda$ ), de tal modo que

$$
\frac{d M 0(\theta, \lambda)}{d \lambda}=0
$$

$e$

$$
\frac{d M_{x}(\theta, \lambda)}{d \lambda}=-x M_{x}(\theta, \lambda)+\frac{(x-1) \theta}{\theta+\lambda} M_{x-1}(\theta+\lambda, \lambda)
$$

para todos os valores inteiros positivos de $x$, com as condições iniciais $M_{x}(\theta, 0)=e^{-\theta} \theta^{x} / x !$ para todo $x$, então o modelo probabilístico $M_{x}(\theta, \lambda)$ é a $D P G P_{x}(\theta, \lambda)$.

A demonstração desse teorema é obtida por método análogo ao anterior.

A DPG, portanto, pode surgir como modelo probabilístico em problemas de bolas em urnas, com algumas características específicas.

\subsubsection{Processo de Poisson Generalizado}

Processos de Poisson são processos estocásticos de contagem, onde $N(t)$, o número de ocorrências de um dado evento até o instante $t$, tem distribuição de Poisson com parâmetro $\theta$. Nessa seção, vamos analisar uma generalização do processo de Poisson que descreve a situação de pontos em uma linha de acordo com uma lei probabilística, e que obedece a alguns axiomas. Vamos mostrar que esse processo generalizado terá como distribuição a DPG.

Seja $t$ um intervalo de tempo qualquer e $N(t)$ o número de ocorrências de um evento $E$ no intervalo de duração $t$. Então $N(t)$ será uma variável aleatória discreta com valores $0,1,2, \ldots$ Definimos $g(k, t)$ como a probabilidade $P(N(t)=k)$ de modo que $\sum_{k=0}^{\infty} g(k, t)=1$. Assumimos que o processo $\{N(t)\}$ está em estado de equilíbrio, e que ele satisfaz os seguintes axiomas:

Axioma 1: O processo começa no instante 0 do estado $E_{0}$. Ou seja, $N(0)=0$ e $P(N(0)=0)=g(0,0)=1$. Ainda, $g(x, 0)=0$ para $x>0$.

Axioma 2: Para todo $t>0$

$$
0<P(N(t)>0)<1
$$

Axioma 3: Transições diretas do estado $E_{j}$ só são possíveis para o estado $E_{j+1}$. Em outras palavras, para qualquer $t>0$, em intervalos $h$ suficientemente pequenos no máximo um evento pode ocorrer. Ou seja

$$
P(N(h) \geq 2)=\sum_{x=2}^{\infty} g(x, h)=o(h)
$$

Lembrando que $o(h)$ representa uma função de $h$ tal que $\lim _{h \rightarrow 0}[o(h) / h]=0$. 
Axioma 4: A probabilidade de uma única ocorrência num intervalo pequeno $h$ depende não apenas de $h$, mas também de $t$, do estado $x$ onde o processo se encontra em $t$, e do estado resultante dessa única ocorrência, e essa probabilidade é dada por:

$$
P(N(t+h)-N(t)=0 \mid N(t)=x)=1-(\theta+x \lambda) h+o(h)
$$

e

$$
P(N(t+h)-N(t)=1 \mid N(t)=x-1)=\frac{(\theta+(x-1) \lambda) h e^{-\lambda t}}{\left(1-\lambda(\theta+x \lambda)^{-1}\right)^{x-1}}+o(h)
$$

De acordo com esses axiomas, a probabilidade $g(0, t+h)$ de termos 0 ocorrências num intervalo de tamanho $t+h$ é igual ao produto da probabilidade $g(0, t)$ de 0 ocorrências num intervalo $t$ pela probabilidade $[1-\theta h-o(h)]$ de termos 0 ocorrências num intervalo disjunto de tamanho $h$. Portanto

$$
g(0, t+h)=g(0, t)[1-\theta h-o(h)]
$$

Simplificando a expressão acima e fazendo $h \rightarrow 0$ temos

$$
D_{t}[g(0, t)]+\theta g(0, t)=0
$$

A solução da equação diferencial acima é $g(0, t)=C_{0} e^{-\theta t}$ e a condição inicial $g(0,0)=1$ implica em que $C_{0}=1$. Portanto,

$$
g(0, t)=e^{-\theta t}
$$

Quando $x$ é um inteiro positivo, os axiomas que enunciamos implicam que $g(x, 0)=0$ e

$$
g(x, t+h)=g(x, y)[1-(\theta+x \lambda) h-o(h)]+g(x-1, t)\left[\frac{(\theta+(x-1) \lambda) h e^{-\lambda t}}{\left(1-\lambda(\theta+x \lambda)^{-1}\right)^{x-1}}+\frac{o(h)}{h}\right]
$$

O que leva a

$$
\frac{g(x, t+h)-g(x, t)}{h}+(\theta+\lambda x) g(x, t)=g(x-1, t) \frac{[\theta+(x-1) \lambda] e^{-\lambda t}}{\left[1-\lambda(\theta+x \lambda)^{-1}\right]^{x-1}}+\frac{o(h)}{h}
$$

Levando $h \rightarrow 0$ obtemos a seguinte equação diferencial:

$$
D_{t}[g(x, t)]+(\theta+\lambda x) g(x, t)=\frac{[\theta+(x-1) \lambda] e^{-\lambda t}}{\left[1-\lambda(\theta+x \lambda)^{-1}\right]^{x-1}} g(x-1, t)
$$

para $x=1,2,3, \ldots$

Fazendo $x=1$, a equação anterior, juntamente com (2.37), resulta na equação diferencial

$$
D_{t}[g(1, t)]+(\theta+\lambda) g(1, t)=\theta e^{-\lambda t-\theta t}
$$

cuja solução geral é $g(1, t)=\theta t e^{-\theta t-\lambda t}+C_{1} e^{-\theta t-\lambda t}$. A condição de contorno $g(1,0)=0$ resulta em que $C_{1}=0$, e portanto 


$$
g(1, t)=\theta t e^{-\theta t-\lambda t}
$$

Fazendo agora $x=2,3, \ldots$, e utilizando as condições de contorno $g(x, 0)=0$, é possível mostrar por indução que

$$
g(x, t)=\frac{\theta(\theta+\lambda x)^{x-1} t^{x} e^{-\theta t-x \lambda t}}{x !}, \quad x=2,3,4, \ldots
$$

E, portanto, que o processo de contagem $N(t)$ num intervalo de tamanho t - i.e., o processo estocástico $\{\mathrm{N}(\mathrm{t})\}$ - segue uma DPG com parâmetros $(\theta, \lambda)$.

\subsubsection{Distribuição de Clientes em Filas}

Seja $g(s)$ a FGP do número de clientes chegando a um balcão de atendimento para serem atendidos, e $X$ uma outra variável aleatória indicando o número de clientes esperando na fila antes do início do trabalho do atendente. A FGP de $X$ será denotada por $f(s)$.

Se assumimos que o tempo de atendimento é constante e igual para todos os clientes, Consul e Shenton [12] mostraram que o número de clientes atendidos em qualquer período de funcionamento do balcão de atendimento será uma variável aleatória $Y$ seguindo uma distribuição Lagrangiana dada por

$$
P(Y=y)=\left.(y !)^{-1} D_{s}^{-1}\left[(g(s))^{y} D_{s} f(s)\right]\right|_{s=0}, \quad y \in T
$$

onde $T$ é um subconjunto dos inteiros não-negativos.

Tomando $f(s)=\exp [\theta(s-1)]$ e $g(s)=\exp [\lambda(s-1)]$ e simplificando a expressão acima, temos que:

$$
P(Y=y)=P_{y}(\theta, \lambda)
$$

Ou seja, a DPG descreve a distribuição de probabilidade do número de clientes atendidos quando os clientes chegam de acordo com uma Poisson e o número de clientes esperando na fila antes do serviço começar também é uma Poisson.

\subsubsection{Distribuição da Progenia num Processo de Ramificação de Galton-Watson}

Um processo de ramificação, usualmente, descreve a situação onde uma determinada característica é transmitida para alguns membros de um grupo a partir de um indivíduo ou grupo "infectado"num ponto inicial do tempo. Em seguida, os indivíduos que herdaram a característica a transmitem para a próxima geração, e assim por diante.

Shoukri e Consul [13] mostraram que a DPG pode ser usada para processos de ramificação em todas as situação onde pelo menos uma dessas condições esteja satisfeita

1. O número total de unidades ou indivíduos no grupo é grande.

2. A probabilidade de um indivíduo adquirir a característica (infectar-se) é pequena.

3. Cada indivíduo infectado torna-se um transmissor por um curto período de tempo.

4. O número de membros no grupo onde cada transmissor está propenso a transmitir a característica também é grande. 
Em cada um desses casos, o número total de indivíduos infectados (progenia) seguirá uma DPG.

Definimos $X_{0}$ como o número original de indivíduos infectados. Por conta das condições 1 e $2, X_{0}$ deve seguir uma Poisson. Assim, sua FGP será:

$$
f(t)=E\left[t^{X_{0}}\right]=\exp [\theta(t-1)]
$$

Seja $v$ o número de indivíduos que cada ancestral (i.e., um indivíduo originalmente infectado) infecta na primeira geração. Pelas condições 3 e $4, v$ também será uma Poisson, com FGP dada por $g(t)=\exp [\lambda(t-1)]$.

Vamos denotar por $X_{0}, X_{1}, X_{2}, \ldots$ o número de indivíudos infectados em cada geração. Podemos assumir que a distribuição de cada $X_{i}$ é a mesma - basta considerar a geração $i-1$ como sendo a primeira. Vamos definir $g_{n}(s)=E\left[s^{X_{n}}\right]$. Além disso, se assumimos que $X_{0}=1$ com probabilidade 1 (i.e., o processo começa com um único indivíduo infectado), temos que $g_{0}(s)=s$ e $g_{1}(s)=g(s)$. Portanto, para $n=2,3, \ldots$

$$
\begin{aligned}
g_{n+1}(s) & =\sum_{k=0}^{\infty} P\left(X_{n+1}=k\right) s^{k} \\
& =\sum_{k=0}^{\infty} s^{k} \sum_{j=0}^{\infty} P\left(X_{n+1}=k \mid X_{n}=j\right) P\left(X_{n}=j\right) \\
& =\sum k=0^{\infty} P\left(X_{n}=j\right)(g(s))^{j}=g_{n}(g(s))
\end{aligned}
$$

Mais além

$$
G_{2}(s)=g_{1}(g(s))=g(g(s))=g\left(g_{1}(s)\right)
$$

e analogamente

$$
g_{n+1}(s)=g\left(g_{n}(s)\right)
$$

O processo de ramificação acima vai parar tão $\operatorname{logo} P\left(X_{n}=0\right)=1$ para algum inteiro positivo $n$. A condição necessária e suficiente para isso acontecer é dada por $g^{\prime}(1)=\lambda<1$.

Vamos assumir agora que o processo de transmissão se encerra após a enésima geração, de modo que $Y_{n}=X_{0}+X_{1}+X_{2}+\ldots+X_{n}$, onde $X_{0}=1$. Definimos $G_{n}(s)$ como a FGP de $Z_{n}=X_{1}+\ldots+X_{n}$. Portanto $G_{1}(s)=g_{1}(s)=g(s)$, e a FGP de $Y_{1}$ é

$$
E\left[t_{1}^{Y}\right]=s g(s)
$$

Denotaremos essa função por $R_{1}(s)$. Uma vez que cada indivíduo infectado de $X_{1}$ começará uma nova geração, a FGP de $Z_{2}$ é

$$
G_{2}(s)=g\left(s G_{1}(s)\right)=g\left(R_{1}(s)\right)
$$

e analogamente

$$
G_{n}(s)=g\left(R_{n-1}(s)\right), \quad n=2,3,4, \ldots
$$

Assim a FGP de $Y_{n}=1+Z_{n}$ será 


$$
R_{n}(s)=s G_{n}(s)=s g\left(R_{n-1}(s)\right)
$$

Tomando o limite quando $n$ cresce temos

$$
G(s)=\lim _{n \rightarrow \infty} R_{n}(s)=s g\left[\lim _{n \rightarrow \infty} R_{n-1}(s)\right]=s g(G(s))
$$

Fazendo $G(s)=t$ na equação acima

$$
t=s g(t)
$$

Portanto, a expansão de Lagrange de $t$ como função de $s$ pode ser obtida fazendo $f(t)=t$. Isso faz com que a distribuição do número total de indivíduos infectados gerados por um único ancestral seja

$$
P\left(Y=j \mid X_{0}=1\right)=\frac{(j \lambda)^{j-1} e^{-j \lambda}}{j !}, \quad j=1,2, \ldots
$$

Essa distribuição é conhecida como a distribuição de Borel. Se $X_{0}=M$ com probabilidade 1 , então $f(t)=t^{M}$ e

$$
P\left(Y=j \mid X_{0}=M\right)=\frac{M}{(j-M) !} j^{j-M-1} \lambda^{j-M} e^{-\lambda j}, \quad j=M, M+1, \ldots
$$

que é conhecida como a distribuição de Borel-Tanner. No caso em que $X_{0}$ for uma variável aleatória com FGP $f(t)=\exp [\theta(t-1)]$, como assumimos mais cedo nessa seção, a distribuição do número total de indivíduos infectados será obtida multiplicando-se (2.46) pela Poisson

$$
P\left(X_{0}=M\right)=\frac{e^{-\theta} \theta^{M}}{M !}
$$

e somando-se sobre todos os valores de $M$. Desse modo, a distribuição do número total de indivíduos sendo infectados torna-se

$$
P(Y=j)=\frac{\theta(\theta+\lambda j)^{j-1} e^{-\theta-j \lambda}}{j !}, \quad j=0,1,2, \ldots
$$

que é o modelo da DPG com parâmetros $(\theta, \lambda)$.

\subsubsection{Processos termodinâmicos}

Considere um processo termodinâmico cujas reações acontecem entre os estados $x(=0,1,2, \ldots)$, todos na mesma fase química e onde as transições do estado $E_{k}$ só são permitidas até os estados vizinhos $E_{k}, E_{k-1} \mathrm{e}$ $E_{k+1}$. Seja $a_{k}$ a taxa de salto do estado $E_{k}$ até o estado $E_{k+1}$ e $b_{k}$ a taxa de salto do estado $E_{k}$ até o estado $E_{k-1}$. Assumimos que as taxas são as mesmas para todos os estados e que $f_{x}(t)$ denota a concentração relativa do estado $E_{x}$ em qualquer instante $t$. Pode ser facilmente demonstrado que a distribuição transiente dos estados é dada pela solução do sistema de equações diferenciais

$$
\begin{aligned}
& f_{0}^{\prime}(t)=b_{1} f_{1}(t)-a_{0} f_{0}(t) \\
& f_{x}^{\prime}(t)=b_{x+1} f_{x+1}(t)-a_{x} f_{x}(t)-\left[b_{x} f_{x}(t)-a_{x-1} f_{x-1}(t)\right]
\end{aligned}
$$


para $x=1,2,3, \ldots, \operatorname{com} f_{x}^{\prime}(t)$ a derivada de $f_{x}(t)$ com respeito a $t$.

Quando o processo termodinâmico está em equílibrio (i.e., $t \rightarrow \infty$ ) o sistema acima indica que $f_{x}(t) \rightarrow f_{x}$ e $f_{x}^{\prime}(t)=0$ e as relações do estado de equilíbrio são:

$$
\begin{aligned}
& f_{1}=\frac{a_{0}}{b_{1}} f_{0} \\
& b_{x+1} f_{x+1}=\left(a_{x}+b_{x}\right) f_{x}-a_{x-1} f_{x-1}, \quad x=1,2,3, \ldots
\end{aligned}
$$

o que resulta na distribuição

$$
f_{x}= \begin{cases}f_{0} & \text { para } x=0 \\ f_{0} \prod_{j=1}^{x} \frac{a_{j-1}}{b_{j}} & \text { para } x=1,2,3, \ldots\end{cases}
$$

Se $f_{x}$ é uma distribuição de probabilidade, sua soma sobre $x=0,1,2, \ldots$ deve ser igual a 1 - o que implica que o limite de $f_{x}$ quando $x \rightarrow \infty$ deve ser 0 (Feller [16])). A expressão acima fornece a distribuição no equilíbrio de um processo de energia cinética de primeira ordem, com taxas para frente e para trás $a_{k}$ e $b_{k}$ respectivamente. Se fizermos

$$
a_{k}=\frac{1}{(\theta+k \lambda)^{k-1}}
$$

e

$$
b_{k}=\frac{k e^{\lambda}}{(\theta+k \lambda)^{k-1}}
$$

a distribuição em equilíbrio será dada por

$$
f_{x}=\frac{\theta(\theta+x \lambda)^{x-1} e^{-x \lambda}}{x !} f_{0}
$$

Somando a expressão acima para $x=0,1,2,3, \ldots$ a hipótese de que a soma é 1 fornece $f_{0}=e^{-\theta}$. Portanto, nesse caso, $f_{x}$ será a DPG $P_{x}(\theta, \lambda)$.

\subsection{Outras propriedades da DPG}

Vimos na seção anterior alguns modelos que tem a DPG como ferramenta útil. Uma vez tendo estabelecido a importância dessa distribuição, prosseguimos com mais alguns fatos importantes da DPG.

\subsubsection{Momentos de ordem mais alta}

Para os modelos DPG com $\lambda<1$, e para o modelo restrito com $\alpha \theta<1$, todos os momentos existem. Os momentos de ordem k centrados na origem, e os centrados na média (respectivamente $\mu_{k}^{\prime}$ e $\mu_{k}$ ) são definidos da seguinte forma:

$$
\mu_{k}^{\prime}=\sum_{x=0}^{\infty} x^{k} P_{x}(\theta, \lambda) \quad \mu_{k}=\sum_{x=0}^{\infty}(x-\mu)^{k} P_{x}(\theta, \lambda)
$$


Os quatro primeiros momentos da DPG, conforme derivados por Consul e Jain [5], são dados pelas expressões seguintes. Utilizamos a notação $M=(1-\lambda)^{-1}$.

$$
\begin{aligned}
& \mu=\theta M, \quad \sigma^{2}=\mu_{2}=\theta M^{3} \\
& \mu_{3}=\theta(1+2 \lambda) M^{5}, \quad \mu_{4}=3 \theta^{2} M^{6}+\theta\left(1+8 \lambda+6 \lambda^{2}\right) M^{7}
\end{aligned}
$$

Para o cálculo dos momentos de ordem mais alta, Consul e Shenton [14]) encontraram uma fórmula de recorrência, especificamente para os momentos não-centrais (centrados na origem):

$$
(1-\lambda) \mu_{k+1}^{\prime}=\theta \mu_{k}^{\prime}+\theta \frac{\partial \mu_{k}^{\prime}}{\partial \theta}+\lambda \frac{\partial \mu_{k}^{\prime}}{\partial \lambda}, \quad k=0,1,2, \ldots
$$

A demonstração dessa equação é apresentada a seguir, e parte da utilização da função geradora de momentos da DPG.

Demonstração. Para obter a função geradora de momentos (FGM) da DPG, tomamos a sua FGP

$$
G_{x}(u)=e^{\theta(t-1)}, \quad \text { onde } t=u e^{\lambda(t-1)}
$$

Fazendo $t=e^{s}$ e $u=e^{\beta}$ na Função Geradora de Probabilidades e tomando o logaritmo da segunda expressão, obtemos

$$
\phi_{X}(\beta)=e^{\theta\left(e^{s}-1\right)}
$$

onde

$$
s=\beta+\lambda\left(e^{s}-1\right)
$$

que representa a função geradora de momentos $\phi_{X}(\beta)=E\left[e^{\beta x}\right]$.

Diferenciando (2.58) parcialmente com respeito a $\beta$ e $\lambda$, obtemos após simplificação

$$
\frac{\partial s}{\partial \beta}=\left(1-\lambda e^{s}\right)^{-1}, \quad \lambda \frac{\partial s}{\partial \lambda}=-1+\frac{1-\lambda}{1-\lambda e^{s}}
$$

A partir disso, conseguimos a seguinte equação diferencial parcial:

$$
(1-\lambda) \frac{\partial s}{\partial \beta}-\lambda \frac{\partial s}{\partial \lambda}=1
$$

Tomando agora as derivadas parciais de $(2.57)$ com respeito a $\theta, \lambda$, e $\beta$, lembrando que $\partial s / \partial \theta=0$, temos:

$$
\begin{aligned}
& \theta e^{s} \phi_{X}(\beta)=\theta \frac{\partial \phi_{X}(\beta)}{\partial \theta}+\theta \phi_{X}(\beta) \\
& \theta e^{s} \phi_{X}(\beta) \frac{\partial s}{\partial \lambda}=\frac{\partial \phi_{X}(\beta)}{\partial \lambda} \\
& \theta e^{s} \phi_{X}(\beta) \frac{\partial s}{\partial \beta}=\frac{\partial \phi_{X}(\beta)}{\partial \beta}
\end{aligned}
$$


Multiplicando (2.59) por $\theta e^{s} \phi_{X}(\beta)$ e usando o sistema acima para eliminar $\partial s / \partial \lambda$ e $\partial s / \partial \beta$, obtemos após simplificação

$$
(1-\lambda) \frac{\partial \phi_{X}(\beta)}{\partial \beta}=\theta \phi_{X}(\beta)+\theta \frac{\partial \phi_{X}(\beta)}{\partial \theta}+\lambda \frac{\partial \phi_{X}(\beta)}{\partial \lambda}
$$

Substituindo $\phi_{X}(\beta)$ por $\sum_{k=0}^{\infty} \mu_{k}^{\prime} \beta^{k} / k$ ! na equação acima, e igualando os coeficientes de $\frac{\beta^{k}}{k !}$ nos dois lados encontramos a equação de recorrência (2.56).

Os momentos centrais da DPG não restrita também podem ser expressos recursivamente, da seguinte forma:

$$
\mu_{k+1}=\frac{k \theta}{(1-\lambda)^{3}} \mu_{k-1}+\frac{1}{1-\lambda}\left[\frac{d}{d t} \mu_{k}(t)\right]_{t=1}, \quad k=1,2,3, \ldots
$$

onde $\mu_{k}(t)$ é obtido substituindo-se $\theta$ e $\lambda$ na expressão para $\mu_{k}$ por $\theta t$ e $\lambda t$, respectivamente.

Outros autores abordaram o estudo dos momentos da DPG de variadas maneiras, e derivaram outras fórmulas de recorrência. Gupta [15], por exemplo, fornece as seguintes relações:

$$
\begin{aligned}
& \mu_{k+1}=\frac{\theta}{1-\alpha \theta} \frac{d \mu_{k}}{d \theta}+k \mu_{2} \mu_{k-1}, \\
& \mu_{(k)}=\frac{\theta}{1-\alpha \theta} \frac{d \mu_{(k)}}{d \theta}+\mu_{(k)} \mu_{(1)}-k \mu_{(k)}
\end{aligned}
$$

onde $\mu_{(k)}$ é o momento fatorial de ordem $k$.

O momento fatorial $\mu_{(k)}$ de uma variável aleatória que toma apenas valores inteiros não-negativos é definido por

$$
\mu_{(k)}=\sum_{x=0}^{\infty} x^{[r]} P(X=x)
$$

onde

$$
x^{[r]}= \begin{cases}x(x-1) \ldots(x-r+1) & r=0, \ldots, x \\ 0 & r>n\end{cases}
$$

Janardan (1984) CITACAO, por sua vez, obteve os momentos não-centrais e os momentos fatoriais da seguinte maneira:

$$
\begin{aligned}
& \mu_{k}^{\prime}=\sum_{i=0}^{k} \theta^{i} e^{-\theta(1+\alpha i)} f_{(\theta)}^{(i)} \frac{\Delta^{i} \theta^{k}}{i !}, \quad k=1,2,3, \ldots \\
& \mu_{(k)}=\theta^{k} e^{-\theta(1+k \alpha)} f_{(\theta)}^{(k)}, \quad k=1,2, \ldots
\end{aligned}
$$

onde

$$
f_{(\theta)}^{(k)}=\sum_{i=0}^{\infty} \frac{(1+(i+k) \alpha)^{i+k-1} \theta^{i} e^{-i \theta \alpha}}{i !}
$$

e $\Delta^{i}$ denota a diferença para frente de ordem $i$. 


\subsubsection{Assimetria e Curtose}

Pelas equações da seção anterior, podemos deduzir com facilidade a assimetria e curtose da DPG. São elas:

$$
\gamma_{1}=\frac{1+2 \lambda}{\sqrt{\theta(1-\lambda)}}, \quad \beta_{2}=3+\frac{1+8 \lambda+6 \lambda^{2}}{\theta(1-\lambda)}
$$

Da expressão para a assimetria $\left(\gamma_{1}\right)$, notamos que a distribuição torna-se mais simétrica conforme $\theta$ cresce, e tende a 0 quando $\theta$ vai para o infinito. Além disso, para qualquer valor de $\theta$, quando $\lambda$ se aproxima de 1 a assimetria vai para o infinito. A assimetria é negativa para $\lambda<-1 / 2$.

Para a curtose, como a segunda parte da expressão é sempre positiva quando $0<\lambda<1$, a distribuição é leptocúrtica para todos os valores de $\theta$. Por outro lado, conforme $\theta$ cresce, a curtose da DPG se aproxima da distribuição normal. Conforme veremos, essa não é a única similaridade entre o modelo DPG e o Normal.

Quando $\lambda$ aproxima-se de 1, os valores de $\gamma_{1}$ e $\beta_{2}$ tornam-se grandes, indicando grande assimetria e curtose. Porém, a curva da DPG para valores assim é achatada [1], indicando que as definições usuais de assimetria e curtose não têm nessa situação sua interpretação geométrica usual.

\subsubsection{Cumulantes}

Consul e Shenton [14] fornecem uma expressão de recorrência para o cálculo dos cumulantes $L_{k}$ da DPG:

$$
(1-\lambda) L_{k+1}=\lambda \frac{\partial L_{k}}{\partial \lambda}+\theta \frac{\partial L_{k}}{\partial \theta}, \quad k=1,2,3, \ldots
$$

onde $L_{1}=\theta(1-\lambda)^{-1}$.

A demonstração pode ser encontrada em Consul [3].

Os seis primeiros cumulantes podem ser facilmente calculados a partir da equação acima, e são:

$$
\begin{aligned}
& L_{1}=\theta M, \quad L_{2}=\theta M^{3}, \quad L_{3}=\theta(1+2 \lambda) M^{5}, \\
& L_{4}=\theta\left(1+8 \lambda+6 \lambda^{2}\right) M^{7}, \quad L_{5}=\theta\left(1+22 \lambda+58 \lambda^{2}+24 \lambda^{3}\right) M^{9}, \\
& L_{6}=\theta\left(1+52 \lambda+328 \lambda^{2}+444 \lambda^{3}+120 \lambda^{4}\right) M^{11}
\end{aligned}
$$

onde $M=(1-\lambda)^{-1}$.

\subsubsection{Convergência para a normal}

Teorema 2.4.4.1. Seja $X$ uma variável com distribuição de Poisson generalizada de parâmetros $\theta$ e $\lambda$, e suponha que sua média e variância são $\mu$ e $\sigma^{2}$. Então, para todos os valores de $\lambda$, a variável aleatória $Z=\frac{X-\mu}{\sigma}$ converge em distribuição para a normal padrão, quando $\theta \rightarrow \infty$.

Demonstração. A função geradorda de cumulantes $\phi_{z}(t)$ da variável aleatória Z é dada por

$$
\psi_{z}(t)=\log \left[E\left(e^{t z}\right)\right]=-\frac{\mu t}{\sigma}+\psi_{X}\left(\frac{t}{\sigma}\right)=-\frac{\mu t}{\sigma}+\frac{L_{1}}{\sigma} t+\frac{L_{2}}{\sigma^{2}} \frac{t^{2}}{2 !}+\frac{L_{3}}{\sigma^{3}} \frac{t^{3}}{3 !}+\frac{L_{4}}{\sigma^{4}} \frac{t^{4}}{4 !}+\ldots
$$


onde $L_{r}, r=1,2,3, \ldots$ são os sucessivos cumulantes da DPG. Substituindo os valores dos cumulantes conforme aparecem em (2.71), obtemos após simplificação:

$$
\begin{aligned}
\psi_{z}(t) & =\frac{1}{2} t^{2}+\frac{1+2 \lambda}{\sqrt{\theta(1-\lambda)}} \frac{t^{3}}{3 !}+\frac{1+8 \lambda+6 \lambda^{2}}{\theta(1-\lambda)}-\frac{t^{4}}{4 !}+ \\
& +\frac{1+22 \lambda+58 \lambda^{2}+24 \lambda^{3}}{\sqrt{\theta^{3}(1-\lambda)}} \frac{t^{5}}{5 !}+\frac{1+52 \lambda+328 \lambda^{2}+444 \lambda^{3}+120 \lambda^{4}}{\theta^{2}(1-\lambda)^{2}} \frac{t^{6}}{6 !}+\ldots
\end{aligned}
$$

Tomando o limite quando $\theta \rightarrow \infty$ chegamos a:

$$
\psi_{z}(t)=\frac{t^{2}}{2}
$$

que é a função geradora de cumulantes da distribuição normal padrão (CQD).

Quando $-0.5<\lambda<0.2$, mesmo um valor de $\theta$ pequeno, como por exemplo 15 , resulta numa DPG bastante próxima da normal.

\subsubsection{Convergência para a normal inversa}

Teorema 2.4.5.1. Seja $X$ uma variável aleatória com distribuição de Poisson generalizada, de parâmetros $\theta$ e ג. Seja $\sigma^{2}$ a variância de $X$. A distribuição da variável $Y=\frac{X}{\sigma}$ converge para a normal inversa com média $c$ e variância 1 quando $\theta \rightarrow \infty$ e $\lambda \rightarrow 1$ de modo que $\theta(1-\lambda)=c^{2}$.

A demonstração desse teorema parte igualmente da função geradora de cumulantes de Y. Para detalhes, ver Consul [1].

\subsubsection{Momentos incompletos}

Quando a soma que define os momentos não centrais não ocorre de 0 até $\infty$, sendo limitada à direita ou à esquerda por $x$, chamamos os momentos resultantes de momentos incompletos. Simbolizamos os momentos incompletos à direita e à esquerda, respectivamente, por ${ }_{x} I^{k}(\theta, \lambda)$ e $I_{x}^{k}(\theta, \lambda)$, e as expressões que os definem são:

$$
{ }_{x} I^{k}(\theta, \lambda)=\sum_{j=x}^{\infty} j^{k} P_{j}(\theta, \lambda), \quad I_{x}^{k}(\theta, \lambda)=\sum_{j=0}^{x} j^{k} P_{j}(\theta, \lambda)
$$

Portanto, ${ }_{0} I^{k}(\theta, \lambda)={ }_{1} I^{k}(\theta, \lambda)$ é o k-ésimo momento não-central.

Uma equação de recorrência que relaciona os momentos incompletos é dada por:

$$
\begin{aligned}
& { }_{x} I^{k}(\theta, \lambda)=\theta Q_{x-2}(\theta+\lambda, \lambda)+\frac{\theta \lambda}{\theta+\lambda} \cdot x-1 I^{k}(\theta+\lambda, \lambda)+ \\
& +\theta \sum_{i=0}^{k=2}\left(\begin{array}{c}
k-1 \\
i+1
\end{array}\right)+\frac{\lambda}{\theta+\lambda}\left(\begin{array}{c}
k-1 \\
i
\end{array}\right) \cdot x-1 I^{i+1}(\theta+\lambda, \lambda)
\end{aligned}
$$




\subsubsection{Soma de DPGs truncadas}

Sejam $X_{i}, i=1,2, \ldots, n n$ variáveis com distribuição de Poisson generalizada, truncadas à esquerda e independentes, de parâmetros $(\theta, \lambda)$ e com função densidade

$$
P(X=x)=\frac{P_{x}(\theta, \lambda)}{F_{c-1}(\theta, \lambda)}, \quad x=c, c+1, \ldots
$$

Seja então $Y=\sum_{i=1}^{n} X_{i}$. Vamos derivar a distribuição de $Y$ para os casos onde $c=1$ e $c=2$.

1. $c=1, F_{0}(\theta, \lambda)=1-e^{-\theta}$. Temos que

$$
\left[e^{\theta} F_{0}(\theta, \lambda)\right]^{n}=\left(e^{\theta}-1\right)^{n}=\sum_{s=0}^{n}\left(\begin{array}{l}
n \\
s
\end{array}\right)(-1)^{n-s} e^{s \theta}
$$

onde $e^{s \theta}$ pode ser expandido no seguinte somatório:

$$
\left(e^{\theta}-1\right)=\sum_{s=0} n\left(\begin{array}{l}
n \\
s
\end{array}\right)(-1)^{n-s} \sum_{y=n}^{\infty} \frac{(s \theta)(s \theta+\lambda y)^{y-1} e^{-\lambda y}}{y !}
$$

Divindo os dois lados por $\left(e^{\theta}-1\right)^{n}$, obtemos a distribuição da soma de $n$ DPGs independentes truncadas no 0:

$$
P(Y=y)=f_{1}(y)=\sum_{s=1}^{n} \frac{\left(\begin{array}{l}
n \\
s
\end{array}\right)(-1)^{n-s}(s \theta)(s \theta+\lambda y)^{y-1} e^{-\lambda y}}{y !\left(e^{\theta}-1\right)^{n}}
$$

2. $c=2$. Nesse caso, temos

$$
F_{1}(\theta, \lambda)=1-e^{-\theta}-\theta e^{-\theta-\lambda}
$$

Obtemos a distribuição pelo método análogo ao anterior. Sua expressão assim resulta

$$
\begin{aligned}
P(Y=y)=f_{2}(y)= & \frac{n ! \theta^{y} e^{-\lambda y}}{y !\left(e^{\theta}-1-\theta e^{-\lambda}\right)^{n}} \sum_{r=0}^{n-1}(-1)^{r}\left(\begin{array}{c}
y \\
r
\end{array}\right) \\
& \cdot \sum_{k=0}^{y-r-1}\left[\left(\begin{array}{c}
y-r-1 \\
k
\end{array}\right)\left[\frac{\lambda(y-r)}{\theta}\right]^{k} S(y-r-k, n-r)\right]
\end{aligned}
$$

\subsubsection{Estatísticas de ordem}

Nessa seção vamos analisar a distribuição das estatísticas de ordem de uma amostra cujo tamanho é uma variável aleatória com distribuição de Poisson generalizada.

Seja $\left\{X_{i}\right\}, i=1,2,3, \ldots, N$ uma amostra aleatória de tamanho N, tomada de uma população com função densidade de probabilidade $f(x)$ e distribuição acumulada $F(x)$. Denotamos por $Y_{1}, Y_{2}, \ldots, Y_{N}$ as estatísticas de ordem e o tamanho da amostra $N$ segue uma DPG com parâmetros $(\theta, \lambda)$. 
Adicionalmente, $g_{j}(y \mid n)$ é a densidade condicional da j-ésima estatística de ordem quando $N=n$ está fixado, $h_{j}$ a densidade não-condicional de $Y_{j}$ e $h_{i, j}$ a densidade conjunta não-condicional de $Y_{i}$ e $Y_{j}$. A densidade $h_{j}$ tem a seguinte expressão:

$$
h_{j}=\frac{\left(\theta e^{-\alpha \theta}\right)^{j} F_{j}^{j-1} f_{j}}{(j-1) ! e^{\theta} Q_{j}(\theta, \alpha, \theta)} \sum_{r=0}^{\infty} \frac{(1+\alpha j+\alpha r)^{r+j-1}}{r !}\left[\theta\left(1-F_{j}\right) e^{-\alpha \theta}\right]^{r}
$$

Analogamente, a densidade conjunta de $Y_{i}$ e $Y_{j}, i<j$, é dada por

$$
\begin{aligned}
h_{i, j}= & \frac{F_{j}^{i-j}\left(F_{j}-F_{i}\right)^{j-i-1} f_{i} f_{j}\left(\theta e^{-\alpha \theta}\right)^{j}}{(i-1)^{\prime}(j-i-1) ! e^{\theta} Q_{j}(\theta, \alpha \theta)} \sum_{r=0}^{\infty} \frac{(1+\alpha j+\alpha r)^{r+j-1}}{r !} \\
& \cdot\left(1-F_{j}\right)^{r} \theta^{r} e^{-r \alpha \theta}
\end{aligned}
$$

Com isso concluí-se a revisão da Distribuição de Poisson Generalizada; propriedades da distribuição foram apresentadas, e alguns modelos que a utilizam foram descritos.

O problema que interessa a seguir é a determinação do valor de $\lambda$; mais precisamente, testar se $\lambda=0$. No capítulo seguinte, será definido um teste de hipóteses aplicável a essa situação. 


\section{Capítulo 3}

\section{FBST - Full Bayesian Significance Test}

Em Pereira e Stern [2], os autores apresentam uma nova metodologia estatística para a avaliação de hipóteses, baseada em um novo paradigma para a teoria de testes que distancia-se do pensamento frequentista (falsificacionismo de Popper) e do bayesiano clássico (teoria das decisões).

O objetivo dessa metodologia será fornecer uma medida para o valor epistêmico da hipótese; um valor de evidência que agregue informação diretamente quanto ao suporte empírico da hipótese em questão. No artigo, os autores definem um teste de hipóteses, denominado FBST (Full Bayesian Significance Test), que fornece esse valor de evidência (e-valor, analogamente ao $p$-valor) a favor da hipótese baseando-se em uma medida de probabilidade definida sobre o espaço paramétrico. Esse teste pode ser aplicado diretamente à análise de hipóteses precisas (sharp hypothesis), onde tanto os testes frequentistas quanto os bayesianos clássicos encontram problemas, sem a necessidade de recursos ad hoc, e possui diversas propriedades desejáveis, a saber:

1. Fornece uma medida simples e intuitiva para a significância da hipótese em teste: idealmente, uma medida de probabilidade diretamente definida no espaço paramétrico natural.

2. Tem uma definição intrinsicamente geométrica, e invariante (independente da parametrização específica da hipótese sob teste).

3. Fornece uma medida que seja contínua e diferenciável, sob condições apropriadas de regularidade do modelo.

4. Segue o princípio da verossimilhança: toda informação da amostra será representada pela função de verossimilhança.

5. Não necessita de nenhum procedimento artificial ad hoc, como a atribuição de uma probabilidade positiva a conjuntos de medida 0 .

6. É uma função de suporte possibilística, onde o suporte de uma disjunção lógica é o suporte máximo entre os suportes dos conjuntos disjuntos.

7. Fornece um teste consistente para hipóteses precisas (sharp hypothesis).

8. Aceita a composição de hipóteses simples em modelos mais complexos.

9. É um procedimento exato, sem necessidade de considerações assintóticas.

10. Permite a utilização de informação passada ou opiniões de especialistas, via distribuições a priori subjetivas.

O teste, tal como será definido nesse capítulo, pode também ser derivado por um argumento plenamente bayesiano a partir de uma função de perda particular, conforme demonstrado em Madruga et al [17]. 
Nesse capítulo vamos descrever o FBST, tanto teoricamente quanto no âmbito do cálculo computacional do e-valor.

\subsection{Definição do e-valor}

Seja $\theta \in \Theta \subseteq \Re^{p}$ o vetor de parâmetros de interesse, e $L(\theta \mid x)$ a função de verossimilhança para uma amostra $x$. Sob o paradigma bayesiano, a densidade a posteriori desse modelo será proporcional à verossimilhança e à distribuição a priori, da seguinte forma:

$$
p_{n}(\theta) \propto L(\theta \mid X) p_{0}(\theta)
$$

A hipótese nula $H_{0}$ é definida por restrições de igualdade e por inequações, descritas respectivamente pelas funções vetoriais $g$ e $h$, com domínio igual ao espaço paramétrico.

$$
\Theta_{H}=\{\theta \in \Theta \mid g(\theta) \leq 0 \wedge h(\theta)=0\}
$$

O interesse principal do FBST está nas hipóteses precisas - ou seja, hipóteses com pelo menos uma restrição de igualdade. Nesses casos, portanto, o espaço paramétrico sob $H_{0}$ terá menor dimensão do que o $\Theta$ original.

Para o cálculo do valor da evidência em favor de $\mathrm{H}, e v(H)$, e da evidência contra $\mathrm{H}, \overline{e v}(H)$, definimos em primeiro lugar a surpresa a posteriori, relativa a uma densidade de referência $r(\theta)$ :

$$
\begin{aligned}
& s(\theta)=\frac{p_{n}(\theta)}{r(\theta)} \\
& s^{*}=s\left(\theta^{*}\right)=\sup _{\theta \in \Theta_{H}} s(\theta), \quad \hat{s}=s(\hat{\theta})=\sup _{\theta \in \Theta} s(\theta)
\end{aligned}
$$

Essa função de surpresa, baseada na densidade de referência $r$, é utilizada na definição do teste para garantir a invariância do valor de evidência com respeito às parametrizações de $\Theta$ e $\Theta_{H}$.

Definimos, em seguida:

$$
\begin{aligned}
& T(v)=\{\theta \in \Theta \mid s(\theta) \leq v\}, \quad W(v)=\int_{T(v)} p_{n}(\theta) d \theta \\
& \bar{T}(v)=\Theta-T(v), \quad \bar{W}(v)=1-W(v)
\end{aligned}
$$

e, por fim

$$
e v(H)=W\left(s^{*}\right), \quad \overline{e v}(H)=\bar{W}\left(s^{*}\right)=1-e v(H)
$$

Portanto, o valor da evidência em favor de $\mathrm{H}$ é dado pela integral da densidade a posteriori, sobre a região do espaço paramétrico com valor de surpresa menor do que a surpresa calculada em $s^{*}$, o ponto de surpresa máxima sob $H_{0}$.

Para ilustrar as explicações, suponha que queremos testar a hipótese nula $H_{0}: \mu=0$, num modelo Gaussiano, $X \sim N\left(\mu, \sigma^{2}\right)$, com base numa amostra $\left\{x_{i}\right\}_{i=1}^{n}$. Nesse caso, temos: 


$$
\begin{aligned}
& \theta=(\mu, \sigma) \\
& \Theta=\left\{\mu, \sigma^{2} \in \Re \mid \sigma^{2}>0\right\} \\
& \Theta_{H}=\left\{\mu, \sigma^{2} \in \Re \mid \mu=0 \wedge \sigma^{2}>0\right\} \\
& p_{n}(\theta \mid X)=\prod_{i=1}^{n}\left(2 \pi \sigma^{2}\right)^{-\frac{1}{2}} \exp \left\{-\frac{\left(x_{i}-\mu\right)^{2}}{\sigma^{2}}\right\} \\
& \Omega=\Re
\end{aligned}
$$

\subsubsection{Observações sobre o FBST}

Um ponto recorrente na discussão sobre testes de hipóteses é a questão dos nuisance parameters: se uma distribuição qualquer tem mais de um parâmetro, mas a hipótese de interesse não faz alusão a todos eles, chamamos nuisance parameters aos parâmetros que não são referidos diretamente pela hipótese.

A maior parte da teoria estatística recomenda a eliminação desses parâmetros "extras", seja pela utilização da maximização da densidade com relação aos parâmetros livres, ou à sua integração. O FBST não segue esse paradigma de eliminação, e trabalha todo o tempo sobre o espaço paramétrico original, com dimensão completa.

Outra característica do FBST é a necessidade, para o cálculo do valor de evidência, de dois procedimentos numéricos: otimização (para cálculo do $s^{*}$ ) e integração (para cálculo do e-valor).

A teoria estatística clássica se serve com frequência de procedimentos de otimização (máxima verossimilhança, teste da razão de verossimilhanças, etc.), enquanto que os métodos bayesianos apoiam-se mais na integração numérica. O FBST, refletindo o fato de ser um método mais amplo, vai utilizar os dois.

Nos cálculos do presente trabalho, o método de otimização utilizado será o da biblioteca de funções em C chamada NAG (Numerical Algorithms Group- http://www.nag.com.uk) - esse é uma biblioteca comercial, com diversos métodos numéricos robustos implementados.

Para a integração numérica, utilizaremos o método de Monte Carlo, com importance sampling. Nas próximas seções fornecemos uma revisão desse método, bem como a descrição do procedimento de integração para o caso específico do FBST aplicado à Poisson generalizada.

\subsection{Integração de Monte Carlo}

O princípio básico que guia a integração numérica por Monte Carlo é bastante conhecido: suponha, por exemplo, que queremos calcular a integral $\theta=\int_{0}^{1} f(x) d x$. Geramos $n$ variáveis aleatórias $\xi_{i}, i=1,2, \ldots, n$, independentes e distribuídas uniformemente em $[0,1]$. Sabemos assim que as quantidades $f_{i}=f\left(\xi_{i}\right)$ serão variáveis aleatórias independentes com média $\theta$, e portanto estimamos a integral desejada como

$$
\theta=\frac{1}{n} \sum_{i=1}^{n} f\left(\xi_{i}\right)
$$

Esse método é o mais básico possível para o cálculo de uma integral por Monte Carlo, e seu erro padrão é dado por $\sigma / \sqrt{n}$.

Existem diversas maneiras pelas quais podemos reduzir o erro da estimativa da integral. O método que escolhemos para os cálculos desse trabalho é o de importance sampling, que definimos em seguida. 


\subsubsection{Importance sampling}

A idéia principal do importance sampling é guiar o sorteio das variáveis aleatórias, procurando aumentar a probabilidade nas regiões mais "importantes"(com respeito a $f$ ) do espaço amostral. Isso é feito pela utilização de uma distribuição de probabilidades para a importância, da seguinte maneira: seja $g(x)$ uma função tomando somente valores não-negativos, e tal que

$$
G(x)=\int_{0}^{x} g(y) d y, \quad G(1)=\int_{0}^{1} g(y) d y=1
$$

Portanto, $\mathrm{G}(\mathrm{x})$ é uma função distribuição para $0 \leq x \leq 1$. Temos então que:

$$
\theta=\int_{0}^{1} f(x) d x=\int_{0}^{1} \frac{f(x)}{g(x)} g(x) d x=\int_{0}^{1} \frac{f(x)}{g(x)} d G(x)
$$

Dado $\xi$ um número aleatório com distribuição G, temos de (3.6):

$$
E\left[\frac{f(\xi)}{g(\xi)}\right]=\theta \quad \text { e } \quad \operatorname{Var}\left[\frac{f(\xi)}{g(\xi)}\right]=\int_{0}^{1}\left(\frac{f(x)}{g(x)}-\theta\right)^{2} d G(x)
$$

O valor da estimativa para $\theta$ será, como no método mais simples acima, a média dos valores obtidos para $f(x) / g(x)$.

Para procurar diminuir o erro padrão de nossa estimativa, devemos procurar escolher a função $g$ de modo a fazer o quociente $f(x) / g(x)$ o mais homogêneo (constante) possível. Por outro lado, $g$ deve ser suficientemente simples para que possamos garantir que sua integral em $[0,1]$ seja igual a 1.

Essas condições são um tanto conflitantes, pois à medida que $g$ deve ser simples, ela deve ser o mais próxima possível de $f$, que é uma função complicada a ponto de precisar de um método numérico para a estimação de sua integral; no Capítulo 4 será indicada uma solução para esse problema, de acordo com as necessidades do presente trabalho.

\subsection{FBST para a Distribuição de Poisson Generalizada}

Consideremos para a análise da Poisson generalizada a seguinte densidade a posteriori (baseada em uma priori não-informativa):

$$
p_{n}[(\theta, \lambda) \mid X] \propto \prod_{i=1}^{n} \frac{\theta\left(\theta+x_{i} \lambda\right)^{x_{i}-1} e^{-\theta-x_{i} \lambda}}{x_{i} !}
$$

A hipótese que nos interessa nesse trabalho é $H_{0}: \lambda=0$. Ou seja, queremos testar se é necessário acrescentar um parâmetro extra à Poisson, ou se a modelagem baseada em processos estocásticos homogêneos se adequa bem aos dados.

Portanto, para essa hipótese temos:

$$
\begin{aligned}
& \Theta=\{\theta, \lambda \in \Re: \theta>0 \wedge \max (-1,-\theta / m) \leq \lambda \leq 1\} \\
& \Theta_{H}=\{\theta \in \Re: \lambda=0 \wedge \theta>0\}
\end{aligned}
$$


Vamos utilizar a densidade de referência imprópria, $r(x)=1$, e portanto a função surpresa, $s(\theta)$, reduz-se à própria posteriori. Mais ainda, como utilizaremos uma densidade a priori não informativa, a posteriori será simplesmente a função de verossimilhança.

A densidade de referência aparece nas definições do teste para garantir a invariância, e para efeito dos cálculos pouco influenciará nesse caso em particular. A distribuição a priori, por seu lado, apesar de ser uma ferramenta útil passível de ser incorporada ao teste, não será utilizada nos cálculos. Uma vez que o objetivo deste trabalho é o de analisar o desempenho do FBST em situações gerais, e compará-lo a outros testes, faremos isso assumindo informação a priori nula, normalizando as condições iniciais de cada teste.

Portanto, o cálculo de $s^{*}=\sup _{(\theta, \lambda) \in \Theta_{H}} p_{n}((\theta, \lambda) \mid x)$ é o primeiro passo para determinação do valor de evidência, e nele obtemos o valor de $\theta^{*}=\operatorname{argmax}\left(p_{n}((\theta, 0) \mid x)\right)$. A partir disso, definimos a região de integração $T\left(s^{*}\right)$ como:

$$
T\left(s^{*}\right)=\left\{(\theta, \lambda) \in \Theta: p_{n}(\theta, \lambda) \leq p_{n}\left(\theta^{*}, 0\right)\right\}
$$

As integrais que deverão ser calculadas pelo método de Monte Carlo são:

$$
\begin{aligned}
& \iint_{T\left(s^{*}\right)} p_{n}(\theta, \lambda \mid X) d \lambda d \theta=\iint_{T\left(s^{*}\right)} \prod_{i=1}^{n} \frac{\theta\left(\theta+x_{i} \lambda\right)^{x_{i}-1} e^{-\theta-x_{i} \lambda}}{x_{i} !} d \lambda d \theta \\
& \iint_{\Theta} p_{n}(\theta, \lambda \mid X) d \lambda d \theta
\end{aligned}
$$

onde, conforme vimos,

$$
T\left(s^{*}\right)=\left\{\theta, \lambda \in \Re: \theta>0, \max (-1,-\theta / m) \leq \lambda \leq 1 \wedge \prod_{i=1}^{n} \frac{\theta\left(\theta+\lambda x_{i}\right)^{x_{i}-1} e^{-\theta-\lambda x_{i}}}{x_{i} !} \leq \prod_{i=1}^{n} \frac{\theta^{* x_{i}} e^{-\theta^{*}}}{x_{i} !}\right\}
$$

de modo que

$$
e v(H)=\frac{\iint_{T\left(s^{*}\right)} p_{n}(\theta, \lambda \mid X) d \theta d \lambda}{\iint_{\Theta} p_{n}(\theta, \lambda \mid X) d \theta d \lambda}
$$

Podemos redefinir a primeira integral utilizando a função indicadora $I(\theta, \lambda)=1\left\{(\theta, \lambda) \in T\left(s^{*}\right)\right\}$, da seguinte maneira:

$$
\iint_{T\left(s^{*}\right)} p_{n}(\theta, \lambda \mid X) d \theta d \lambda=\iint_{\Theta} p_{n}(\theta, \lambda \mid X) \cdot I(\theta, \lambda) d \theta d \lambda
$$

Fazendo dessa forma, para o importance sampling precisaremos de uma função $g$ que defina uma distribuição de probabilidade sobre a região de integração $\Theta$.

Buscamos portanto uma função $G(\theta, \lambda)$ tal que

$$
\begin{aligned}
& \iint_{\Theta} G(\theta, \lambda) d \theta d \lambda=1 \\
& \Theta=\{\theta, \lambda \in \Re: \theta>0 \wedge \max (-1,-\theta / m) \leq \lambda \leq 1\}
\end{aligned}
$$


$\mathrm{O}$ valor da evidência em favor de $\mathrm{H}$ torna-se, para o método de importance sampling

$$
e v(H ; X)=\frac{\iint_{T\left(s^{*}\right)} p_{n}(\theta, \lambda \mid X) g(\theta, \lambda) d \theta d \lambda}{\iint_{\Theta} p_{n}(\theta, \lambda \mid X) g(\theta, \lambda) d \theta d \lambda}
$$

Uma vez encontrada a função $g$, o quociente acima pode ser estimado por

$$
e v(H ; X)=\frac{\sum_{i=1}^{m} Z_{g}^{*}\left(\theta_{i, \diamond}, \lambda_{i, \diamond} ; X\right)}{\sum_{i=1}^{m} Z_{g}\left(\theta_{i, \diamond}, \lambda_{i, \diamond} ; X\right)}
$$

onde

$$
\begin{aligned}
& Z_{g}^{*}\left(\theta_{i, \diamond}, \lambda_{i, \diamond} ; X\right)=I\left(\theta_{i, \diamond}, \lambda_{i, \diamond}\right) Z_{g}\left(\theta_{i, \diamond}, \lambda_{i, \diamond} ; X\right) \\
& Z_{g}\left(\theta_{i, \diamond}, \lambda_{i, \diamond} ; X\right)=\frac{p_{n}\left(\theta_{i, \diamond}, \lambda_{i, \diamond} \mid X\right)}{g\left(\theta_{i, \diamond}, \lambda_{i, \diamond}\right)}
\end{aligned}
$$

e $\left(\theta_{i, \diamond}, \lambda_{i, \diamond}\right), i=1,2, \ldots, m$ são valores gerados aleatoriamente a partir da distribuição $g$. 


\section{Capítulo 4}

\section{Geração de variáveis pseudo-aleatórias}

O objetivo principal desse trabalho é o de verificar a eficiência do teste FBST para a hipótese $\lambda=0$, na Distribuição de Poisson Generalizada. Para poder mensurar essa eficiência em diversas situações, precisaremos de amostras da DPG com parâmetros variados, que obteremos através da simulação de números pseudo-aleatórios dessa distribuição.

Além disso, para a integração de Monte Carlo com importance sampling, será necessária a geração de números pseudo-aleatórios de acordo com a distribuição escolhida. Nesse capítulo, essa distribuição será definida, e um método de geração será apresentado.

O mecanismo gerador de números pseudo-aleatórios em $[0,1)$ utilizado, base para todas as simulações, foi o Mersenne Twister [24]. O código-fonte, incluído no Apêndice B, é disponibilizado pelos próprios autores do artigo original e pode ser encontrado na internet.

\subsection{Gerando números da DPG}

Existem muitos métodos de geração de números pseudo-aleatórios. No caso particular da DPG, em Consul e Famoye [19] são definidos alguns métodos específicos; no entanto, a maior parte desses métodos funciona apenas para regiões específicas do espaço paramétrico. Apenas um, o método da inversão, funciona sem restrições. Por conta disso, esse foi o algoritmo escolhido para esse trabalho.

O raciocínio subjacente ao método é bastante simples, e é apresentado a seguir.

Método da inversão Seja $X$ uma variável aleatória Lagrangiana com função distribuição e função distribuição acumulada, respectivamente:

$$
\begin{aligned}
& P\left(X=x_{j}\right)=P_{x j}, \quad x_{j} \in T \\
& P\left(X \leq x_{j}\right)=f\left(x_{j}\right)=\sum_{i \leq x_{j}} P_{i}
\end{aligned}
$$

com $T$ um subconjunto dos inteiros não-negativos. Se $U$ é uma variável uniforme em $(0,1)$, temos

$$
P\left(F\left(x_{j}-1\right)<U \leq F\left(x_{j}\right)\right)=\int_{F\left(x_{j}-1\right)}^{F\left(x_{j}\right)} d u=F\left(x_{j}\right)-F\left(x_{j}-1\right)=P_{x j}
$$


Uma variável Lagrangiana é obtida fazendo $x=x_{j}$ se

$$
F\left(x_{j}-1\right)<U \leq F\left(x_{j}\right)
$$

O método de inversão funciona, portanto, a partir da simulação de uma variável aleatória uniforme em $(0,1)$ e sua subsequente transformação.

A distribuição de Poisson generalizada, conforme apresentada aqui, é uma distribuição Lagrangiana [19], e portanto o método acima será o escolhido para a geração das amostras aleatórias da DPG. O pseudo-código para a implementação do método, de acordo com Famoye [20] é:

Método da Inversão para a DPG $\omega=e^{-\lambda}$

- 1. $X \leftarrow 0$

- 2. $S \leftarrow e^{-\theta}$ e $P \leftarrow S$

- 3. Gerar $U$ da Uniforme $(0,1)$

- 4. Enquanto $U>S$ :

$$
\begin{aligned}
& X \leftarrow X+1 \\
& c \leftarrow \theta-\lambda+\lambda X \\
& P \leftarrow \omega c(1+\lambda / c)^{X-1} P / X \\
& S \leftarrow S+P
\end{aligned}
$$

- 5. Retorna $X$

O código $\mathrm{C}++$ que implementa o método está incluído no Apêndice B.

\subsection{Distribuição para o Importance sampling}

Conforme vimos no capítulo anterior, para a implementação do método de importante sampling na integração de Monte Carlo, será necessária uma distribuição de probabilidades sobre o espaço paramétrico da DPG.

Recordando os capítulos anteriores, esse espaço paramétrico é definido por:

$$
\Theta=\{\theta, \lambda \in \Re: \theta>0 \wedge \max (-1,-\theta / m) \leq \lambda \leq 1\}
$$

Em primeiro lugar, a definição do espaço paramétrico já impõe algumas dificuldades na definição de uma distribuição única, especialmente por conta da dependência que há entre os valores dos parâmetros (por conta da constante $m)$ :

$$
m \text { é o maior inteiro tal que } \theta+m \lambda>0
$$

Por conta disso, encontrar uma distribuição diretamente definida sobre esse espaço se torna muito custoso. Ao invés de uma única distribuição, portanto, serão utilizadas duas distribuições distintas e conhecidas, com suporte coincidente com o de cada um dos parâmetros da DPG. Para construir o vetor conjunto a partir das distribuições marginais, serão utilizadas variáveis de Bernoulli para garantir que as condições (4.5) e (4.6) acima estejam satisfeitas. Serão utilizadas assim duas distribuições: uma definida na reta positiva, para sorteio do parâmetro $\theta$, e outra definida em $[-1,1]$ para $\lambda$. 
As distribuições escolhidas serão a Beta, em $[0,1]$, para o $\lambda$, com a utilização de uma variável de Bernoulli para determinar o sinal de $\lambda$, e a distribuição de Poisson para o $\theta$, truncada em $-m \lambda$ caso $\lambda<0$.

O algoritmo para simulação do vetor conjunto terá os passos:

1. Sortear $\lambda$ da distribuição Beta em $[0,1]$.

2. Sortear $u$ da Uniforme em $[0,1]$.

3. Se $u<0.5$, fazer $\lambda=-\lambda$.

4. Sortear $\theta$ da Poisson em $[0, \infty]$.

5. Se $\lambda<0$ e $\theta<-m \lambda$, retorna ao passo anterior.

O Apêndice B contém os códigos-fonte em $\mathrm{C}++$ que implementam essa distribuição para o importance sampling.

Em seguida, amostras geradas a partir desses algoritmos são apresentadas.

\subsection{Amostras}

\subsubsection{DPG}

O algoritmo definido nesse capítulo pode (e será) usado para geração de valores da DPG com diversas combinações de parâmetros. Em primeiro lugar, como o objetivo principal do trabalho é testar $H_{0}: \lambda=0$, cabe aqui observar os efeitos desse parâmetro na distribuição das amostras geradas.

Vemos, por exemplo, na Figura 4.1 os histogramas de amostras de tamanho 100, para $\theta=50$, e $\lambda \in\{-0.9,0,0.9\}$. 


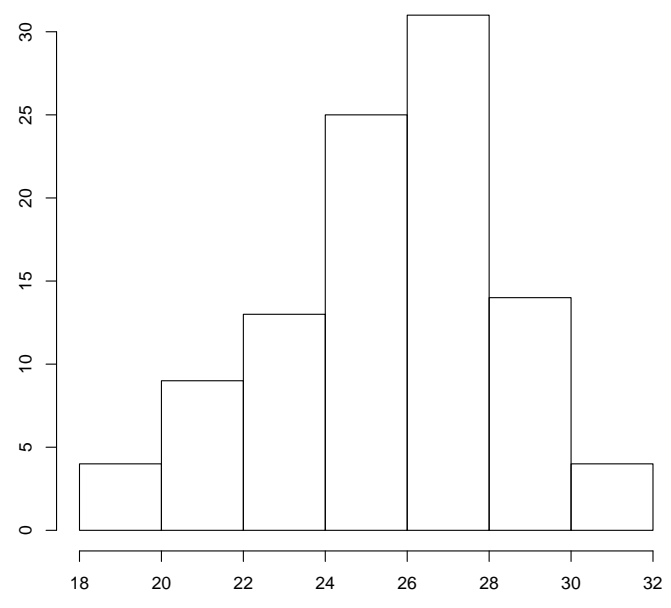

(a) $\lambda=-0.9$

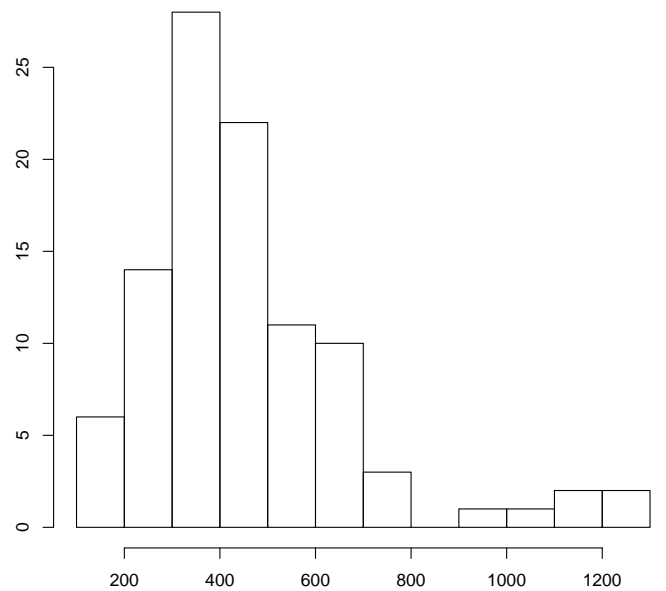

(b) $\lambda=0.9$

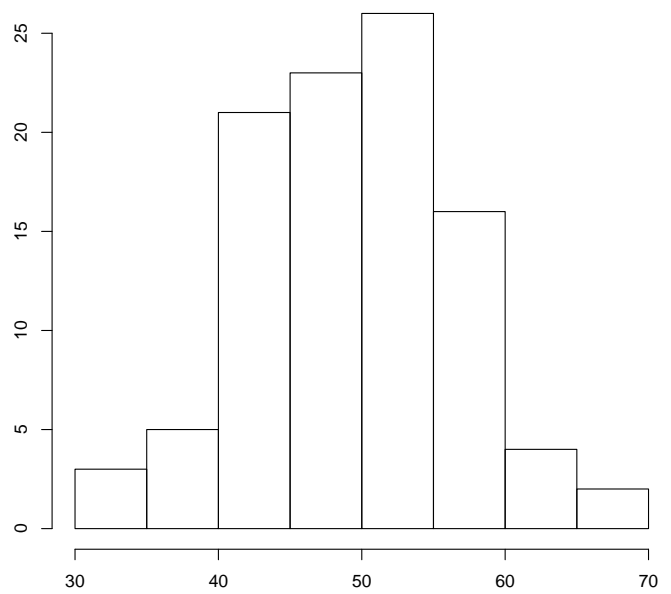

(c) $\lambda=0$

Figura 4.1: Histogramas para $\theta=50$ 
Em concordância com o que foi derivado no Capítulo 1, nota-se um aumento da assimetria quando $\lambda$ se distancia de 0 . Em particular, para $\lambda<0$ a assimetria cresce à direita, e o oposto ocorre quando $\lambda>0$. Por outro lado, os valores gerados são bem menores quando o parâmetro extra toma valores negativos; o valor de $\lambda$ retarda o número de ocorrências do processo quando é negativo, e o acelera quando positivo.

Quando $\lambda$ varia para um valor menor de $\theta$ (Figura 4.2), nota-se o mesmo comportamento; porém, a taxa de ocorrência do processo é muito menor, como era de se esperar. Para $\theta=5$ e $\lambda=0.9$, a curva se concentra fortemente no intervalo [0,100], e ocorrem valores extremos com probabilidade mais alta (i.e., ocorre um aumento de densidade nas caudas da distribuição).



(a) $\lambda=-0.9$

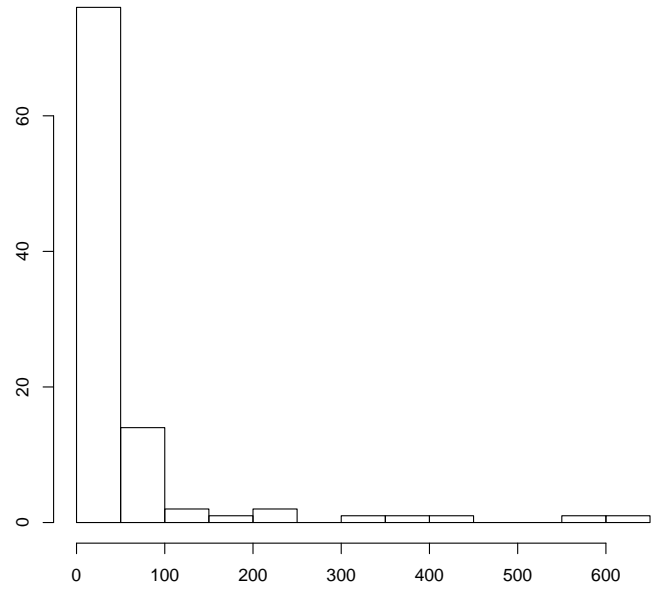

(b) $\lambda=0.9$

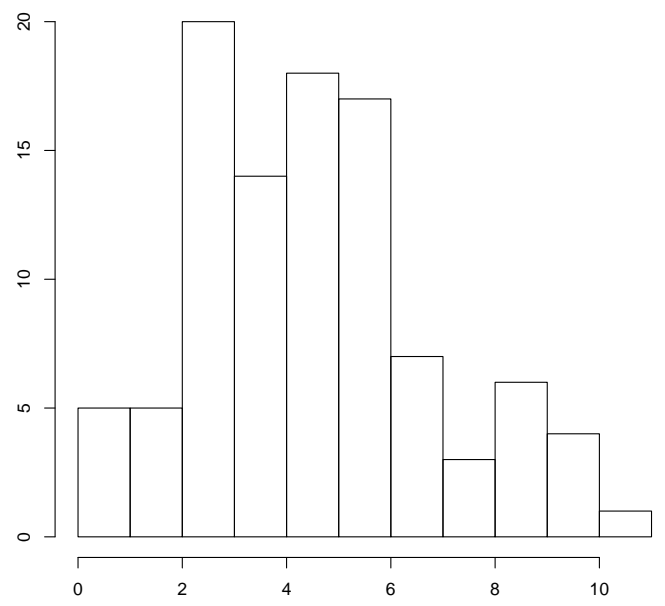

(c) $\lambda=0$

Figura 4.2: Histogramas para $\theta=5$ 


\subsubsection{Importance sampling}

O algoritmo definido para utilização no Importance sampling tem como principal objetivo preencher o espaço paramétrico, com maior frequência nas áreas de maior concentração da função de verossimilhança, que deve ser integrada.

A escolha dos parâmetros das distribuições utilizadas pelo algoritmo ( $\alpha$ e $\beta$ para a Beta, $\mu$ para a Exponencial) é importante para minimizar o erro da integral; o comportamento do algoritmo para algumas combinações de parâmetros aparece na Figura 4.3.

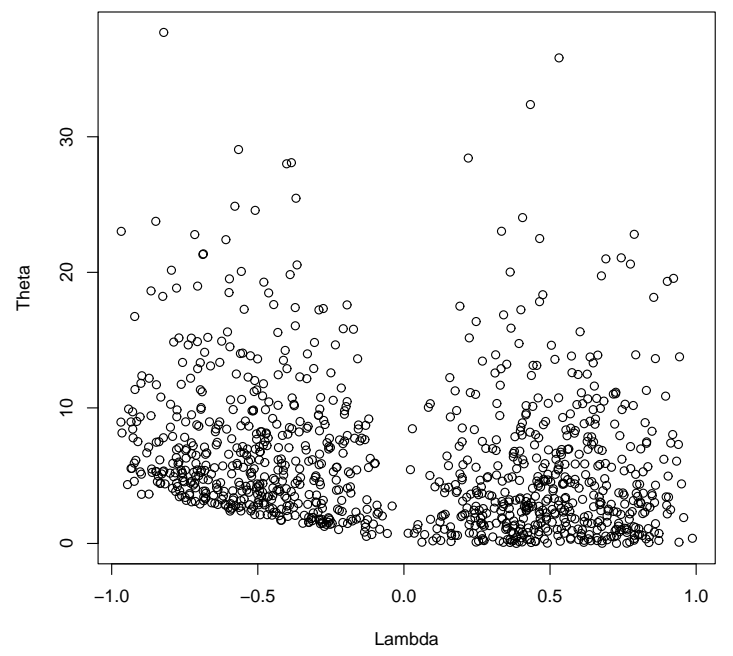

(a) $\alpha=2, \beta=2, \mu=0.2$

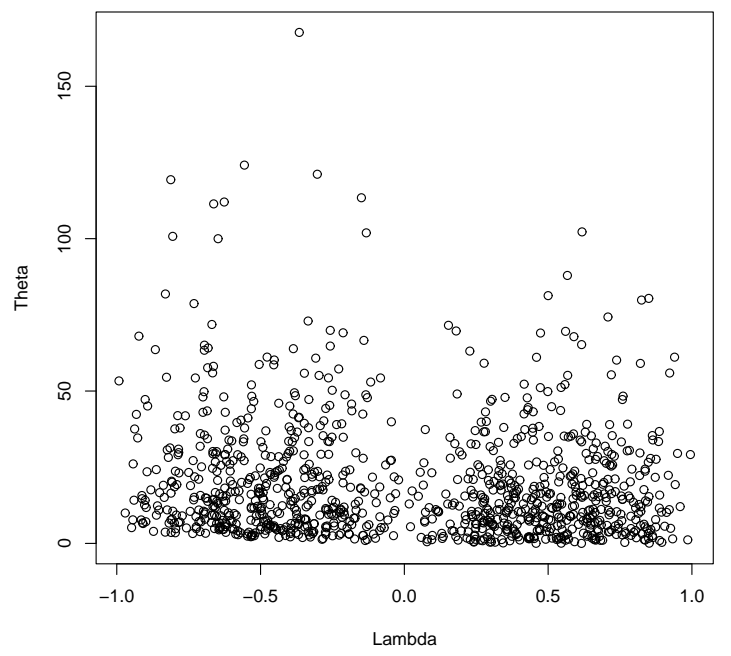

(b) $\alpha=2, \beta=2, \mu=0.05$

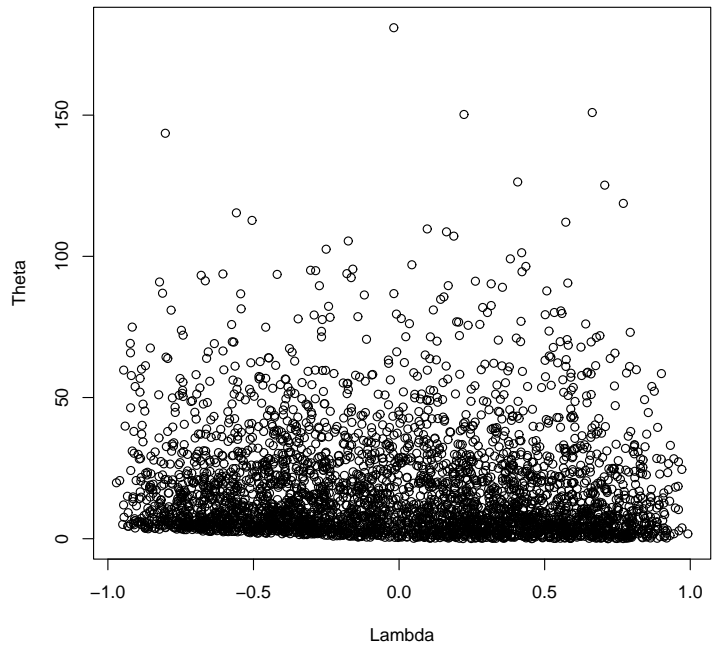

(c) $\alpha=2, \beta=1, \mu=0.05$

Figura 4.3: Histogramas para $\theta=5$ 
Vê-se duas características importantes da distribuição; em primeiro lugar, se a variância da Beta for pequena, surge uma lacuna em torno do 0. Como a escolha do sinal da Beta se dá através de uma variável de Bernoulli, é necessária uma Beta de variância maior para preencher o espaço mais próximo a esse valor.

A distribuição exponencial, por sua vez, terá que ter seu parâmetros $(\mu=1 / E[X])$ adequado à moda da verossimilhança. Um modo de conseguir isso é fazer $\mu=1 / \bar{x}$.

Nesse ponto, já foram definidas todas as ferramentas necessárias para o cálculo do FBST. No próximo capítulo, outros testes presentes na literatura são apresentados, e seu desempenho é comparado ao do FBST para o problema do parâmetro extra na DPG. 


\section{Capítulo 5}

\section{Testes e Resultados}

Uma vez definidas as técnicas a serem aplicadas para implementação do FBST, vamos nesse capítulo indicar e definir os testes já presentes na literatura, cujos resultados poderão ser comparados aos nossos. Em seguida, alguns comentários serão incluídos sobre a implementação computacional dos testes.

\subsection{Teste de Consul-Famoye}

Em Consul e Famoye (1990) CITACAO, os autores derivam um teste para $H_{0}: \lambda=\lambda_{0}<0.5$ contra $H_{1}: \lambda>\lambda_{0}$ baseando-se no seguinte resultado:

Teorema 1. Seja X uma variável aleatória com distribuição de Poisson generalizada de parâmetros $\theta$ e $\lambda$. Então, se $\lambda<0.5$, a variável

$$
z=\frac{X-\mu}{\sigma}
$$

converge em distribuição para uma variável Notmal padrão quando $\theta$ cresce.

Aqui, $\mu=E(X)$ e $\sigma=\sqrt{\operatorname{var}(X)}$. A demonstração do teorema pode ser encontrada em Consul e Shenton [12]. A partir da estatística de teste $\bar{X}=\frac{1}{n} \sum X_{i}$, constrói-se a região crítica C:

$$
C=\theta_{0}\left(1-\lambda_{0}\right)^{-1}+z_{\alpha} \sqrt{\frac{\theta_{0}\left(1-\lambda_{0}\right)^{-3}}{n}}
$$

Para a nossa hipótese, $\lambda=\lambda_{0}=0<0.5$ :

$\operatorname{com} \theta_{0}$ conhecido.

$$
C=\theta_{0}+z_{\alpha} \sqrt{\frac{\theta_{0}}{n}}
$$

\subsection{Teste de Razão de Verossimilhanças Generalizado (TRVG)}

O teste de razão de verossimilhanças generalizado [21] é amplamente conhecido na prática estatística. Baseia-se na teoria frequentista de testes de hipóteses, e utiliza como estatística o valor 


$$
q=-2 \ln \frac{\sup _{(\lambda, \theta) \in \Theta_{H}} L((\lambda, \theta), X)}{\sup _{(\lambda, \theta) \in \Theta} L((\lambda, \theta), X)}
$$

onde $q$ tem distribuição (assintótica) qui-quadrada, com número de graus de liberdade dado pela diferença entre o número de parâmetros não-especificados em $\Theta$ e $\Theta_{H}$ (no caso desse estudo, 1 grau de liberdade apenas).

No caso da DPG, os espaços paramétricos irrestrito e sob $H_{0}$ têm a forma dada em (3.3). Vamos calcular a estatística $\chi^{2}$ por otimização numérica das funções de log-verossimilhança restrita e irrestrita.

\subsection{Implementação dos testes}

Assim como o FBST, os testes de Consul-Famoye e Razão de Verossimilhanças foram implementados diretamente em $\mathrm{C}++$. A escolha da linguagem se deve, primordialmente, à possibilidade de utilização do paradigma da orientação a objetos, cuja principal vantagem é a facilidade de modelagem dos problemas e de reutilização do código.

Será implementada uma classe para a Distribuição de Poisson Generalizada ( $c l D P G)$, e classes para os testes de hipóteses (clFBST, clCF e clRVG).

Para as classes que implementam os testes, havia uma escolha a ser feita: como muitas das funções numéricas seriam utilizadas no cálculo dos três testes, poderia ser definida uma classe para conter essas funções gerais. Além disso, uma modelagem orientada a objetos poderia passar pela definição de uma classe abstrata representando um teste de hipóteses geral. Porém, a opção adotada foi a de manter cada classe com o máximo de auto-suficiência.

Algoritmos para otimização e integração numéricas serão necessários para o cálculo dos testes, mas apenas a integração será implementada neste trabalho. Conforme indicado mais acima, a biblioteca NAG - Numerical Algotihms Group fornecerá o algoritmo de otimização utilizado no código.

Há também uma classe específica para o gerador de números pseudo-aleatórios (clMersenneTwister). O código completo de todas as classes está no Apêndice B.

\subsection{Espaço paramétrico}

Os testes de Consul-Famoye e de Razão de Verossimilhanças dependem explicitamente de considerações assintóticas, ao contrário do FBST. Cabe, portanto, comparar os três testes em diferentes tamanhos de amostra. Da mesma forma, é importante observar as performances dos testes para diferentes valores de $\theta$ (especialmente para o teste de Consul-Famoye, cuja convergência ocorre quando $\theta \rightarrow \infty$ ).

Simularemos, portanto, amostras de tamanho 20, 40, 60 e 100 da DPG, com a dupla de parâmetros $(\theta, \lambda)$ assumindo todos os valores em $[1,2,3,4,5,6,7,8,9,10,25,50] \times[-0.9,-0.8, \ldots, 0, \ldots, 0.8,0.9]$.

A hipótese nula a ser testada será, sempre, $H_{0}: \lambda=0$.

Para mitigar os efeitos de erros numéricos e abrandar as flutuações mais extremas das amostras, para cada valor de $N$ os procedimentos foram repetidos diversas vezes, e ao final foi obtida a média dos valores de evidência para cada teste, em cada tamanho de amostra, e para cada combinação de valores dos parâmetros (os procedimentos foram repetidos 60 vezes para cada tamanho de amostra).

Isso posto, apresentamos a seguir os resultados das simulações. 


\subsection{Resultados}

Ao longo dessa seção, e no restante desse trabalho, denominaremos e-valor, $p$-valor e $q$-valor aos valores de evidência obtidos do FBST, do teste de Consul-Famoye, e o teste de razão de verossimilhanças generalizado, respectivamente.

Testar se $\lambda=0$ implica, em última análise, testar se um parâmetro extra é necessário para a modelagem dos dados (i.e., se os processos estocásticos geradores dos dados são homogêneos), ou se apenas $\theta$ é suficiente para a estimação do modelo probabil'sitico. Esse parâmetro extra induz um desvio na variância da distribuição, que no caso $\lambda=0$ não ocorre (nesse caso, $E[X]=\operatorname{Var}[X]$ ).

O comportamento da média e da variabilidade (representada pelo coeficiente de variação) com relação aos parâmetros da DPG pode ser visualizado na Figura 5.1. Nota-se que, como era de se esperar, os impactos mais sensíveis surgem para valores extremos de $\lambda$; o comportamento dos testes nessas regiões do espaço paramétrico deve se mostrar mais errático do que nas proximidades de $\lambda=0$.

Os gráficos apresentandos nesse capítulo foram feitos com a utilização dos softwares Mathematica, versão 6.0 (para os gráficos de superfície em três dimensões), e $\mathbf{R}$, versão 2.3.1.



(a) Esperança

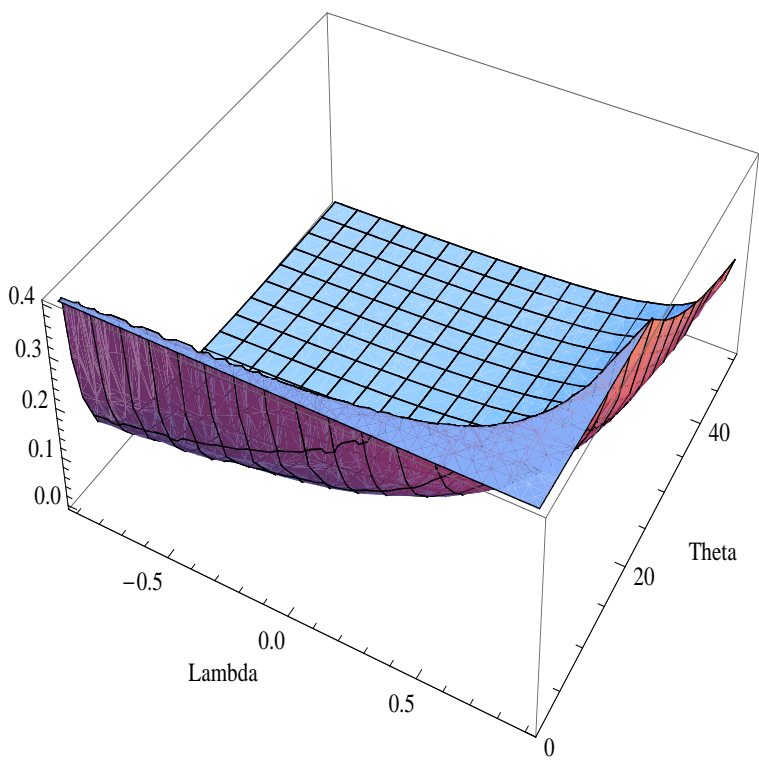

(b) Coeficiente de variação

Figura 5.1: Esperança e coeficiente de variação para a DPG 
Feitas essas considerações, a próxima seção inicia a apresentação dos resultados propriamente ditos.

\subsubsection{Valores de evidência sobre o espaço paramétrico}

Para cada teste em análise, é possível plotar a superfície dos valores de evidência, sobre os valores de $\theta$ e $\lambda$; essa primeira análise permite observar de modo mais geral o comportamento de cada medida.

Os gráficos 5.2 a 5.5 apresentam essas superfícies, e os dados completos, utilizados para a geração das figuras, encontram-se no Apêndice A.

Alguns pontos são imediatamente dignos de atenção. O teste de Consul-Famoye, por exemplo, por admitir a hipótese alternativa $H_{1}: \lambda>0$, atribui p-valor 1 para todos os casos de $\lambda$ negativo; na prática, portanto, esse teste seria mais adequado à hipótese $H_{0}: \lambda \leq 0$. O teste baseia-se em $\theta$ conhecido, e portanto seu desempenho é bom levando-se em conta a deficiência na definição de $H_{0}$ e $H_{1}$.

O FBST (por não depender de definições de hipótese alternativa) e o TRVG (onde $H_{1}$ é $\lambda \neq 0$ ) apresentam resultados mais robustos sempre que $\lambda$ se distancia de $H_{0}$, seja para a esquerda seja para a direita.

Nota-se também que, conforme aumenta o tamanho da amostra, o q-valor atinge o máximo para regiões de área crescente em torno de $\lambda=\lambda_{0}$ (máximo esse que não chega a 1 em nenhum caso). Esse tipo de comportamento em testes frequentistas já foi anotado, por exemplo, por Stern [23]. A superfície do e-valor, por sua vez, se aproxima da situação ideal (com e-valor 1 para $\lambda=0$, e indo rapidamente para 0 conforme $\lambda$ se distancia desse valor).

Outra característica da superfície do q-valor, já discutida na literatura [22], é a forma do cume da superfície ao redor de $\lambda_{0}$. Nas regiões muito próximas desse valor, a superfície possui inclinação alta, beirando a não-diferenciabilidade. 


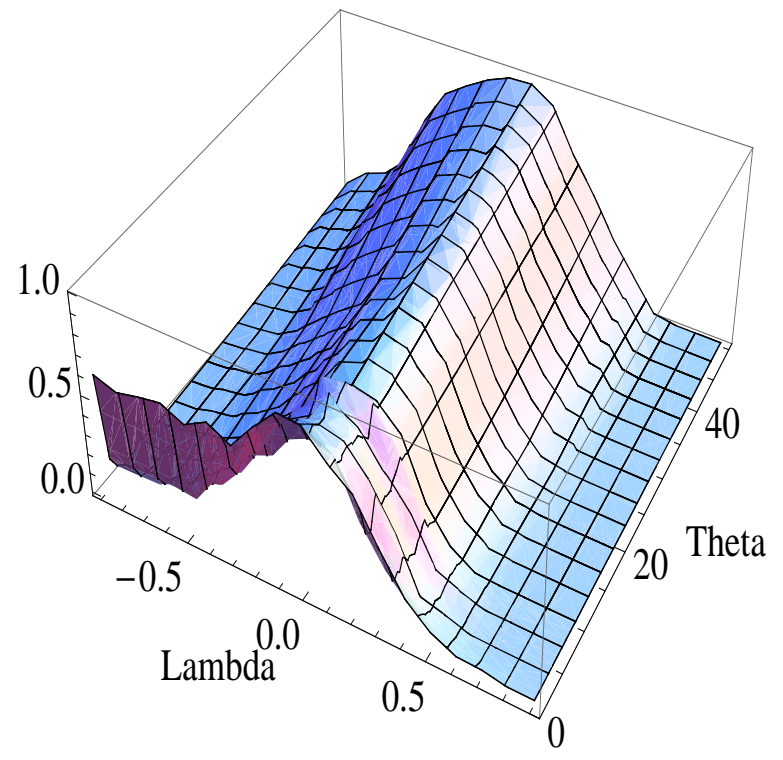

(a) e-valor

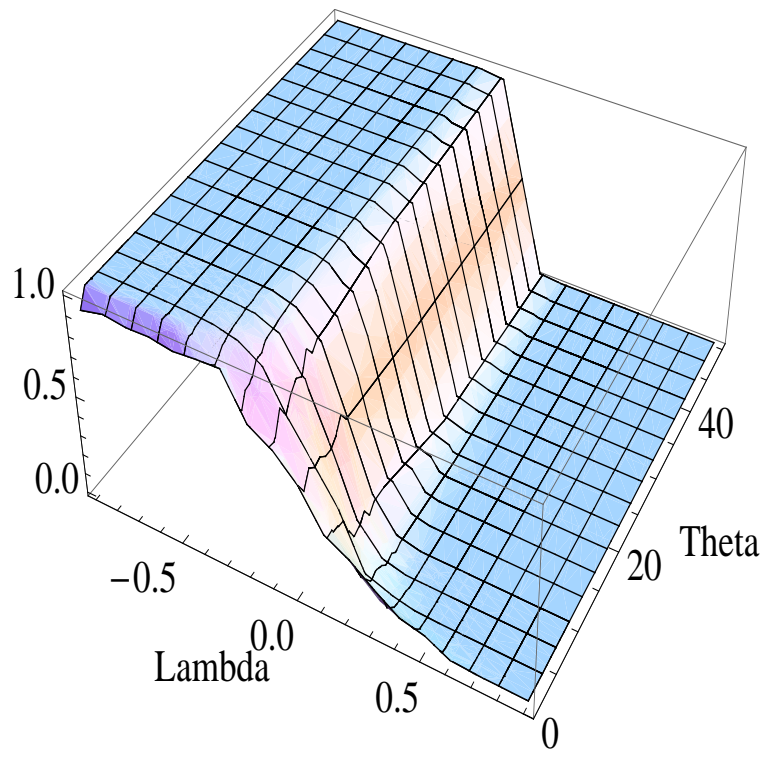

(b) p-valor

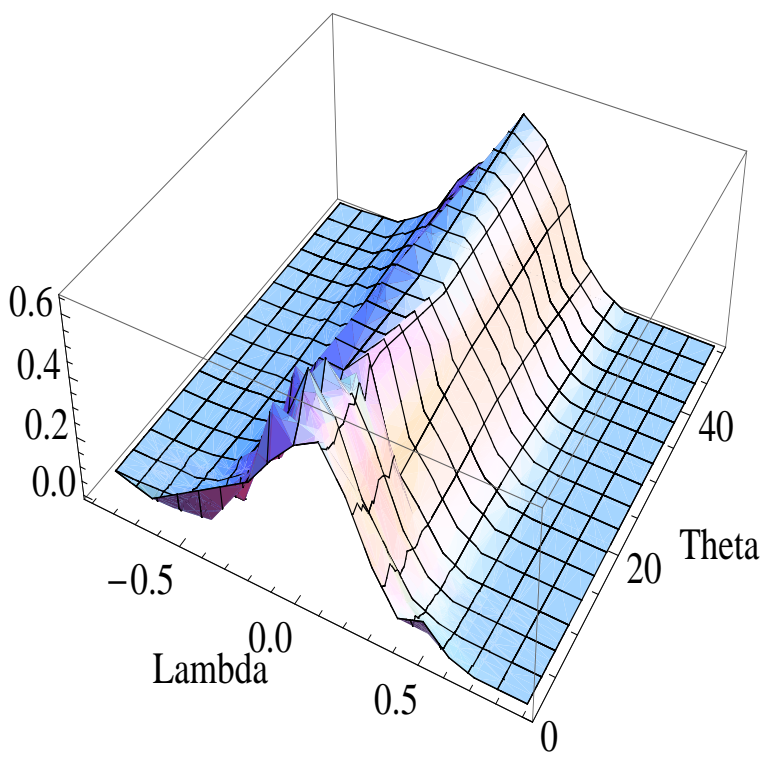

(c) q-valor

Figura 5.2: Superfícies para $N=20$ 


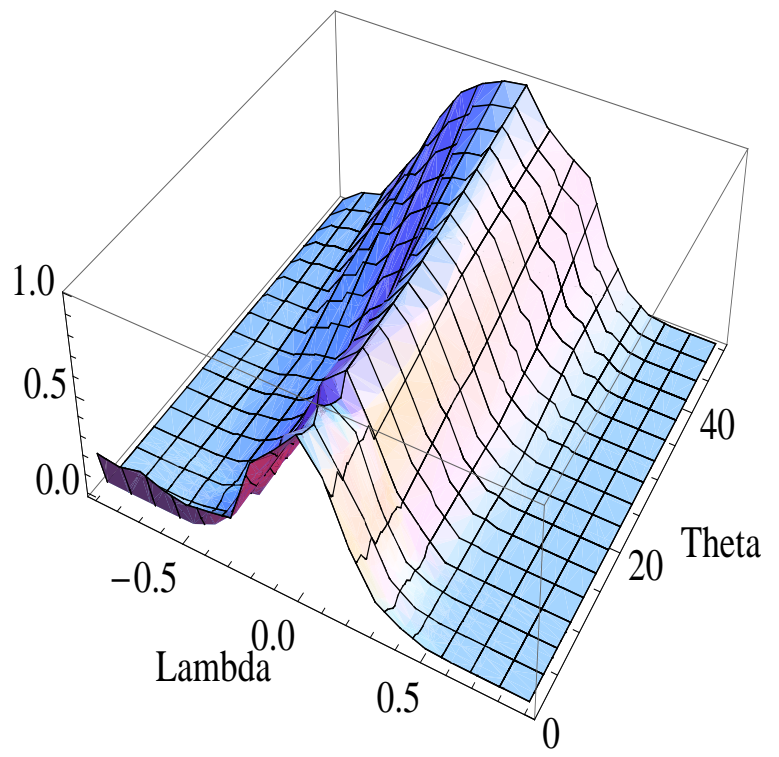

(a) e-valor



(b) p-valor

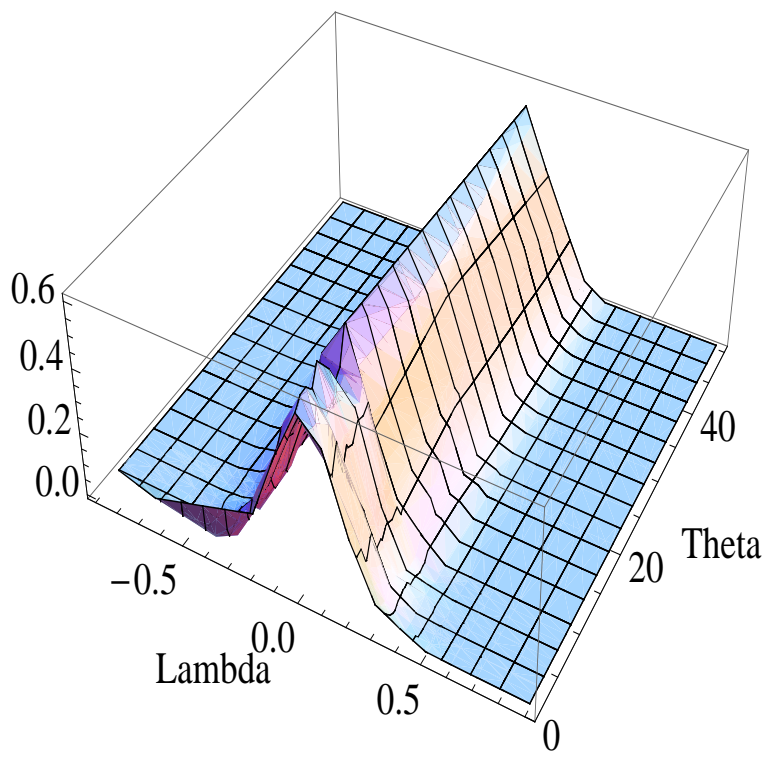

(c) q-valor

Figura 5.3: Superfícies para $N=40$ 


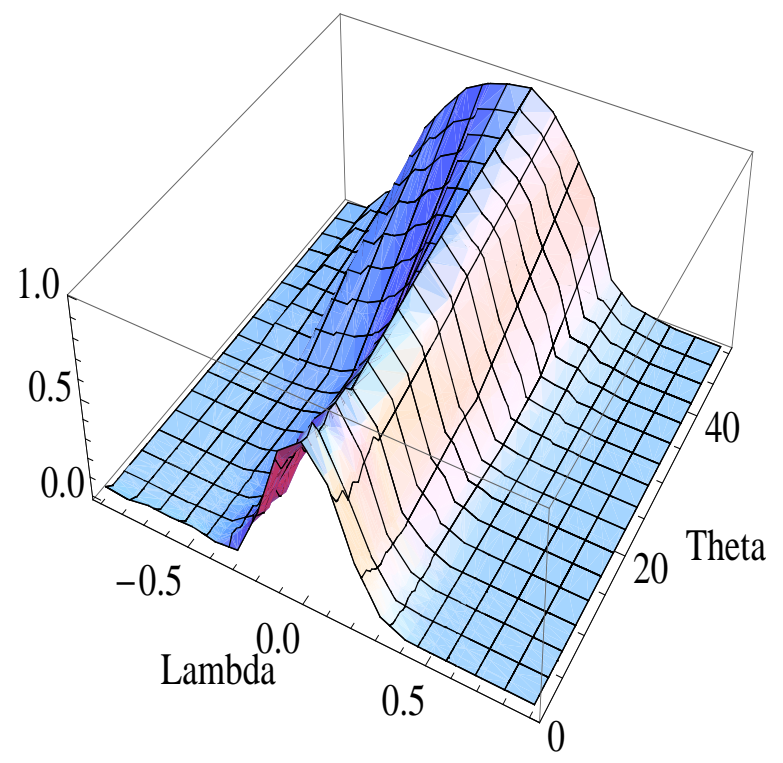

(a) e-valor

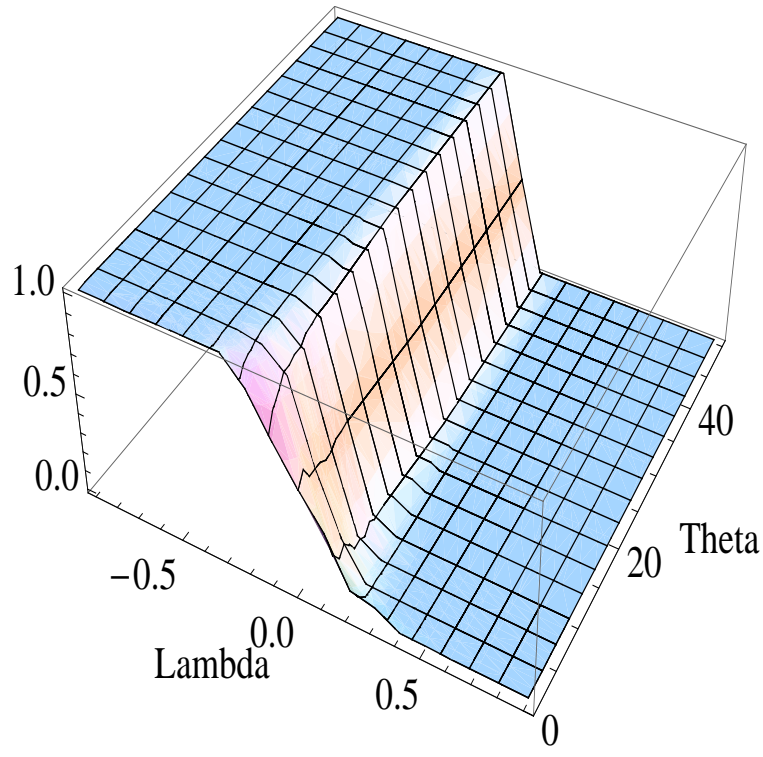

(b) p-valor



(c) q-valor

Figura 5.4: Superfícies para N $=60$ 


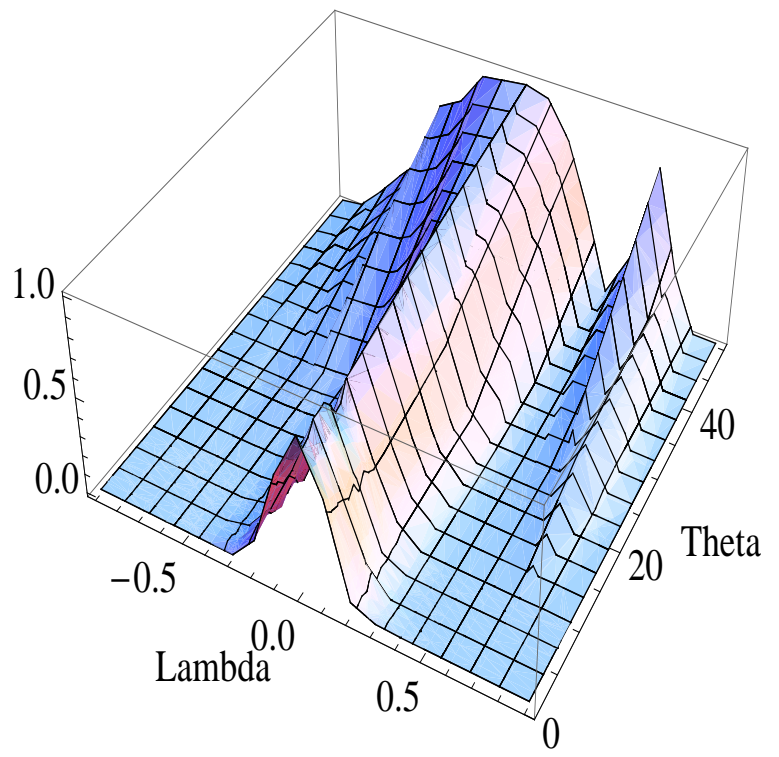

(a) e-valor

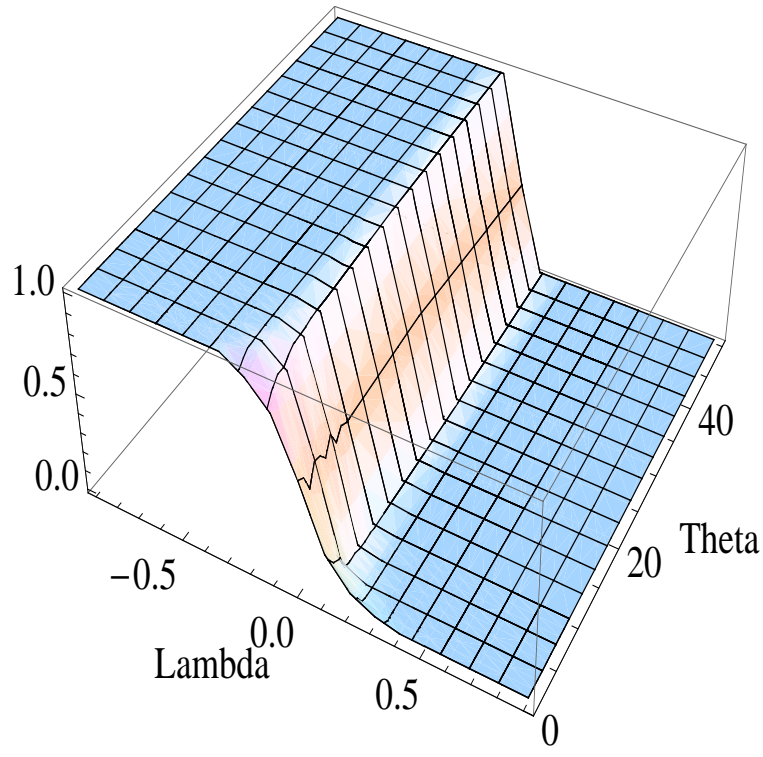

(b) p-valor

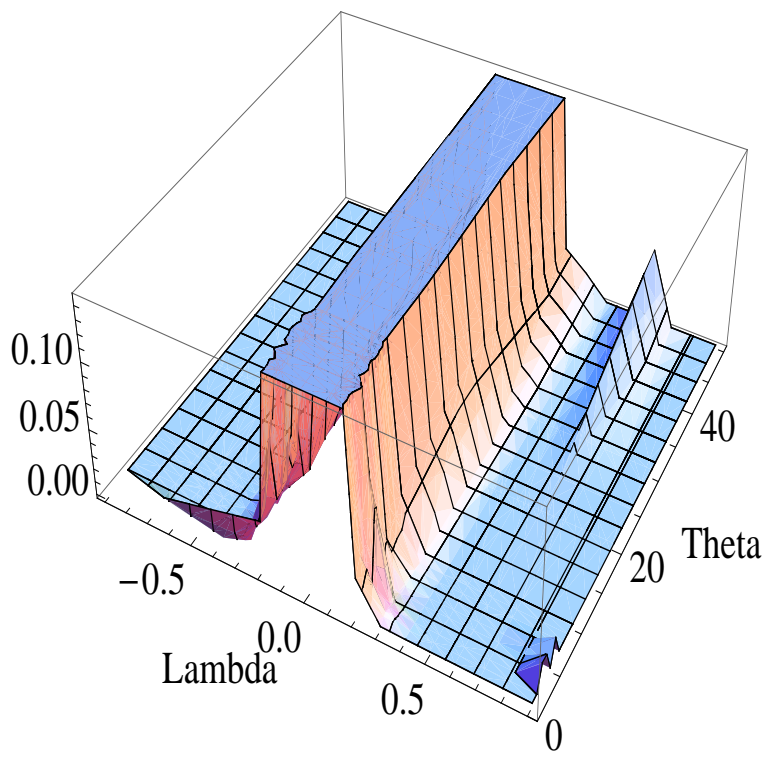

(c) q-valor

Figura 5.5: Superfícies para $\mathrm{N}=100$ 


\subsubsection{Desempenho quando $\lambda=\lambda_{0}$}

Nessa seção será comparada a performance dos três testes quando $H_{0}$ é verdadeira(i.e., quando $\left.\lambda=0\right)($ Figura $5.6)$.

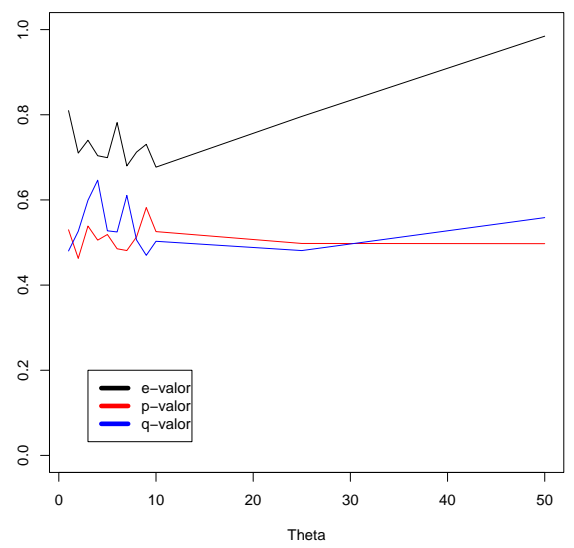

(a) $\mathrm{N}=20$

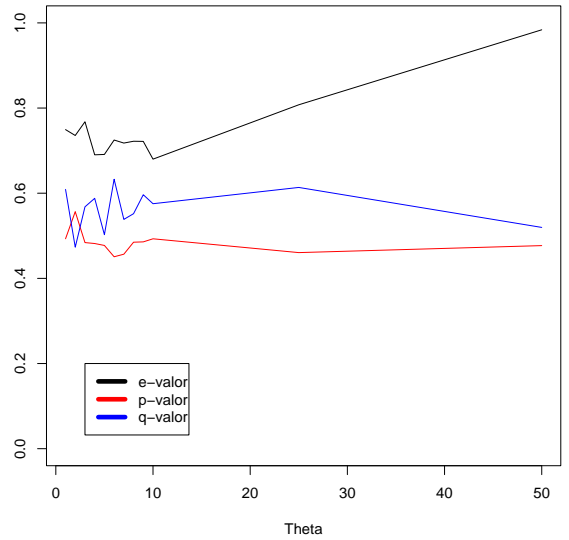

(c) $\mathrm{N}=60$

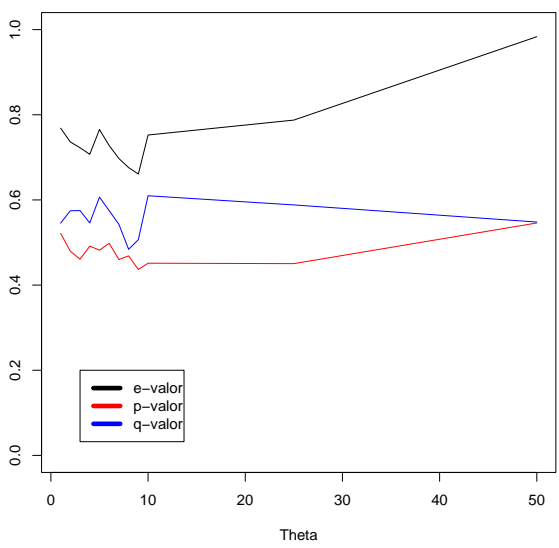

(b) $\mathrm{N}=40$

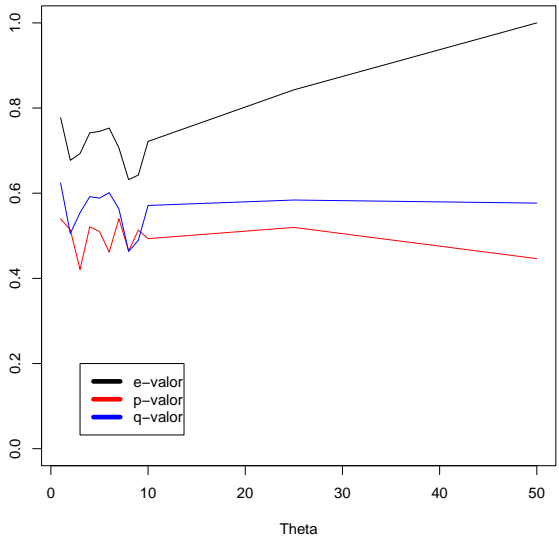

(d) $\mathrm{N}=100$

Figura 5.6: Valores de evidência em favor da hipótese para H0 verdadeira 
Os valores utilizados para construção do gráfico estão nas tabelas 5.1 e 5.2, abaixo.

\begin{tabular}{c|c|c|c|c|c|c} 
& \multicolumn{3}{|c|}{$N=20$} & \multicolumn{3}{c}{$N=40$} \\
\hline \hline$\theta$ & e-valor & p-valor & q-valor & e-valor & p-valor & q-valor \\
\hline \hline 1 & 0.809737 & 0.529861 & 0.480212 & 0.768598 & 0.521509 & 0.545408 \\
2 & 0.710029 & 0.462789 & 0.526418 & 0.736164 & 0.479469 & 0.574541 \\
3 & 0.74016 & 0.538647 & 0.599218 & 0.722571 & 0.46082 & 0.575029 \\
4 & 0.703841 & 0.505537 & 0.646376 & 0.707217 & 0.491438 & 0.5459 \\
5 & 0.699244 & 0.518799 & 0.52748 & 0.765246 & 0.482143 & 0.606566 \\
6 & 0.781728 & 0.485283 & 0.524784 & 0.727676 & 0.49804 & 0.574781 \\
7 & 0.679856 & 0.481142 & 0.610627 & 0.697268 & 0.459933 & 0.542571 \\
8 & 0.712404 & 0.513218 & 0.505951 & 0.675901 & 0.468635 & 0.484024 \\
9 & 0.730591 & 0.58208 & 0.469869 & 0.660774 & 0.436629 & 0.506654 \\
10 & 0.677004 & 0.525617 & 0.502877 & 0.752461 & 0.45137 & 0.609843 \\
25 & 0.796089 & 0.497763 & 0.481045 & 0.78752 & 0.450377 & 0.588283 \\
50 & 0.984639 & 0.49728 & 0.558462 & 0.983334 & 0.545853 & 0.548067 \\
\hline
\end{tabular}

Tabela 5.1: e-,p- e q-valores para $H_{0}$ verdadeira $(\mathrm{N}=20,40$

\begin{tabular}{c|c|c|c|c|c|c} 
& \multicolumn{3}{|c|}{$N=60$} & \multicolumn{3}{c}{$N=100$} \\
\hline \hline$\theta$ & e-valor & p-valor & q-valor & e-valor & p-valor & q-valor \\
\hline \hline 1 & 0.749383 & 0.493024 & 0.608918 & 0.777473 & 0.539721 & 0.623876 \\
2 & 0.735574 & 0.55664 & 0.473259 & 0.677263 & 0.513985 & 0.505479 \\
3 & 0.76775 & 0.484063 & 0.56799 & 0.693188 & 0.420926 & 0.554276 \\
4 & 0.690172 & 0.481837 & 0.587969 & 0.741786 & 0.521095 & 0.591912 \\
5 & 0.691083 & 0.477329 & 0.502564 & 0.745216 & 0.510298 & 0.588356 \\
6 & 0.724908 & 0.450741 & 0.632785 & 0.753056 & 0.461581 & 0.601309 \\
7 & 0.71778 & 0.45677 & 0.538484 & 0.706427 & 0.540139 & 0.56292 \\
8 & 0.72203 & 0.484918 & 0.551893 & 0.631801 & 0.464382 & 0.463343 \\
9 & 0.721679 & 0.485703 & 0.596442 & 0.64266 & 0.513517 & 0.489476 \\
10 & 0.679996 & 0.492941 & 0.575348 & 0.721626 & 0.493287 & 0.571269 \\
25 & 0.807679 & 0.460518 & 0.613569 & 0.842746 & 0.519697 & 0.584002 \\
50 & 0.983778 & 0.476936 & 0.519676 & 1 & 0.446327 & 0.576726 \\
\hline
\end{tabular}

Tabela 5.2: e-,p- e q-valores para $H_{0}$ verdadeira $(\mathrm{N}=60,100)$

É digno de nota que todos os testes tenderam a se comportar melhor com o aumento do valor de $\theta$. Esse fato vai ao encontro da intuição; é razoável imaginar que, quando a taxa de ocorrência do processo aumenta, as perturbações devidas a $\lambda$ se tornam mais perceptíveis. Além disso, conforme indicado no Capítulo 1, a simetria da distribuição aumenta conforme $\theta$ cresce.

Com o crescimento de $N$, os testes frequentistas ganham qualidade, e o TRVG aumenta a precisão numa velocidade maior do que o teste de Consul-Famoye. Isso também é condizente com as definições dos testes, uma vez que as considerações assintóticas necessárias à construção do teste de Consul-Famoye dependem também do crescimento de $\theta$.

Os valores de evidência dados pelo FBST, por sua vez, não se mostram sensíveis ao tamanho particular da amostra utilizada, o que é reflexo da ausência de hipóteses assintóticas na definição do seu cálculo.

Dentre os três valores de evidência aqui considerados, os calculados via FBST apresentaram desempenho bastante superior aos testes frequentistas quando $H_{0}$ é verdadeira. As regiões do espaço com desempenho mais fraco, para os três testes, é a de $\theta \leq 10$.

Em seguida, serão analisados os resultados dos três testes para outros valores de $\lambda$. 


\subsubsection{Desempenho para $\lambda \neq 0$}

Para analisar o comportamento dos valores de evidência sobre outros valores de $\lambda$, foi fixado o valor de $\theta=50$, e nos gráficos da Figura 5.7 são apresentados os resultados.

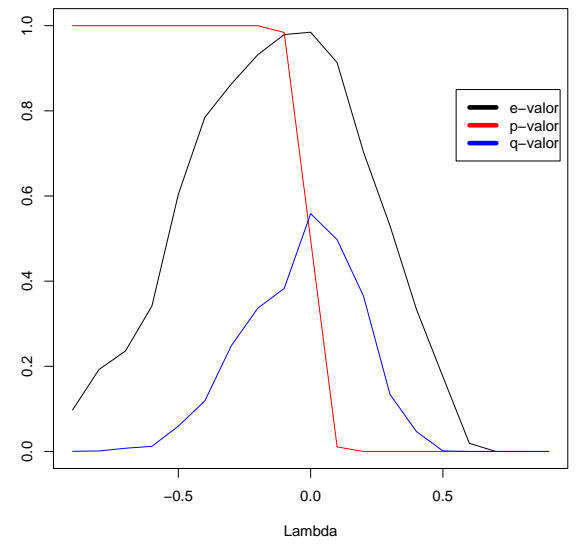

(a) $\mathrm{N}=20$

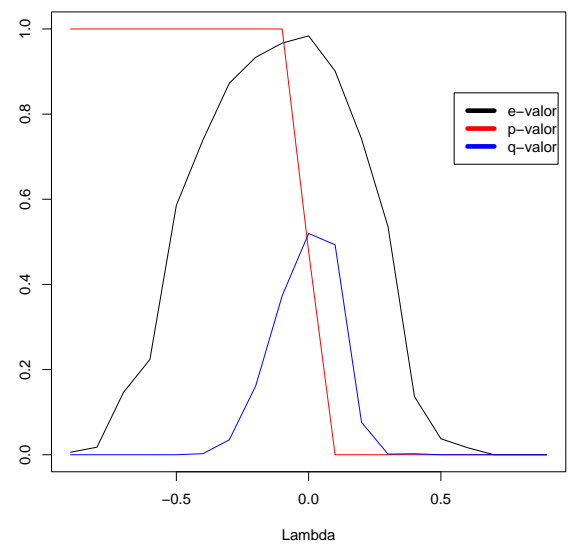

(c) $\mathrm{N}=60$

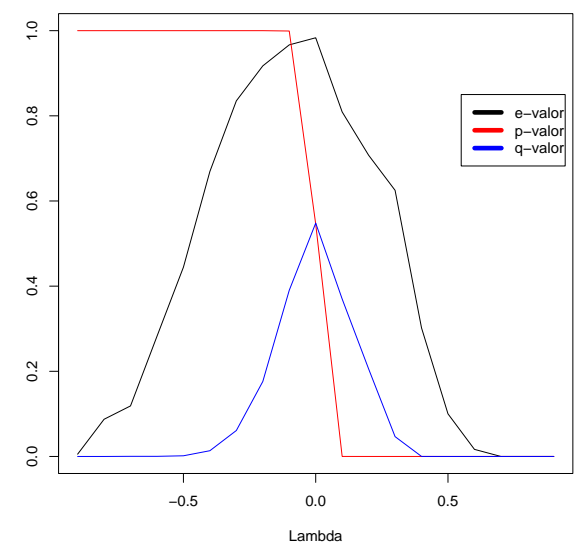

(b) $\mathrm{N}=40$



(d) $\mathrm{N}=100$

Figura 5.7: Valores de evidência em favor da hipótese para $\theta=50$ 
Os valores dados pelo teste de Consul-Famoye comportam-se quase que dicotomicamente: quando $\lambda$ cresce à direita de 0 , o p-valor decresce rapidamente para 0; o mesmo acontece quando $\lambda$ se distancia de 0 pelo lado esquerdo.

As curvas representativas do e-valor e do q-valor têm o comportamento esperado, com o máximo em $\lambda=\lambda_{0}$, e decrescendo conforme $\lambda$ se distancia desse valor. A curva calculada pelo FBST, porém, é diferenciável ao longo de todo o espaço, ao contrário das curvas calculadas pelo TRVG.

Nota-se também nessas duas curvas a presença de uma anomalia, quando $N=100$ e $\lambda=0.6$. Uma vez que os testes foram, para cada tamanho de amostra, recalculados diversas vezes, a hipótese de uma anomalia

estatística da amostra utilizada pode ser descartada. Trata-se assim de alguma característica da DPG, refletida na função de verossimilhança (que é utilizada por esses dois testes, mas não pelo teste de Consul-Famoye).

Foi observado um melhor desempenho do FBST quando $\lambda=\lambda_{0}$; quando comparados os resultados de cada teste com $\lambda$ livre, percebe-se que o e-valor é um majorante do q-valor. As naturezas das medidas são diferentes, o que se vê refletido nesse fato. No entanto, a curva definida pelo FBST possui propriedades desejáveis, tais como a diferenciabilidade. 


\section{Capítulo 6}

\section{Conclusão}

O objetivo principal do trabalho era definir o FBST com suas principais propriedades, e aplicá-lo ao teste do parâmetro extra na Distribuição de Poisson Generalizada. Para efeito de comparação foram utilizados os testes de Consul-Famoye e da Razão de Verossimilhanças Generalizada.

No capítulo 2 a importância do modelo da DPG foi demonstrada, pela sua aplicação em diversos problemas de probabilidade; em suma, essa distribuição surge como uma maneira de modelar processos estocásticos com probabilidades não homogêneas de ocorrência. Vimos alguns exemplos de processos desse tipo.

No capítulo 3, foram listadas algumas propriedades do FBST desejáveis em um procedimento de cálculo de evidência, e algumas dessas propriedades se refletiram nos resultados obtidos. Em primeiro lugar, de acordo com o princípio da verossimilhança, toda a informação amostral utilizada no teste veio da função de verossimilhança; por outro lado, nenhuma informação a priori foi utilizada para garantir igualdade de condições nos cálculos das medidas de evidência. Notou-se também a independência da medida do e-valor com relação ao tamanho da amostra, e a continuidade (e diferenciabilidade) da curva definida por essa medida.

A hipótese de interesse, $\lambda=\lambda_{0}=0$, representa a hipótese de que a distribuição de Poisson usual é adequada para os dados; em outras palavras, que os processos estocásticos modelados possuem taxas homogêneas no espaço e / ou tempo. O caráter preciso [23] da hipótese é plenamente aceito pela construção do FBST, mas para os testes frequentistas comparados a ele alguns problemas surgem. Para o teste de Consul-Famoye, a hipótese exata acaba sendo analisada como uma hipótese composta, do tipo $H_{0}: \lambda \leq \lambda_{0}$; o TRVG, por outro lado, se comporta fracamente quando o valor verdadeiro de $\lambda$ se aproxima do valor fixado por $H_{0}$.

No teste de Consul-Famoye, a hipótese alternativa composta garante a existência de um teste uniformemente mais poderoso [21], mas por outro lado o teste só tem poder alto na região definida por $H_{1}: \lambda>\lambda_{0}=0$. Por outro lado, o teste da Razão de Verossimilhanças Generalizada tem poder alto em toda a região complementar à definida por $H_{0}$, mas o custo por isso é um baixo valor de evidência mesmo quando $H_{0}$ é verdadeira, e a perda de algumas propriedades desejáveis para a curva de evidência.

O FBST, portanto, mostrou ter desempenho superior ao testar a hipótese precisa sobre o parâmetro extra da Poisson, e as propriedades do teste se confirmaram nos resultados dos cálculos. 
Apêndice A

Tabelas completas 







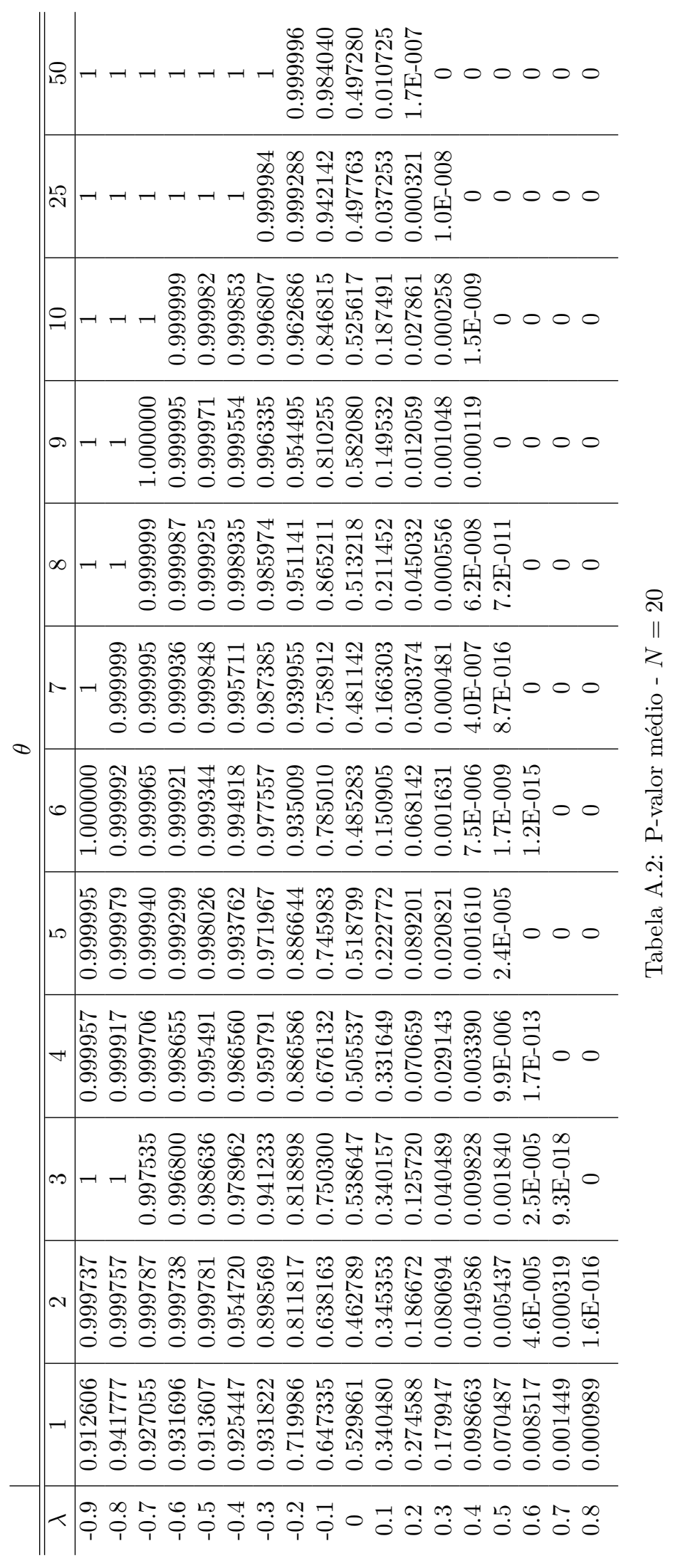




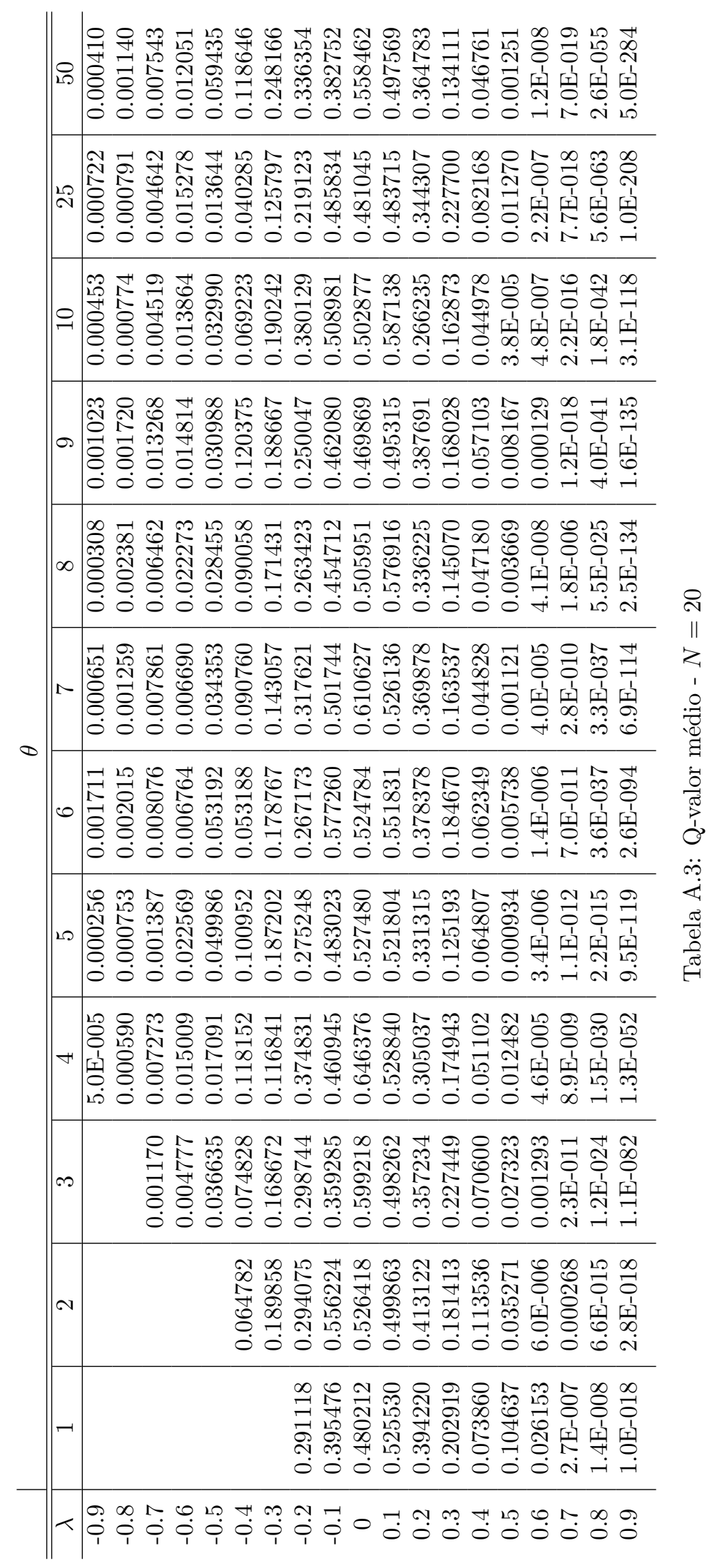




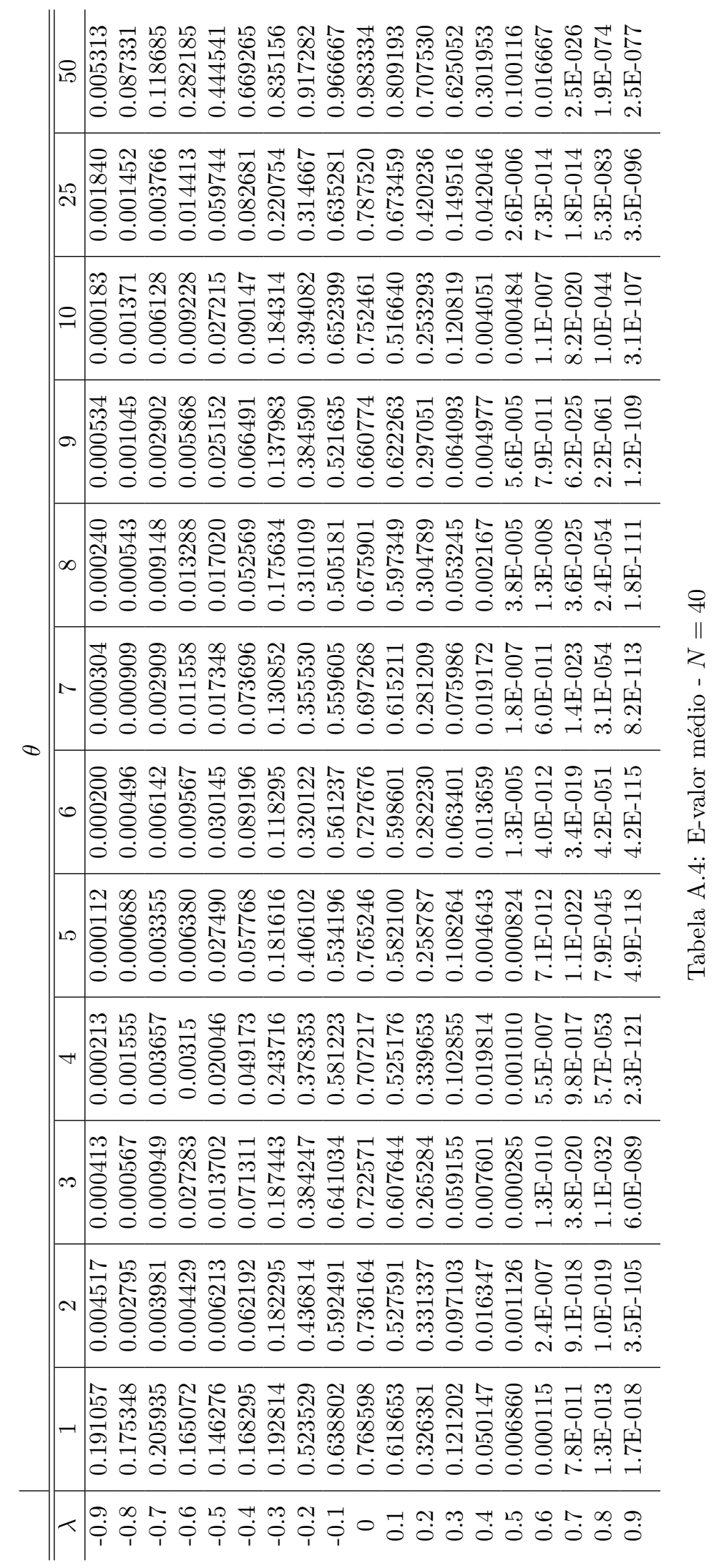




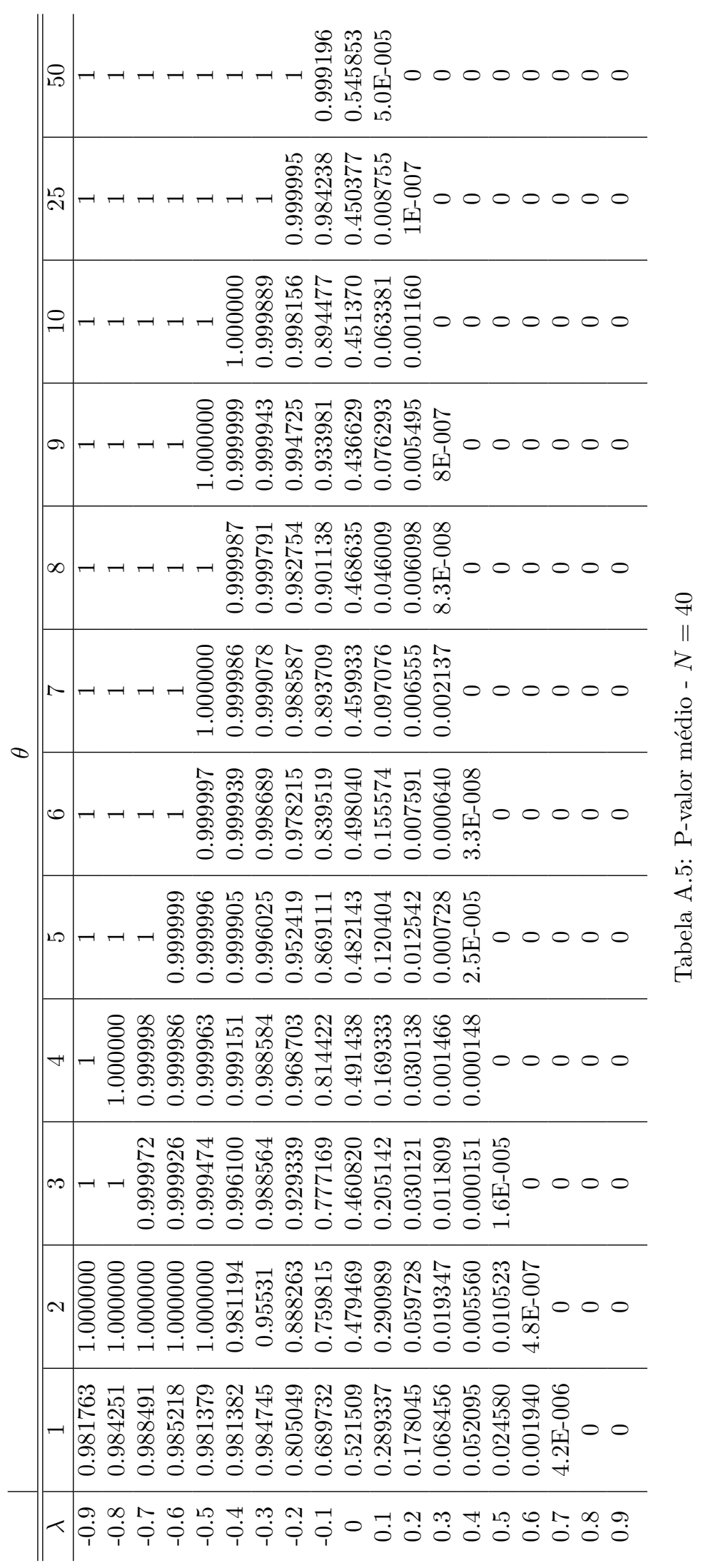




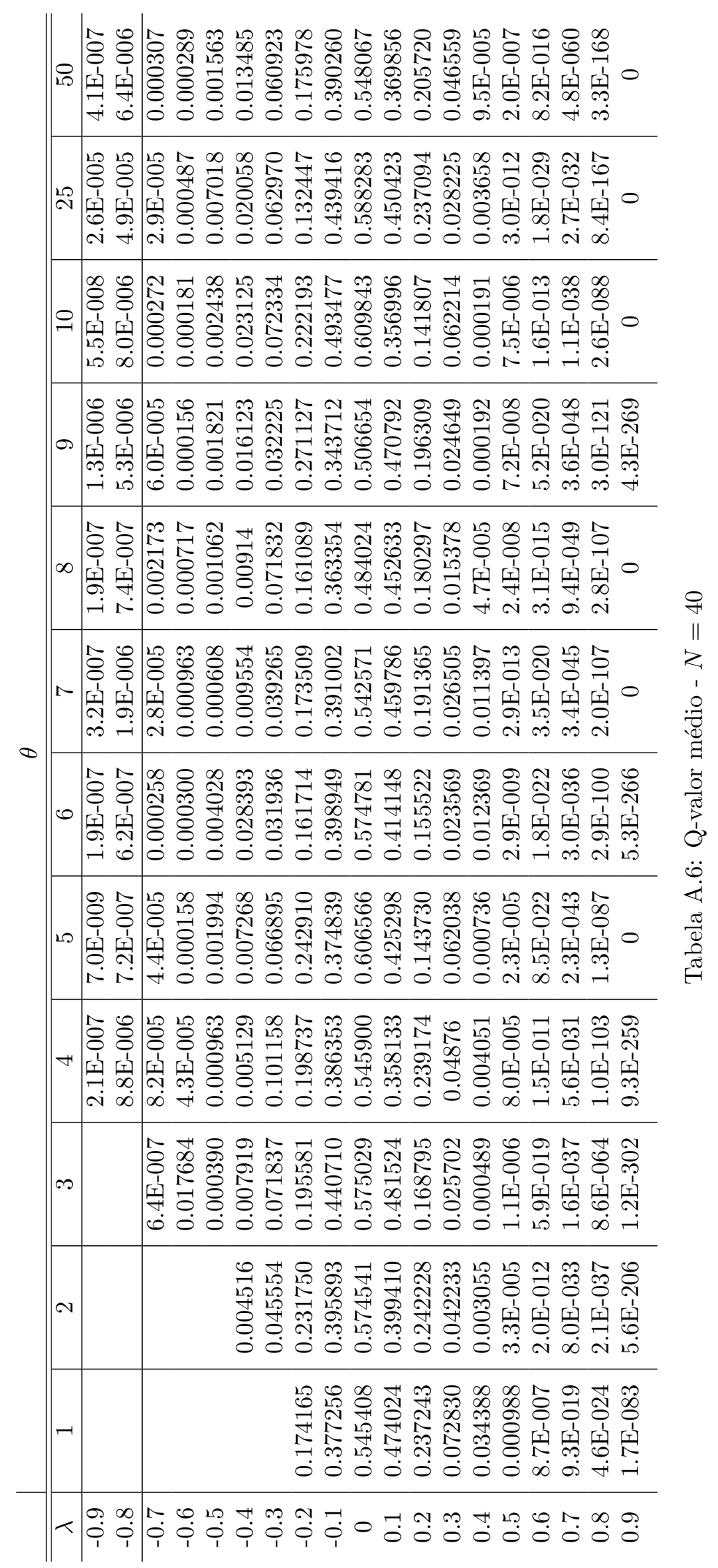




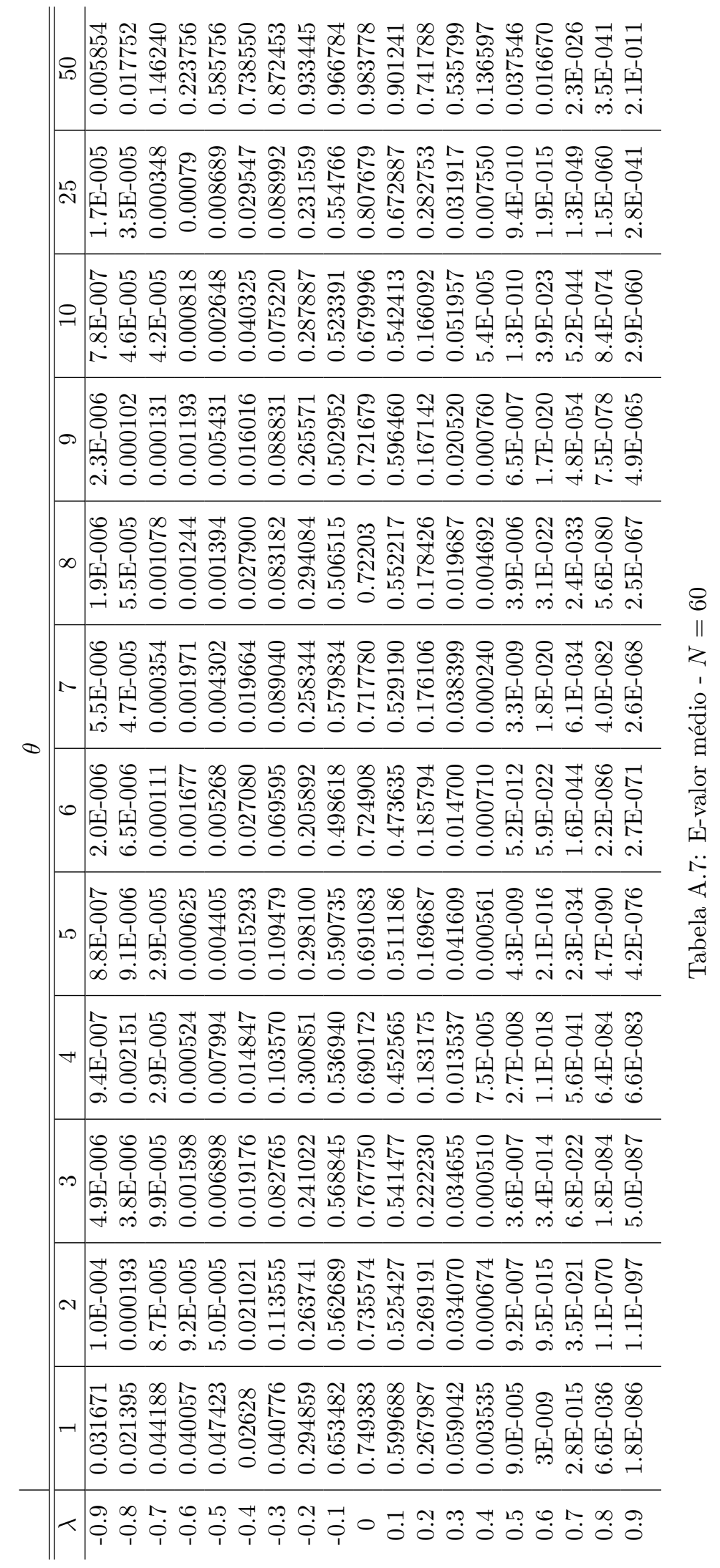




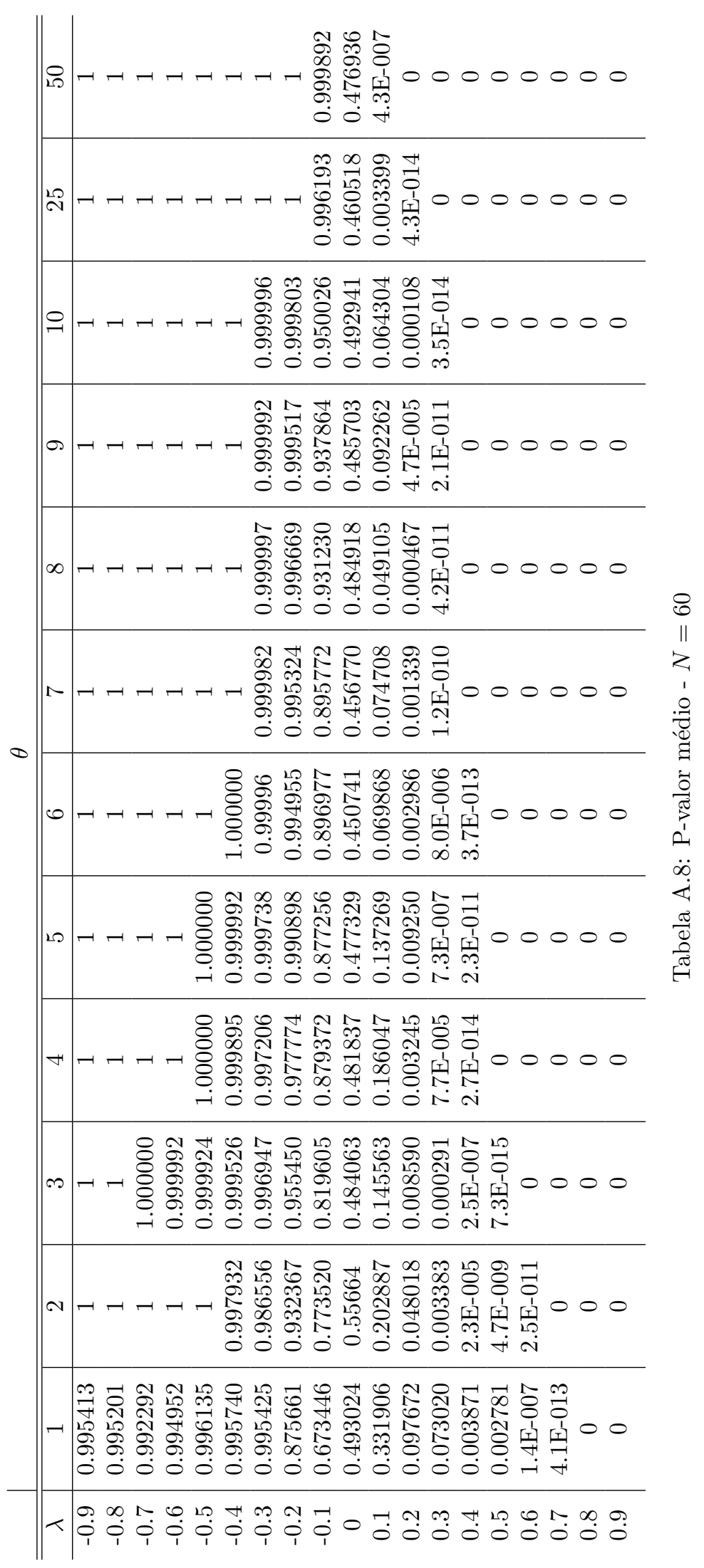




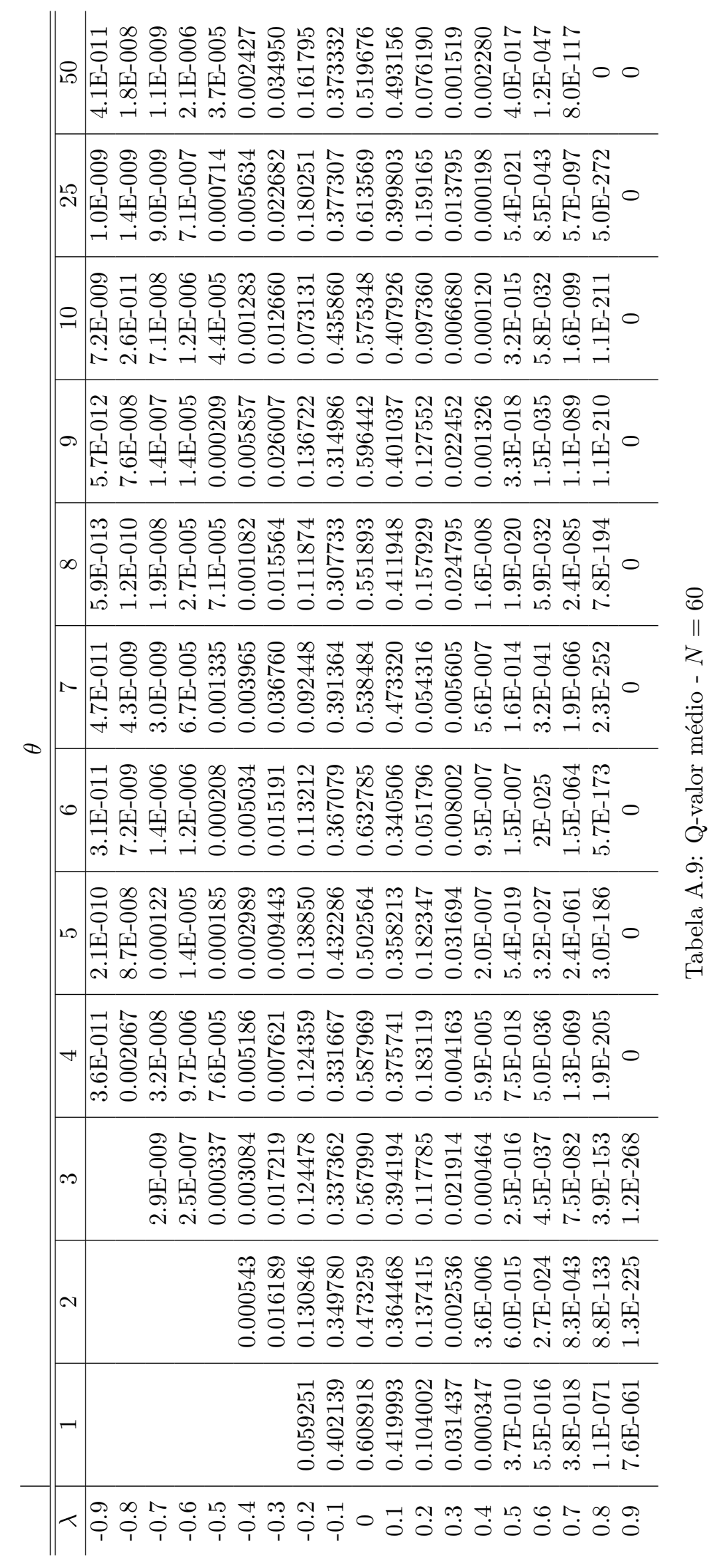




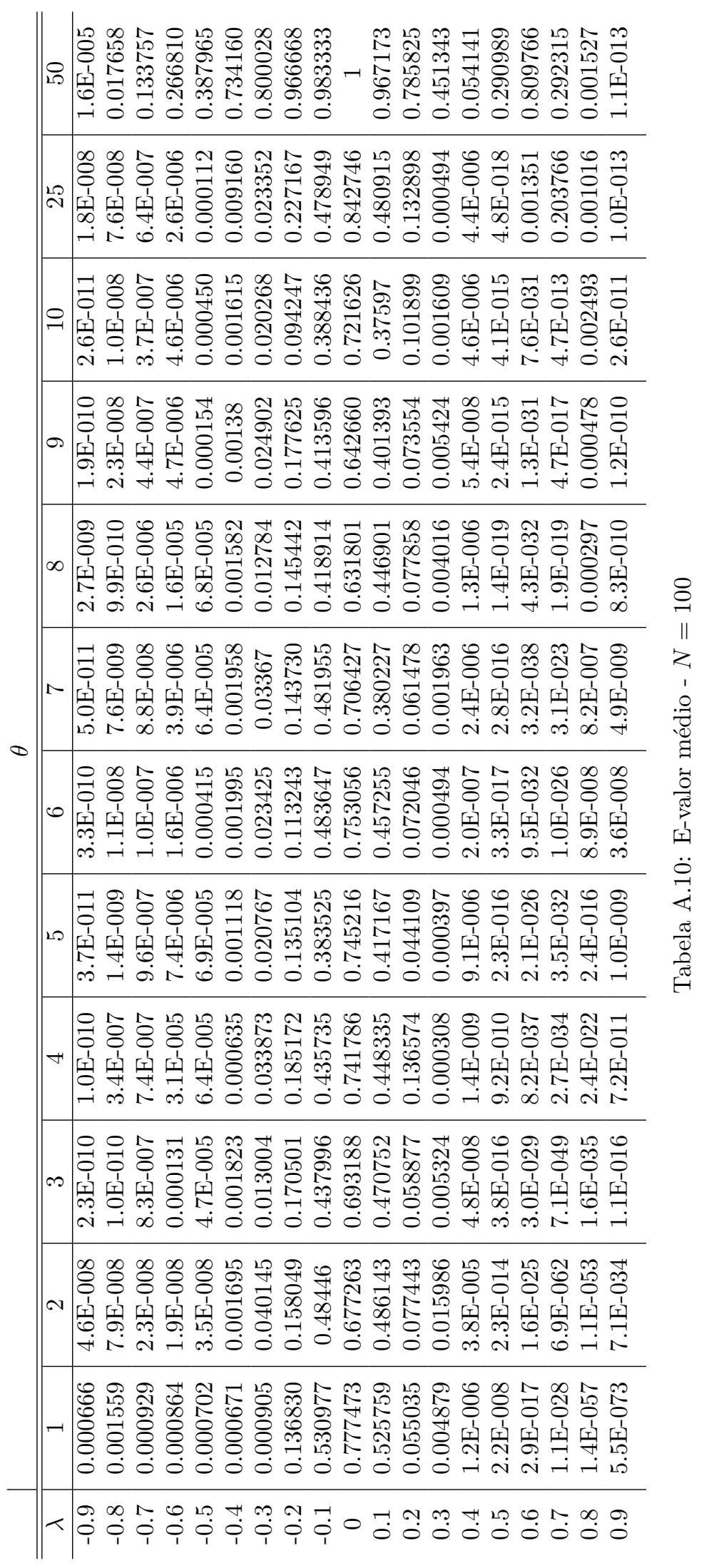









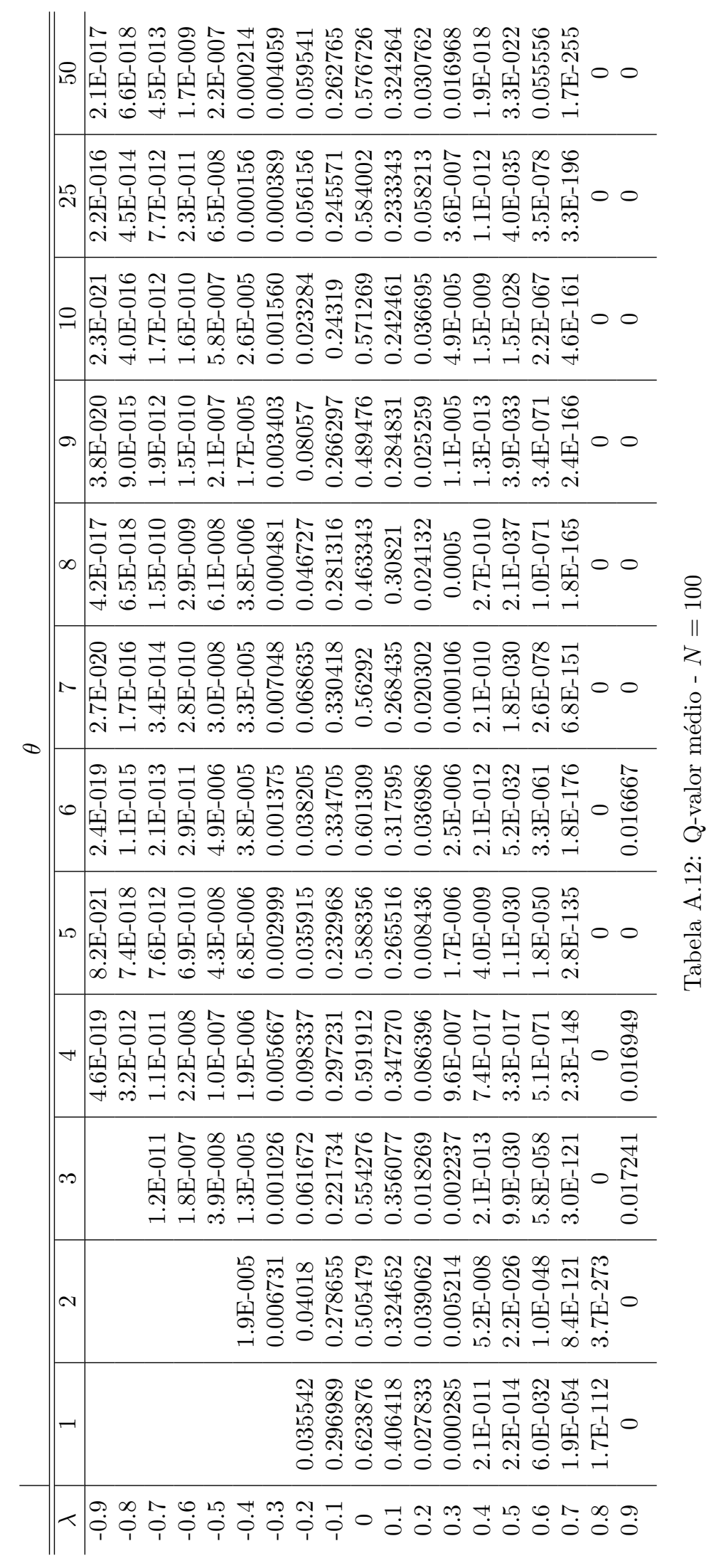




\section{Apêndice B}

\section{Código Fonte}

Nessa seção, apresentamos os códigos em $\mathrm{C}++$ utilizados ao longo do trabalho. O código foi escrito e compilado no Microsoft Visual Studio 6.0.

\section{B.1 clMersenneTwister - Gerador de números pseudo-aleatórios}

\section{B.1.1 clMersenneTwister.h}

A C-program for MT19937, with initialization improved 2002/1/26.

Coded by Takuji Nishimura and Makoto Matsumoto.

Before using, initialize the state by using init_genrand(seed)

or init_by_array(init_key, key_length).

Copyright (C) 1997 - 2002, Makoto Matsumoto and Takuji Nishimura, All rights reserved.

Redistribution and use in source and binary forms, with or without modification, are permitted provided that the following conditions are met:

1. Redistributions of source code must retain the above copyright notice, this list of conditions and the following disclaimer.

2. Redistributions in binary form must reproduce the above copyright notice, this list of conditions and the following disclaimer in the documentation and/or other materials provided with the distribution.

3. The names of its contributors may not be used to endorse or promote products derived from this software without specific prior written permission.

THIS SOFTWARE IS PROVIDED BY THE COPYRIGHT HOLDERS AND CONTRIBUTORS

"AS IS" AND ANY EXPRESS OR IMPLIED WARRANTIES, INCLUDING, BUT NOT

LIMITED TO, THE IMPLIED WARRANTIES OF MERCHANTABILITY AND FITNESS FOR 
A PARTICULAR PURPOSE ARE DISCLAIMED. IN NO EVENT SHALL THE COPYRIGHT OWNER OR CONTRIBUTORS BE LIABLE FOR ANY DIRECT, INDIRECT, INCIDENTAL, SPECIAL, EXEMPLARY, OR CONSEQUENTIAL DAMAGES (INCLUDING, BUT NOT LIMITED TO, PROCUREMENT OF SUBSTITUTE GOODS OR SERVICES; LOSS OF USE, DATA, OR PROFITS; OR BUSINESS INTERRUPTION) HOWEVER CAUSED AND ON ANY THEORY OF LIABILITY, WHETHER IN CONTRACT, STRICT LIABILITY, OR TORT (INCLUDING NEGLIGENCE OR OTHERWISE) ARISING IN ANY WAY OUT OF THE USE OF THIS SOFTWARE, EVEN IF ADVISED OF THE POSSIBILITY OF SUCH DAMAGE.

Any feedback is very welcome.

http://www.math.sci.hiroshima-u.ac.jp/ m-mat/MT/emt.html

$* /$ email: m-mat @ math.sci.hiroshima-u.ac.jp (remove space)

\#include <stdio.h>

// Period parameters

\#define N 624

\#define M 397

\#define MATRIX_A 0x9908b0dfUL // constant vector a

\#define UPPER_MASK 0x80000000UL // most significant w-r bits

\#define LOWER_MASK Ox7fffffffUL // least significant $r$ bits

class clMersenneTwister

\{

public:

clMersenneTwister ();

virtual clMersenneTwister();

void init_genrand(unsigned long); // initializes mt [N] with a seed

unsigned long genrand_int32(void); // generates a random number on [0,0xffffffff]-interval

double genrand_real2(void); // generates a random number on $[0,1$ )-real-interval

private:

unsigned long *mt; // the array for the state vector

int mti; // mti==N+1 means $\mathrm{mt}[\mathrm{N}]$ is not initialized

\} ;

\section{B.1.2 clMersenneTwister.cpp}

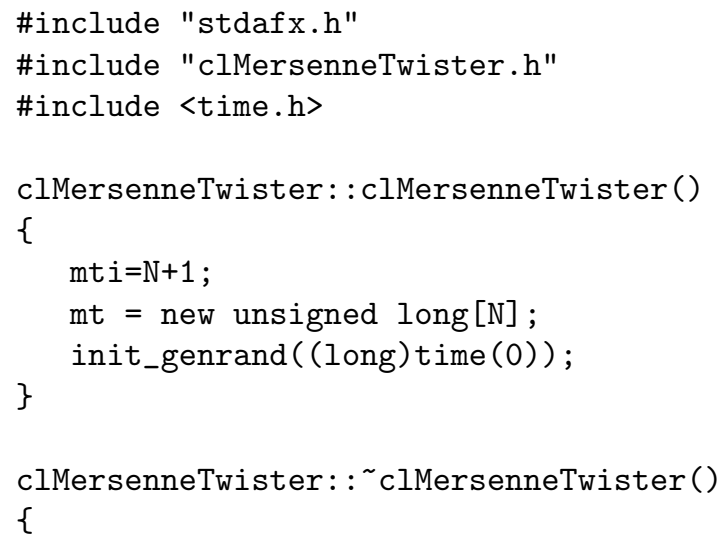


delete mt;

void clMersenneTwister: :init_genrand(unsigned long s)

\{

$\operatorname{mt}[0]=\mathrm{s} \&$ OxffffffffUL;

for $(\mathrm{mti}=1 ; \mathrm{mti}<\mathrm{N} ; \mathrm{mti++})\{$

$\mathrm{mt}[\mathrm{mti}]=$

(1812433253UL * (mt [mti-1] - (mt [mti-1] >> 30)) + mti);

/* See Knuth TAOCP Vol2. 3rd Ed. P.106 for multiplier. */

/* In the previous versions, MSBs of the seed affect */

/* only MSBs of the array mt[].

/*2002/01/09 modified by Makoto Matsumoto

mt [mti] \& = OxffffffffUL;

\}

/* for $>32$ bit machines */

\}

unsigned long clMersenneTwister: :genrand_int32(void)

\{

unsigned long y;

static unsigned long mag01[2] $=\{0 x 0 U L$, MATRIX_A $\}$;

$/ * \operatorname{mag} 01[\mathrm{x}]=\mathrm{x} *$ MATRIX_A for $\mathrm{x}=0,1 * /$

if (mti $>=N)\{/ *$ generate $N$ words at one time $* /$

int $\mathrm{kk}$;

if (mti $==\mathrm{N}+1$ ) /* if init_genrand() has not been called, */ init_genrand(5489UL); /* a default initial seed is used */

for $(\mathrm{kk}=0 ; \mathrm{kk}<\mathrm{N}-\mathrm{M} ; \mathrm{kk}++) \quad\{$

$\mathrm{y}=$ (mt $\left.[\mathrm{kk}] \& U P P E R \_M A S K\right) \mid\left(m t[k \mathrm{k}+1] \& L O W E R \_M A S K\right)$;

\}

$\mathrm{mt}[\mathrm{kk}]=\mathrm{mt}[\mathrm{kk}+\mathrm{M}] \wedge(\mathrm{y}>1) \wedge \operatorname{mag} 01[\mathrm{y} \&$ 0x1UL $]$;

for $(; \mathrm{kk}<\mathrm{N}-1 ; \mathrm{kk}++) \quad\{$

$\mathrm{y}=$ (mt [kk] \&UPPER_MASK) $\mid(\mathrm{mt}[\mathrm{kk}+1]$ \&LOWER_MASK $)$;

\}

$\mathrm{mt}[\mathrm{kk}]=\operatorname{mt}[\mathrm{kk}+(\mathrm{M}-\mathrm{N})] \wedge(\mathrm{y}>>1) \wedge \operatorname{mag} 01[\mathrm{y} \& 0 \mathrm{x} 1 \mathrm{UL}]$;

$\mathrm{y}=$ (mt $\left.[\mathrm{N}-1] \& U P P E R \_M A S K\right) \mid\left(m t[0] \& L O W E R \_M A S K\right) ;$

$\mathrm{mt}[\mathrm{N}-1]=\mathrm{mt}[\mathrm{M}-1] \wedge(\mathrm{y}>1) \wedge \operatorname{mag} 01[\mathrm{y} \& \mathrm{0x} 1 \mathrm{UL}]$;

$\mathrm{mti}=0$;

\}

$\mathrm{y}=\mathrm{mt}[\mathrm{mti}++]$

/* Tempering */

$\mathrm{y} \wedge$ = (y $\gg 11)$;

$\mathrm{y}^{\wedge}=(\mathrm{y} \ll 7)$ \& 0x9d2c5680UL;

$\mathrm{y}^{\wedge}=(\mathrm{y} \ll<15)$ \& 0xefc60000UL;

$\mathrm{y}^{\wedge}=(\mathrm{y}>>18)$;

return y;

\} 


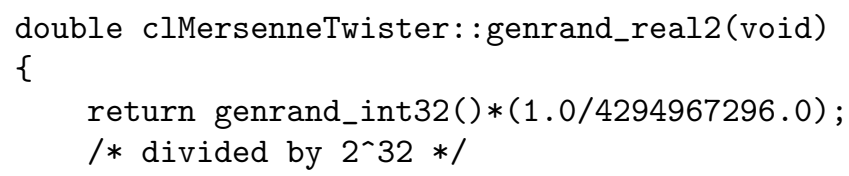

\section{B.2 clDPG - Implementa a Distribuição de Poisson Generalizada}

\section{B.2.1 clDPG.h}

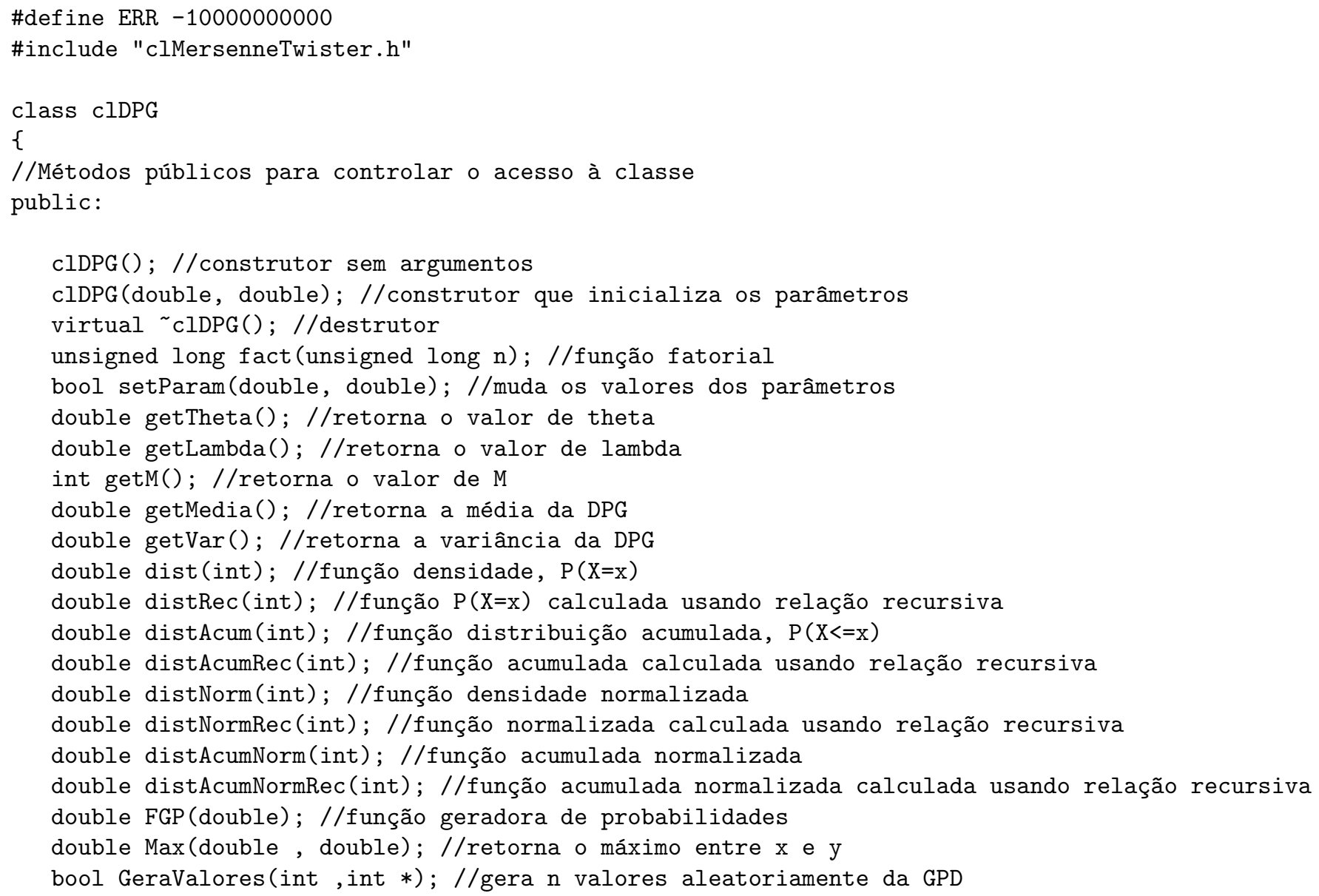




\section{B.2.2 clDPG.cpp}






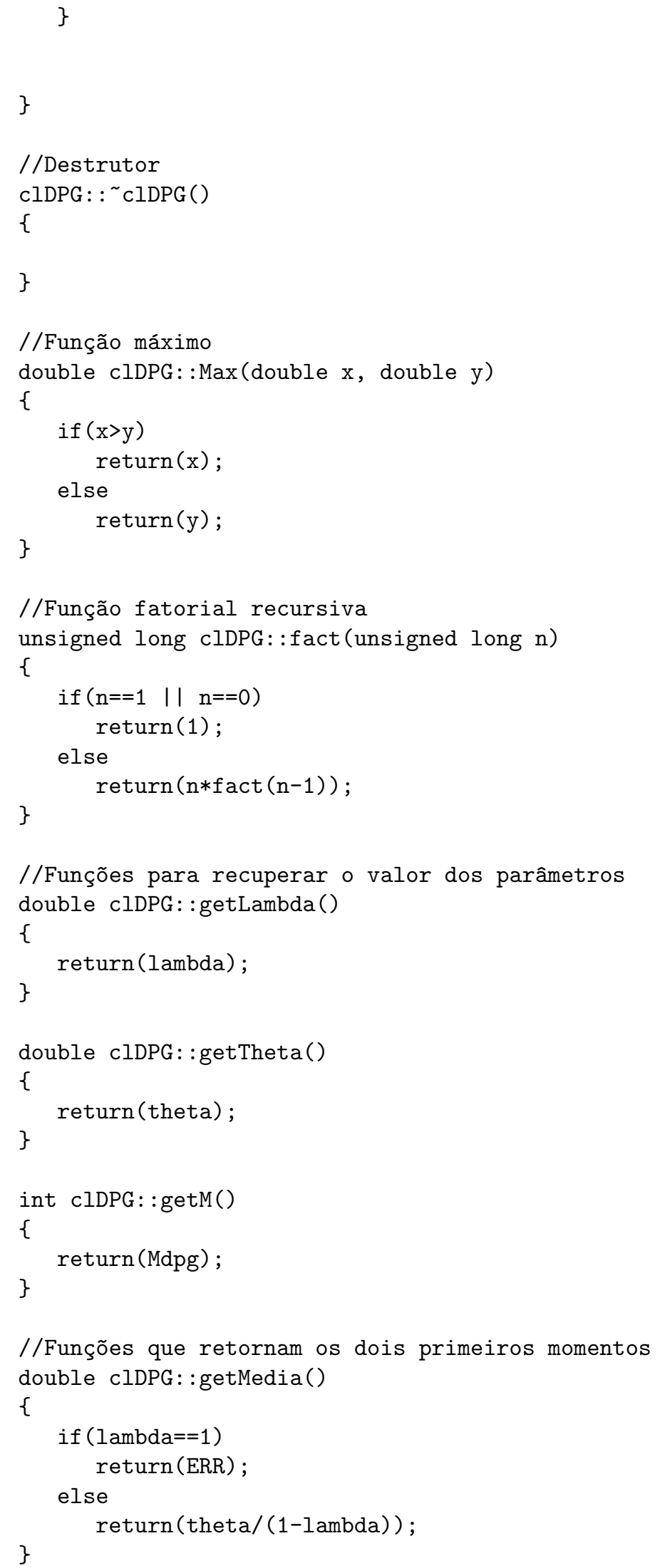









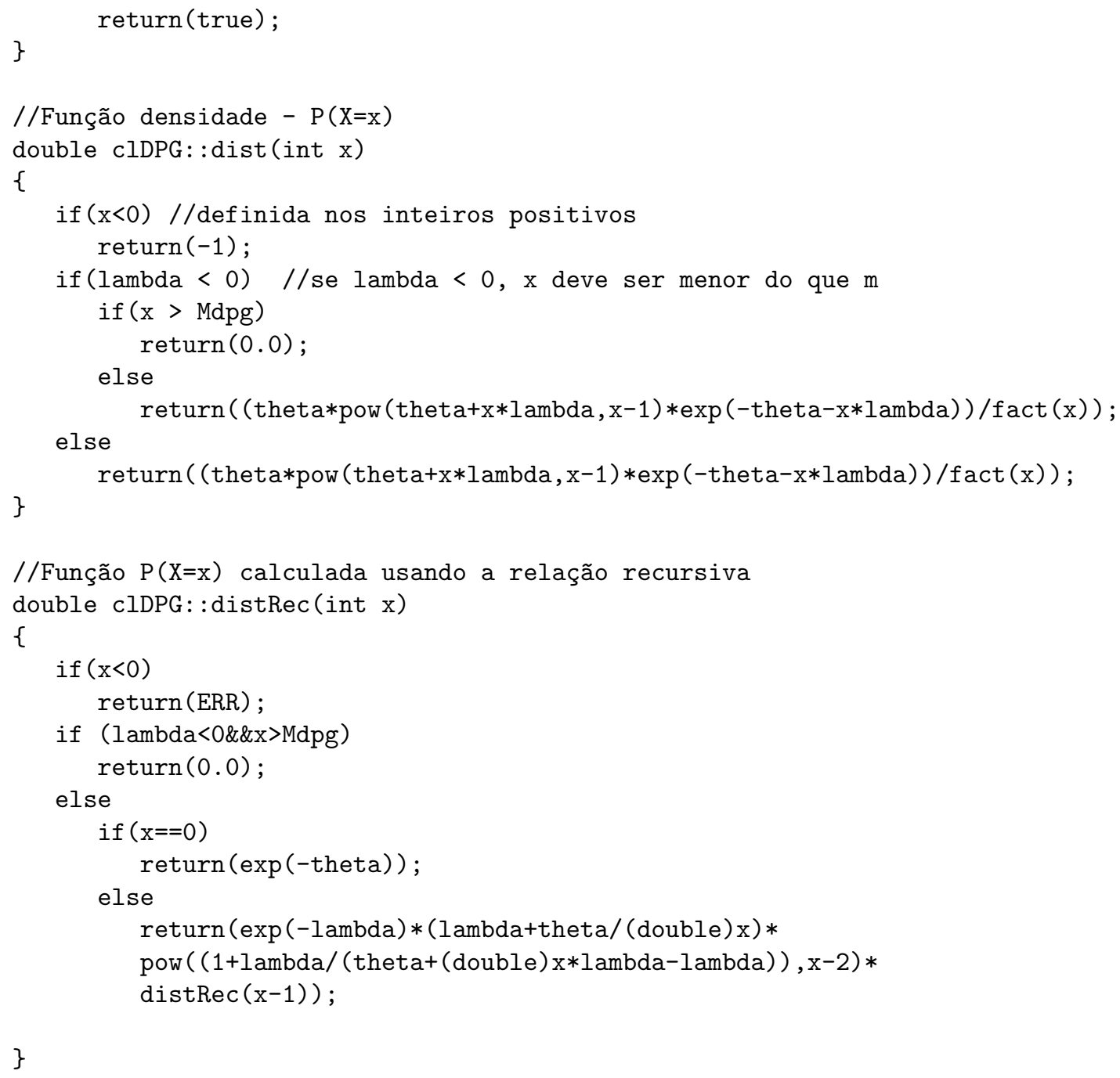




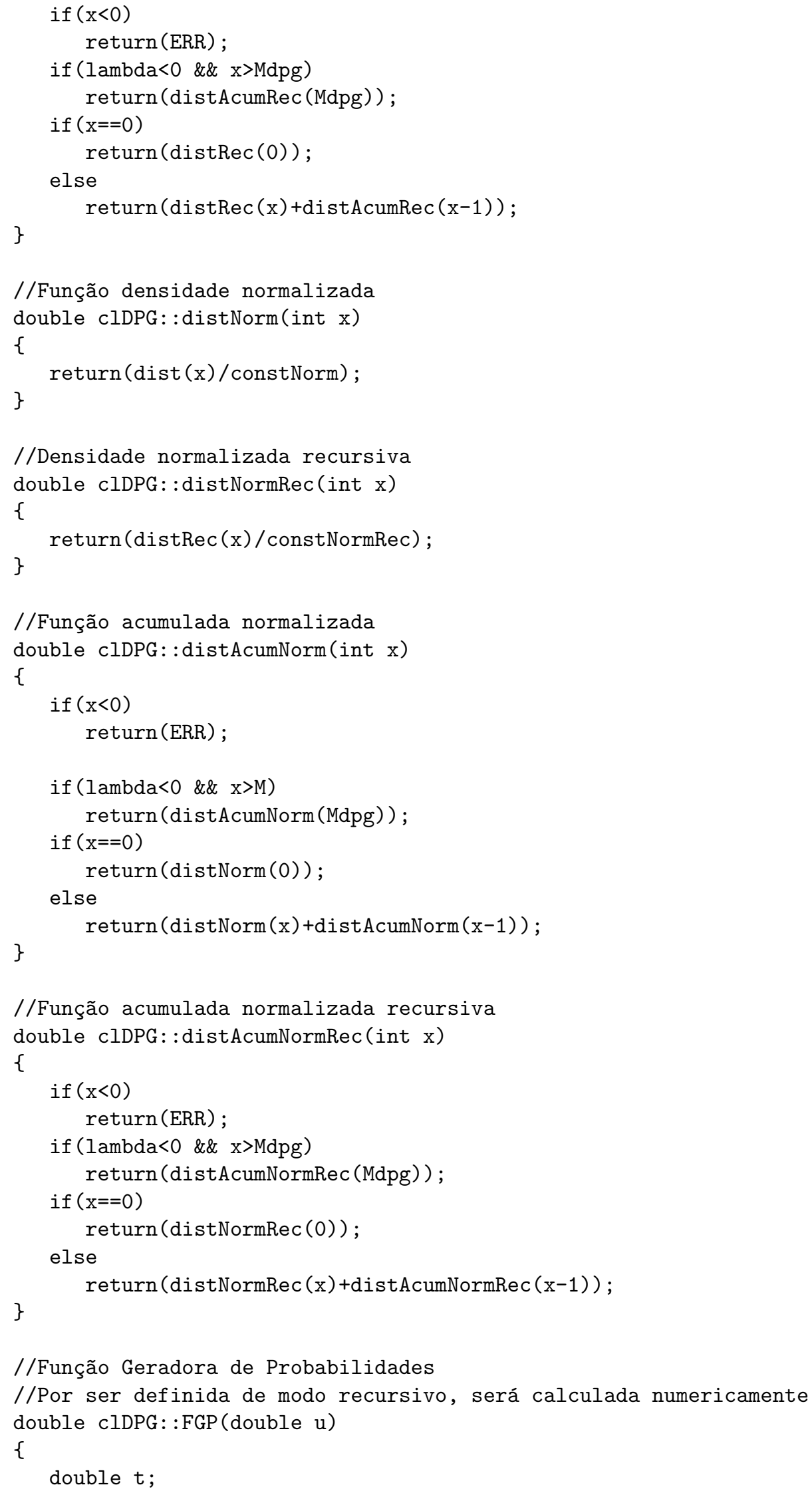


//Método de Newton-Raphson:

//Procura o ponto inicial t tal que $t-u * \exp (\operatorname{lambda}(t-1))$ e -t-u*exp(lambda(-t-1)) tenham //sinais opostos, para garantir que a raiz da função se encontra em [-t,t].

//A cada atualização de $t$, testa para ver se a função exponencial está retornando //um número válido

//_FPCLASS_SNAN, _FPCLASS_QNAN e _fpclass são utilizadas para controle de erros $\mathrm{t}=10$;

if $($ fpplass $(\exp (\operatorname{lambda} *(t-1)))==$ _FPCLASS_SNAN ||

_fpclass $(\exp (\operatorname{lambda} *(t-1)))==\_$FPCLASS_QNAN ||

_fpclass $(\exp (\operatorname{lambda} *(t-1)))==$ FPCLASS_NINF ||

_fpclass $(\exp (\operatorname{lambda} *(t-1)))==$ _FPCLASS_PINF)

return (ERR);

while $((\mathrm{t}-\mathrm{u} * \exp (\operatorname{lambda} *(\mathrm{t}-1))) *(-\mathrm{t}-\mathrm{u} * \exp (\operatorname{lambda} *(-\mathrm{t}-1)))>=0)$

\{

$\mathrm{t}=\mathrm{t} * 2$;

if (_fpclass $(\exp (\operatorname{lambda} *(t-1)))==$ FPCLASS_SNAN ||

_fpclass $(\exp (\operatorname{lambda} *(t-1)))==$ FPCLASS_QNAN I|

_fpclass $(\exp (\operatorname{lambda} *(t-1)))==$ _FPCLASS_NINF ||

_fpclass $(\exp (\operatorname{lambda} *(t-1)))==$ _FPCLASS_PINF)

\} return (ERR);

//Utilizando o método de Newton-Raphson unidimensional para encontrar o $t$ que satisfaça $/ / \mathrm{a}$ equação $\mathrm{t}=\mathrm{u} * \exp (\operatorname{lambda} *(t-1))$

while $(f a b s(t-u * \exp (\operatorname{lambda} *(t-1)))>1 e-15)$

\{

$\mathrm{t}=\mathrm{t}-(\mathrm{t}-\mathrm{u} * \exp (\operatorname{lambda} *(\mathrm{t}-1))) /(1-\mathrm{lambda} * \mathrm{u} * \exp (\mathrm{lambda} *(\mathrm{t}-1)))$;

if $\left(\_f p c l a s s(\exp (\operatorname{lambda} *(t-1)))==\right.$ FPCLASS_SNAN ||

_fpclass $(\exp (\operatorname{lambda} *(t-1)))==$ _FPCLASS_QNAN ||

_fpclass $(\exp (\operatorname{lambda} *(t-1)))==$ FPCLASS_NINF ||

_fpclass $(\exp (\operatorname{lambda} *(t-1)))==$ _FPCLASS_PINF)

\}

return (ERR);

//testa se o cálculo de $\exp ($ theta*(t-1)) retorna um valor numérico

if (_fpclass $(\exp ($ theta $*(t-1)))==$ FPCLASS_SNAN ||

_fpclass $(\exp (\operatorname{theta} *(t-1)))==$ _FPCLASS_QNAN ||_fpclass $(\exp (\operatorname{theta} *(t-1)))==$ _FPCLASS_NINF

||_fpclass $(\exp (\operatorname{theta} *(t-1)))==$ FPCLASS_PINF $)$

return (ERR);

$\operatorname{return}(\exp (\operatorname{theta*}(t-1)))$;

\} ;

//Função para simular valores da DPG

bool clDPG: :GeraValores(int $\mathrm{n}$, int *piRetValues)

\{

//o algoritmo é descrito no texto da dissertação

double S,P,U, c;

double omega $=\exp (-$ lambda $)$;

int $i, X$;

for $(i=0 ; i<n ; i++)$

\{

$\mathrm{X}=0$;

$\mathrm{S}=\exp ($-theta) ; 


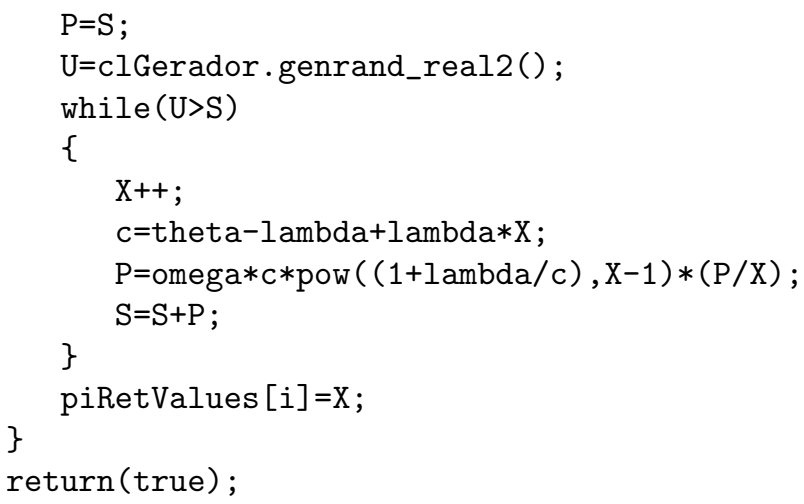

\section{B.3 clCF - Teste de Consul-Famoye}

\section{B.3.1 clCF.h}

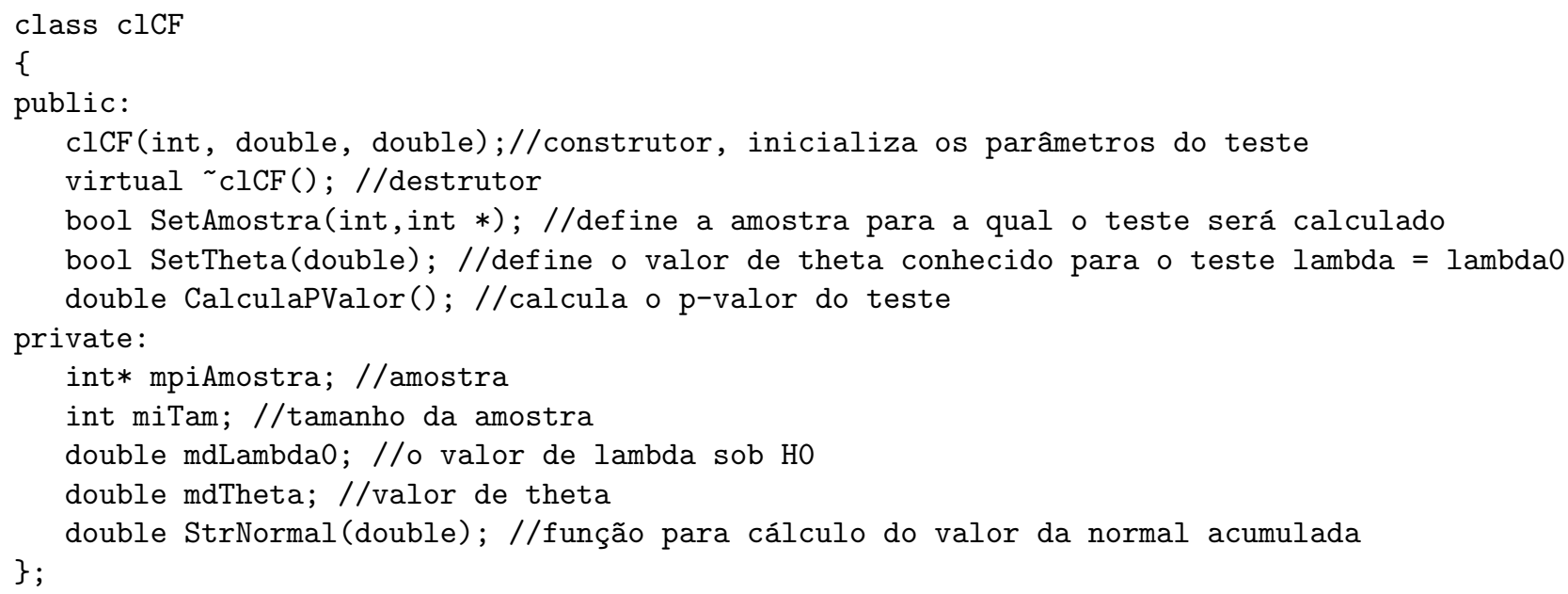

\section{B.3.2 clCF.cpp}

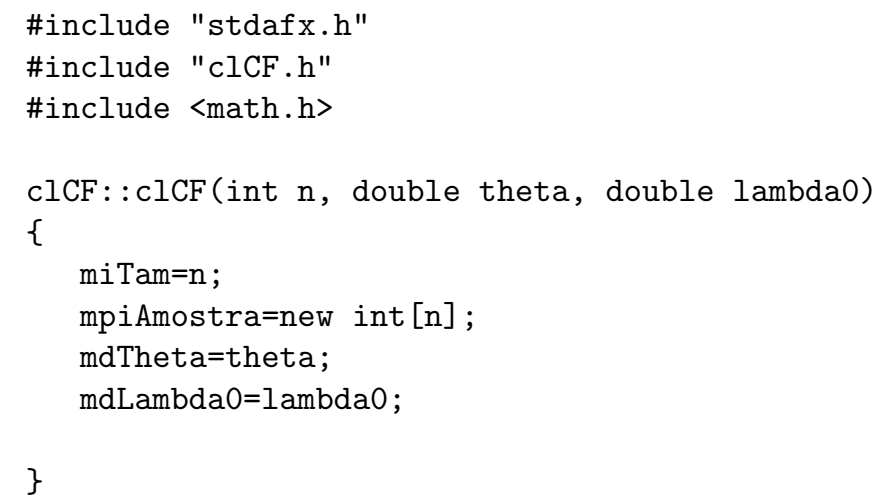




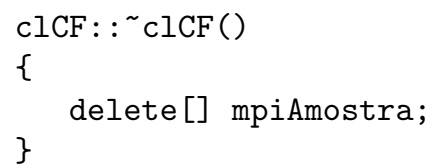


$\mathrm{C}=($ dXbarra-mdTheta $*(1 /(1-\mathrm{mdLambda} 0))) / \operatorname{sqrt}($ mdTheta $*$ pow $(1-\mathrm{mdLambda} 0,-3.0) / \mathrm{miTam})$; return(StrNormal (C));

\section{B.4 clRVG - Teste de razão de verossimilhanças generalizado}

\section{B.4.1 clRVG.h}

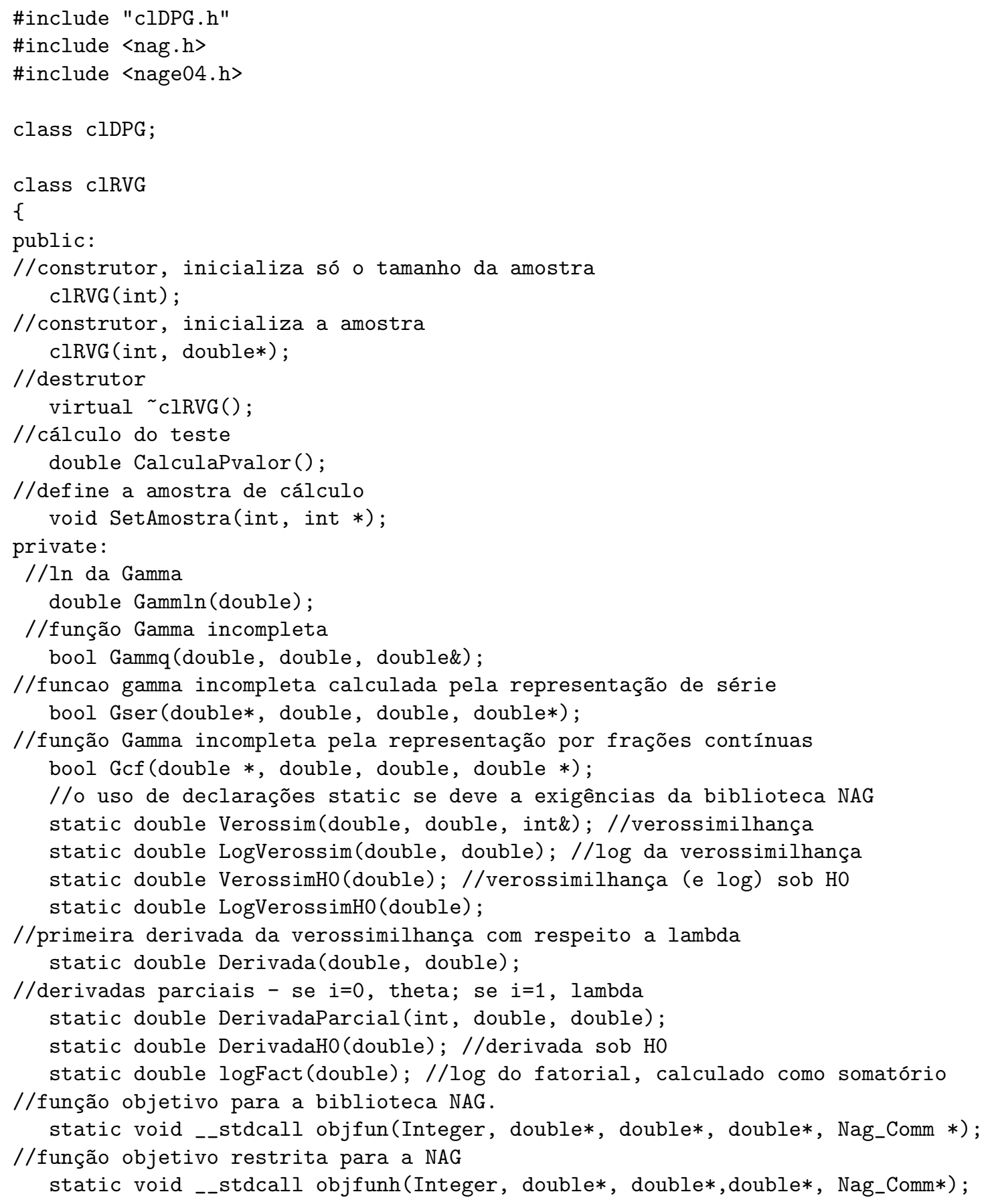


static clDPG clDistribuicao; //distribuição correspondent static int iTam;

static int* piAmostra; //amostra

\} ;

\section{B.4.2 clRVG.cpp}

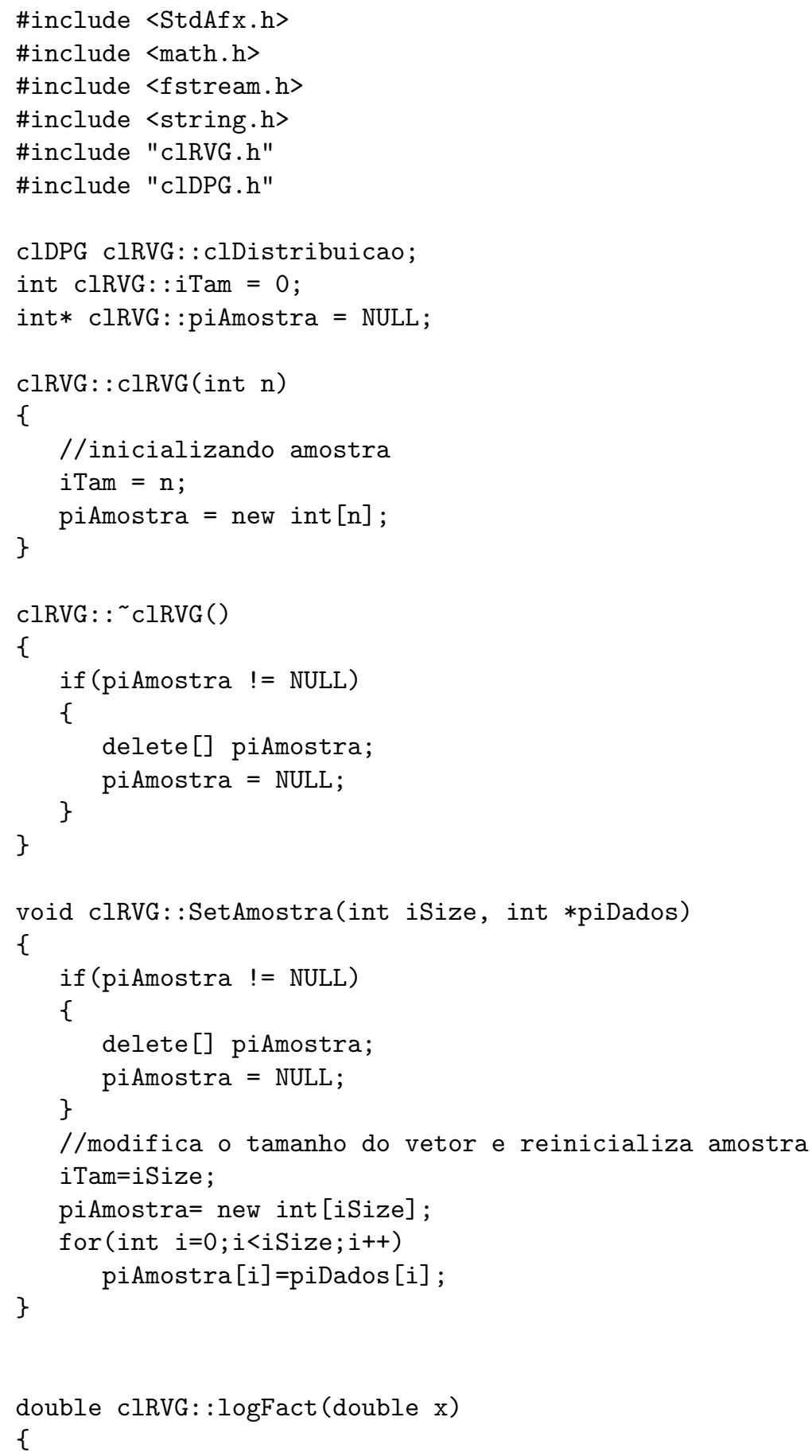









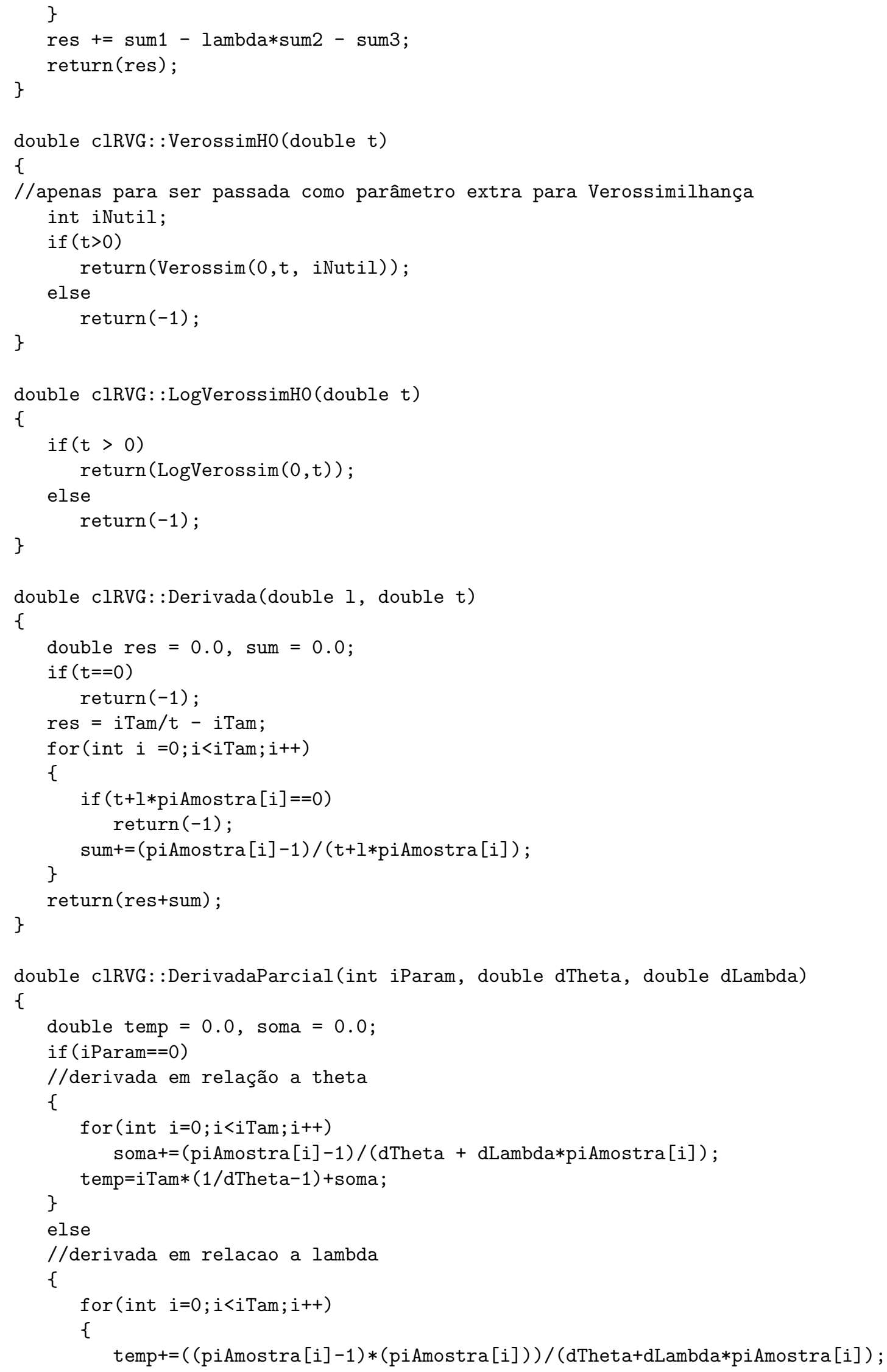




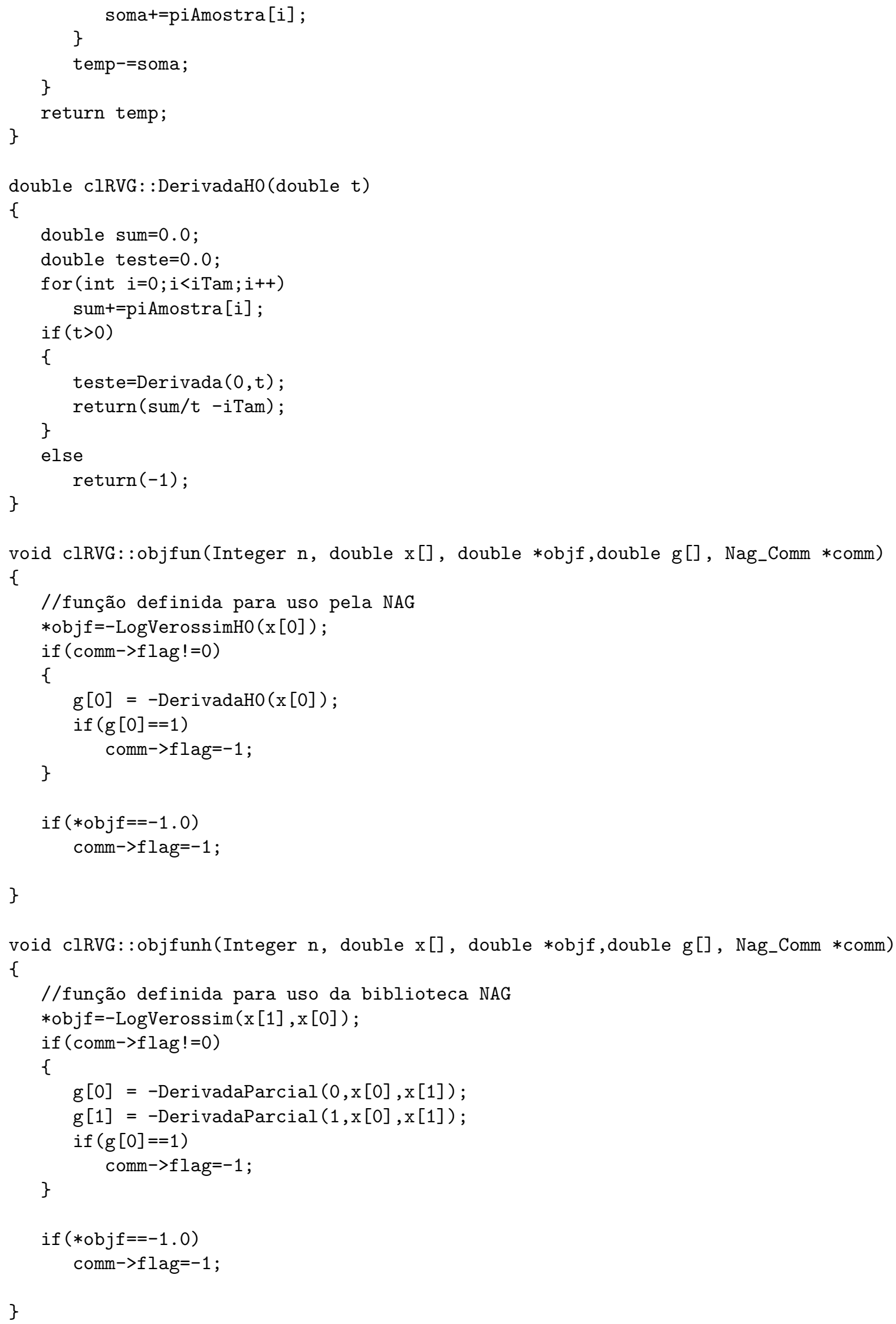









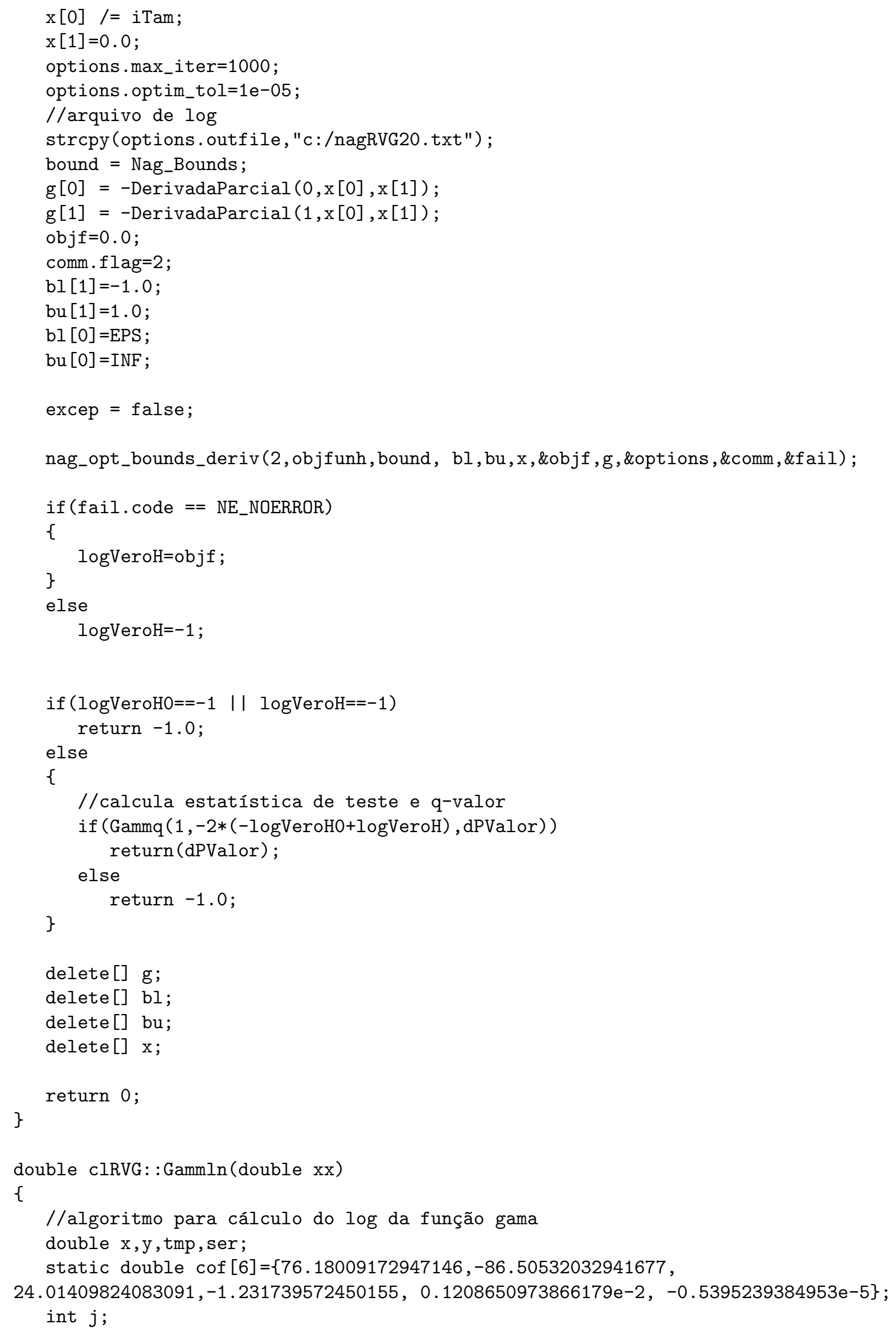




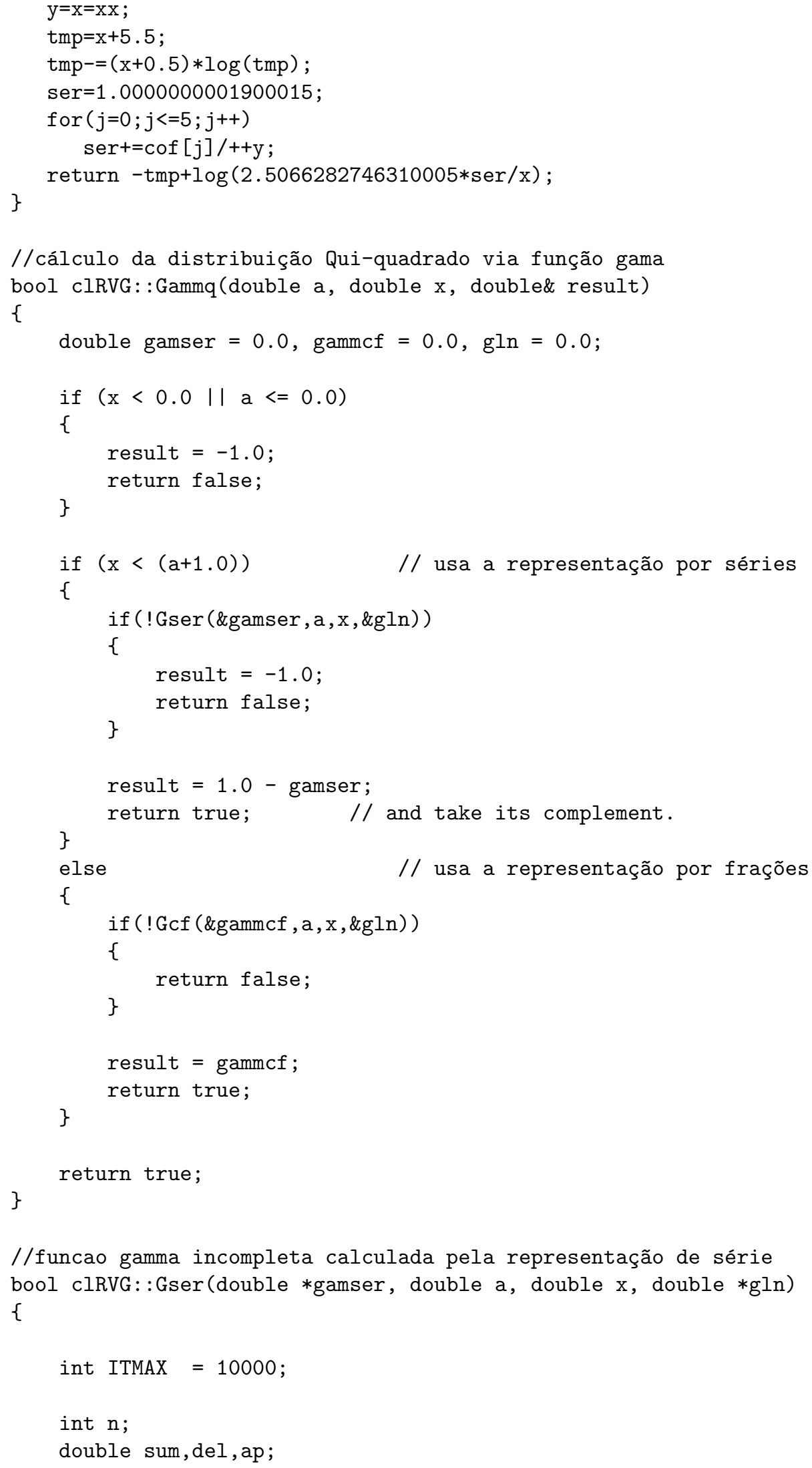




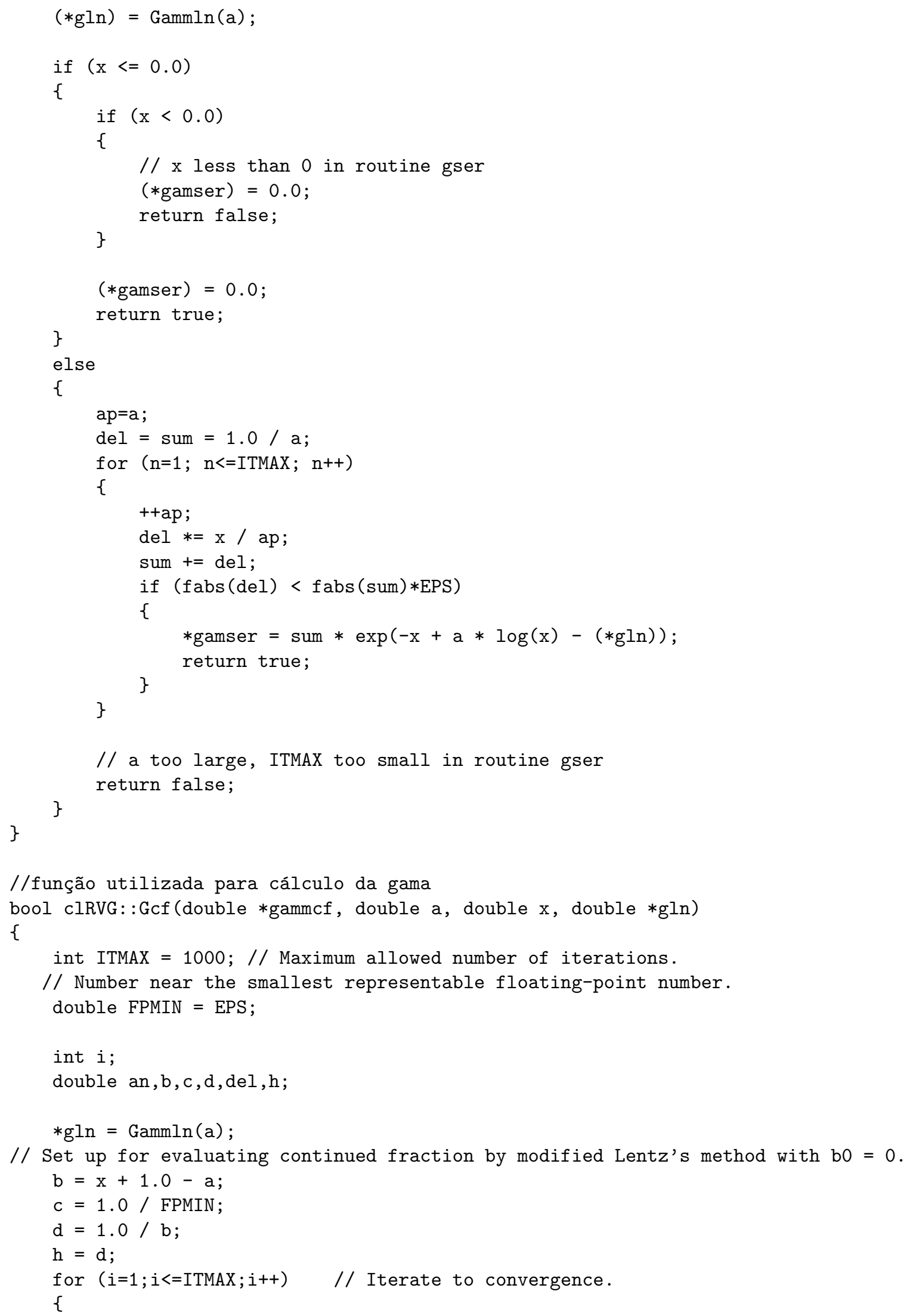




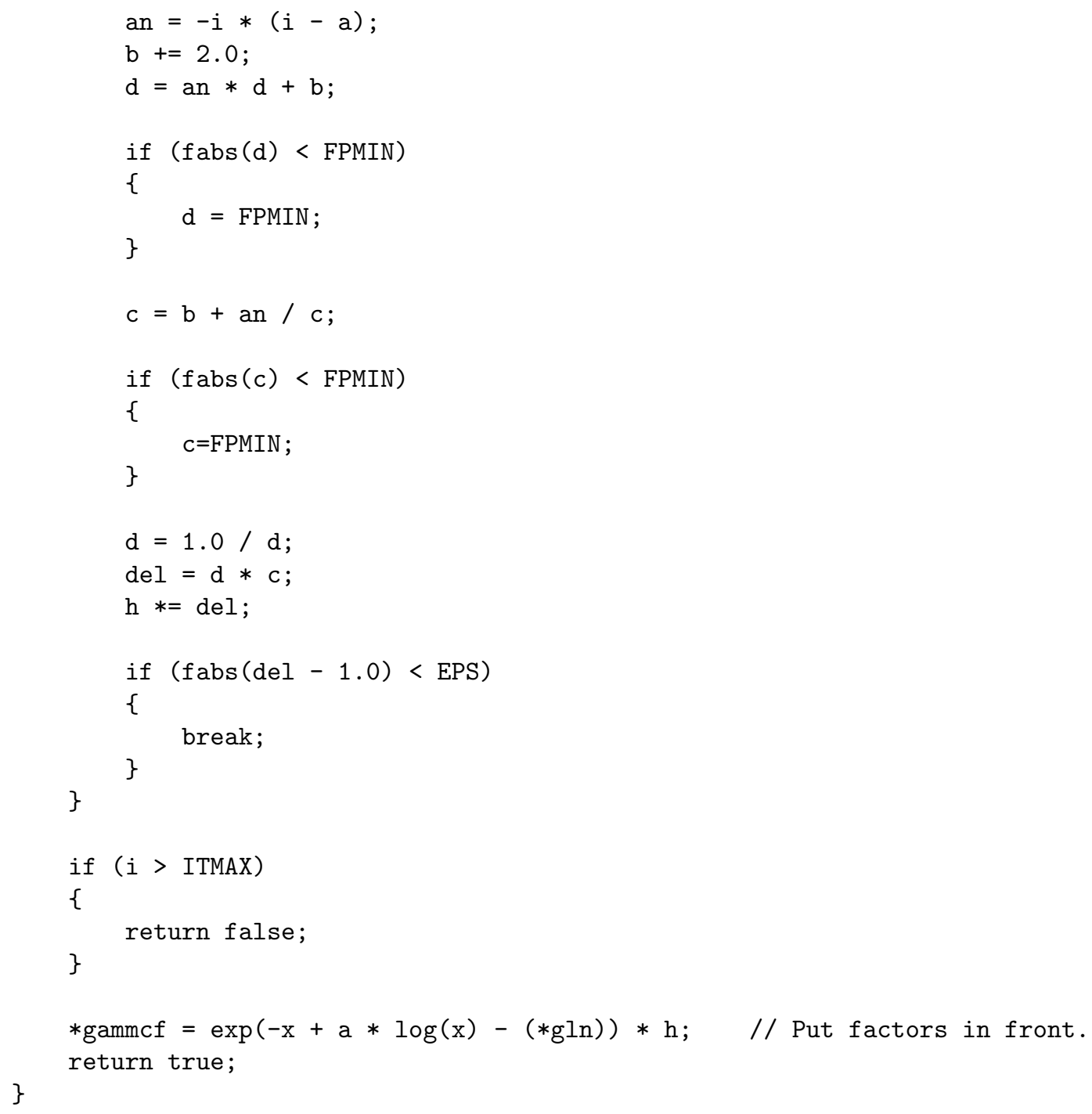

\section{B.5 clFBST - Full Bayesian Significance Test}

\section{B.5.1 clFBST.h}

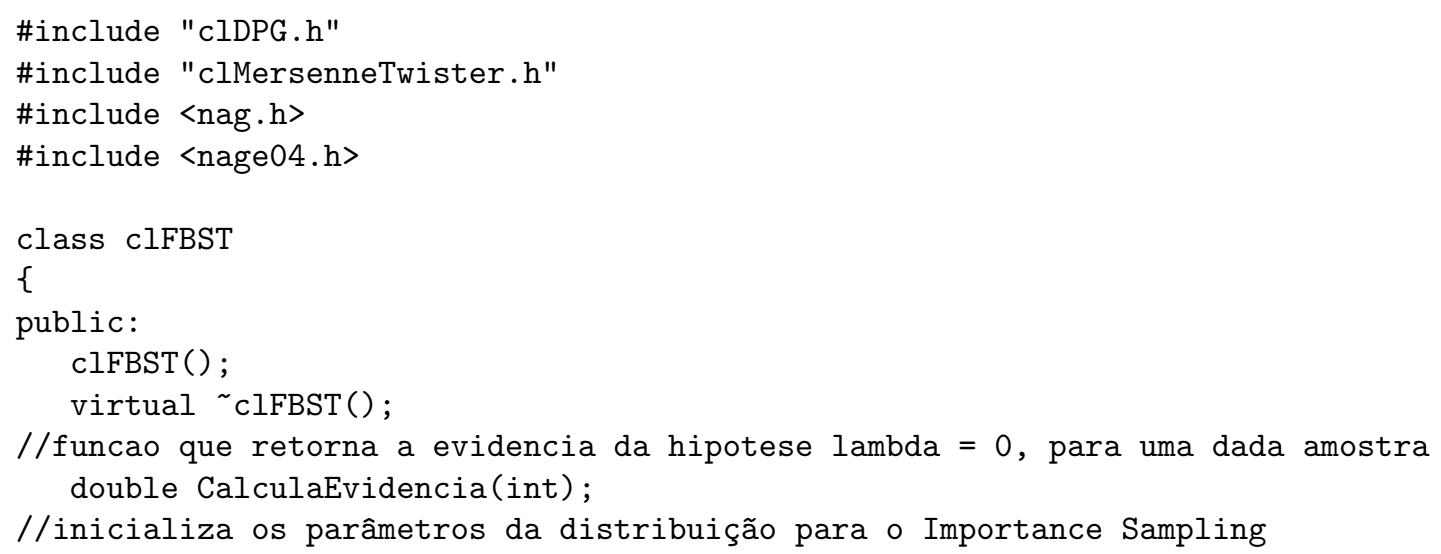


bool SetParamImportance(int, int, double);

//define a amostr

void SetAmostra(int, int*); a

private:

//DPG para ser usada no teste

static clDPG *clDistribuicao;

//Gerador de números aleatórios

clMersenneTwister clGerador;

double *pdSestrela; //s*

//valor da função surpresa em s* double dPosteriori;

//tamanho da amostra

static int iTam;

static int *piAmostra; //amostra

//parâmetros da Beta para Importance Sampling int $\mathrm{a}, \mathrm{b}$;

//parâmetro da Poisson para Importance Sampling double mi;

//calcula o valor de s* void Calculas();

//testa se o ponto (theta, lambda) pertence a $\mathrm{T}(\mathrm{s} *$ ) bool TestaRegiaoT(double, double);

$/ /$ posteriori (= função surpresa) static double Posteriori(double, double, int\&);

//Logaritmo neperiano da posteriori static double LogPosteriori(double, double);

$/ /$ posteriori sob $\mathrm{HO}:$ lambda $=0$ static double PosterioriHo(double);

//Logaritmo da posteriori sob HO static double LogPosterioriHo(double);

//derivada parcial dp/dTheta da log-posteriori static double Derivada(double, double);

//derivada d/dTheta da log-posteriori sob HO static double DerivadaHo(double);

//função objetivo para a biblioteca NAG. static void _-stdcall objfun(Integer, double*, double*, double*, Nag_Comm *);

//função para cálculo da integral double CalculaIntegral(int);

//função que gera pontos da distribuição do Importance Sampling bool ImportanceSampling (double *);

//densidade da distribuição de Importance Sampling double DensidadeImportanceSampling (double, double);

//gera valores da Beta bool GeraBeta(int, double *);

//gera valores da Exponencial bool GeraExponencial (int, double *);

//logaritmo da função gama static double LogGamma(double);

//função beta double Beta(double, double);

//fatorial static double fact(double);

//log do fatorial static double logFact(double); 


\section{B.5.2 clFBST.cpp}






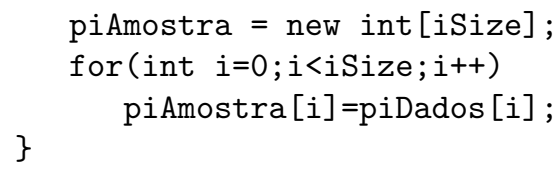




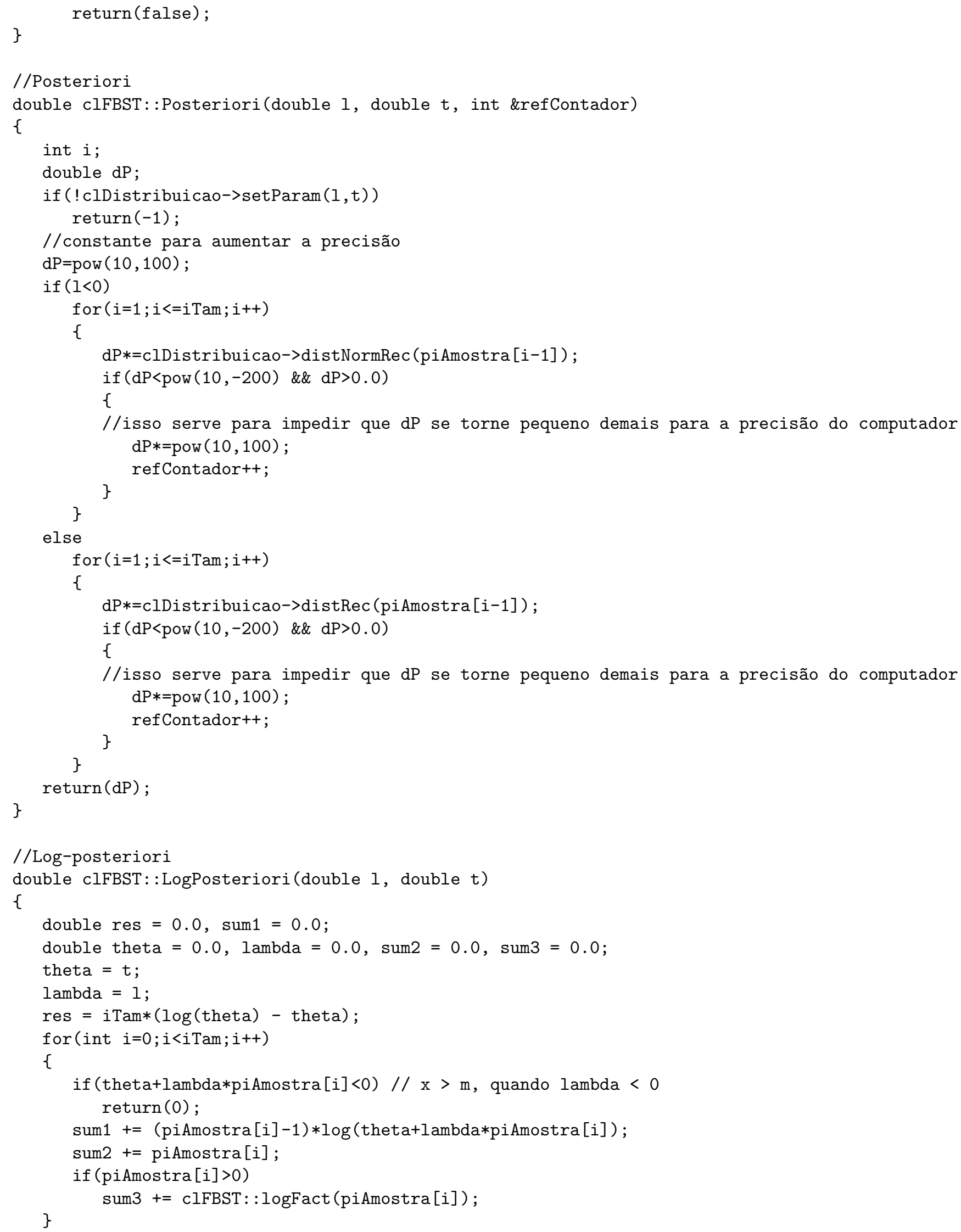




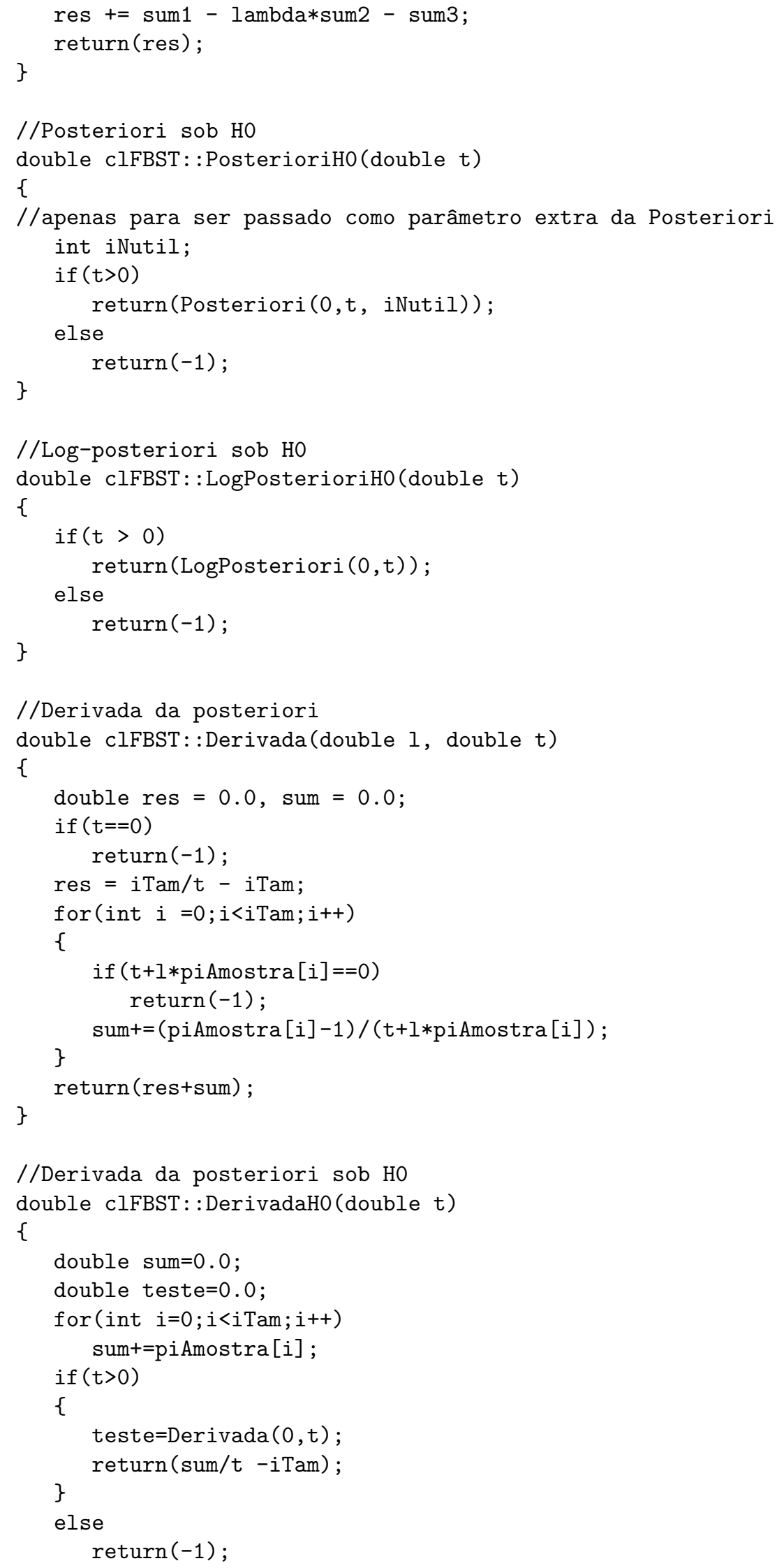


//Função objetivo para a NAG

void clFBST: :objfun(Integer $\mathrm{n}$, double $\mathrm{x}[]$, double *objf,double g[], Nag_Comm *comm) \{

*objf=-LogPosterioriHo (x[0]) ;

if $(\mathrm{comm}->$ flag $!=0)$

\{

$\mathrm{g}[0]=-\operatorname{DerivadaHO}(\mathrm{x}[0])$;

if $(\mathrm{g}[0]==1)$

\}

comm $->$ flag $=-1$;

if $(* o b j f==-1.0)$

comm $->$ flag $=-1$;

\}

//Calcula o s*

void clFBST: :Calculas()

\{

double objf, g, bl, bu, x;

Nag_Comm comm;

Nag_BoundType bound;

Nag_E04_Opt options;

static NagError fail;

fail print=FALSE;

//inicializando estrutura de opções

nag_opt_init(\&options);

options.deriv_check=FALSE;

options.local_search=FALSE;

options. linesearch_tol=0.5;

//arquivo de log da NAG

strcpy (options.outfile, "c:/nagFBST20.txt") ;

$\mathrm{bl}=\mathrm{EPS}$;

$\mathrm{bu}=\mathrm{INF}$;

$\mathrm{x}=0.0$;

//calculando chute inicial = média simples

for (int $i=0 ; i<i$ Tam; $i++$ )

$\mathrm{x}+=$ piAmostra $[\mathrm{i}]$;

$\mathrm{x} /=\mathrm{iTam}$;

bound = Nag_Bounds;

$\mathrm{g}=-\operatorname{DerivadaHO}(\mathrm{x})$;

objf $=0.0$;

comm.flag=2;

bool excep = false; 


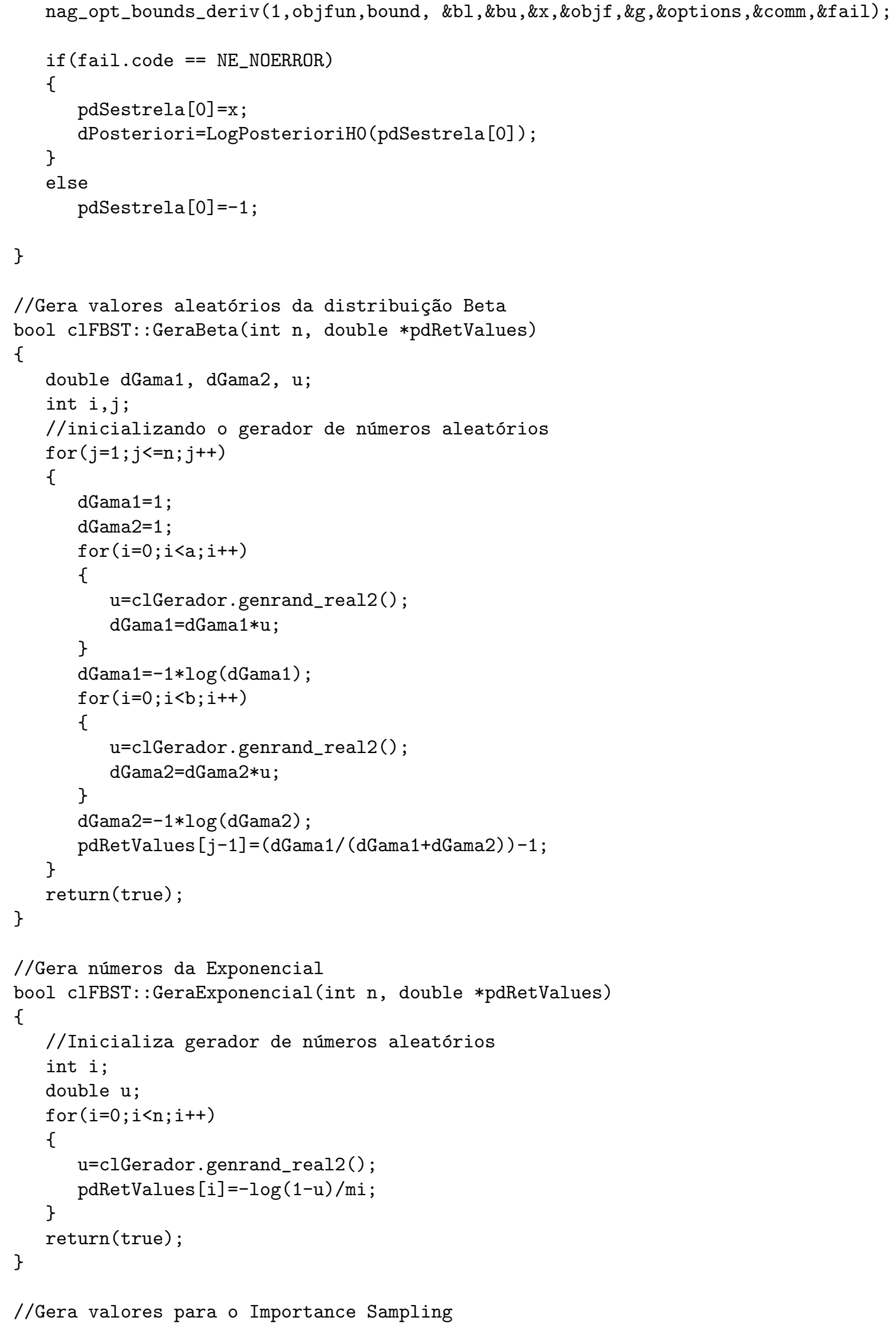




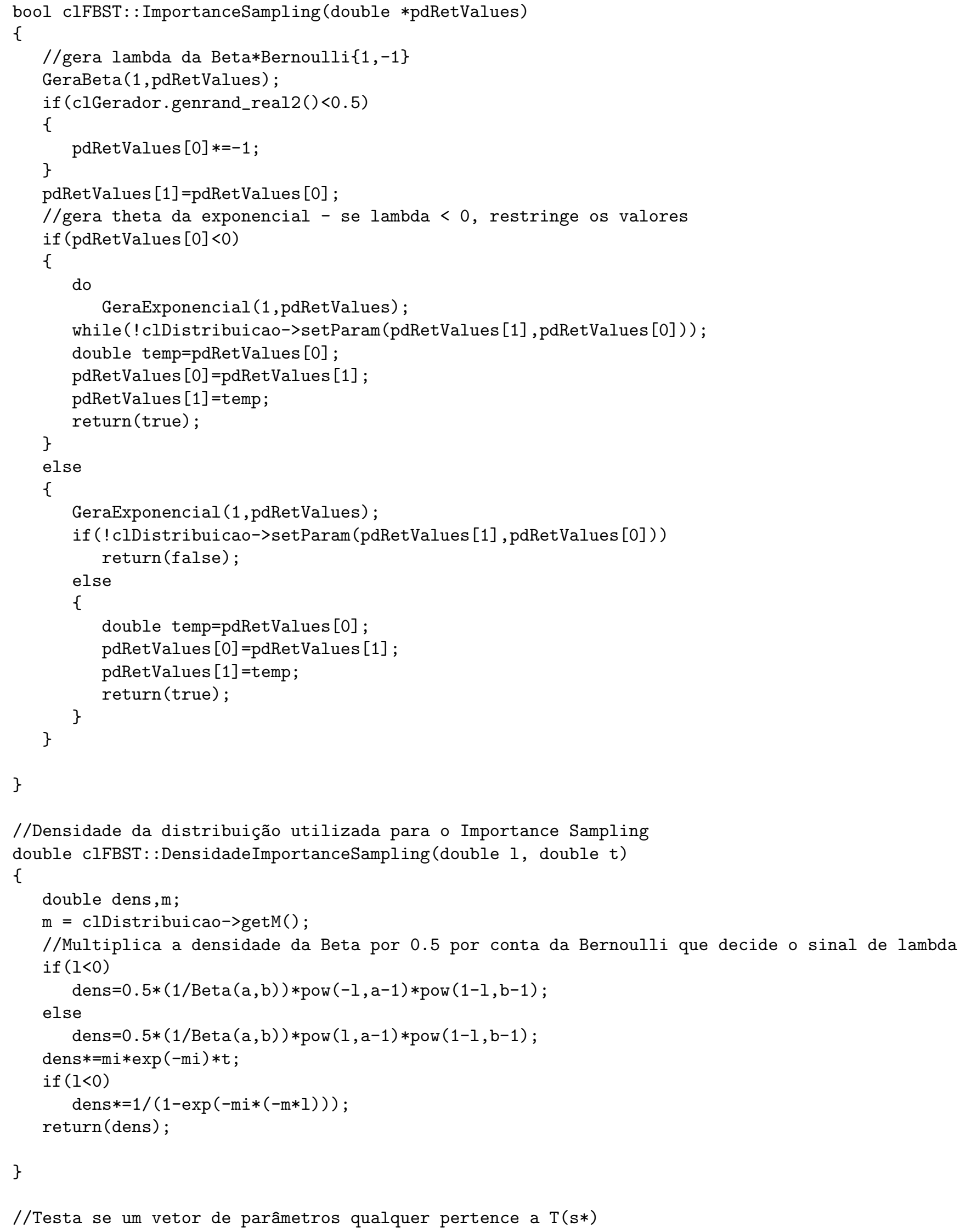




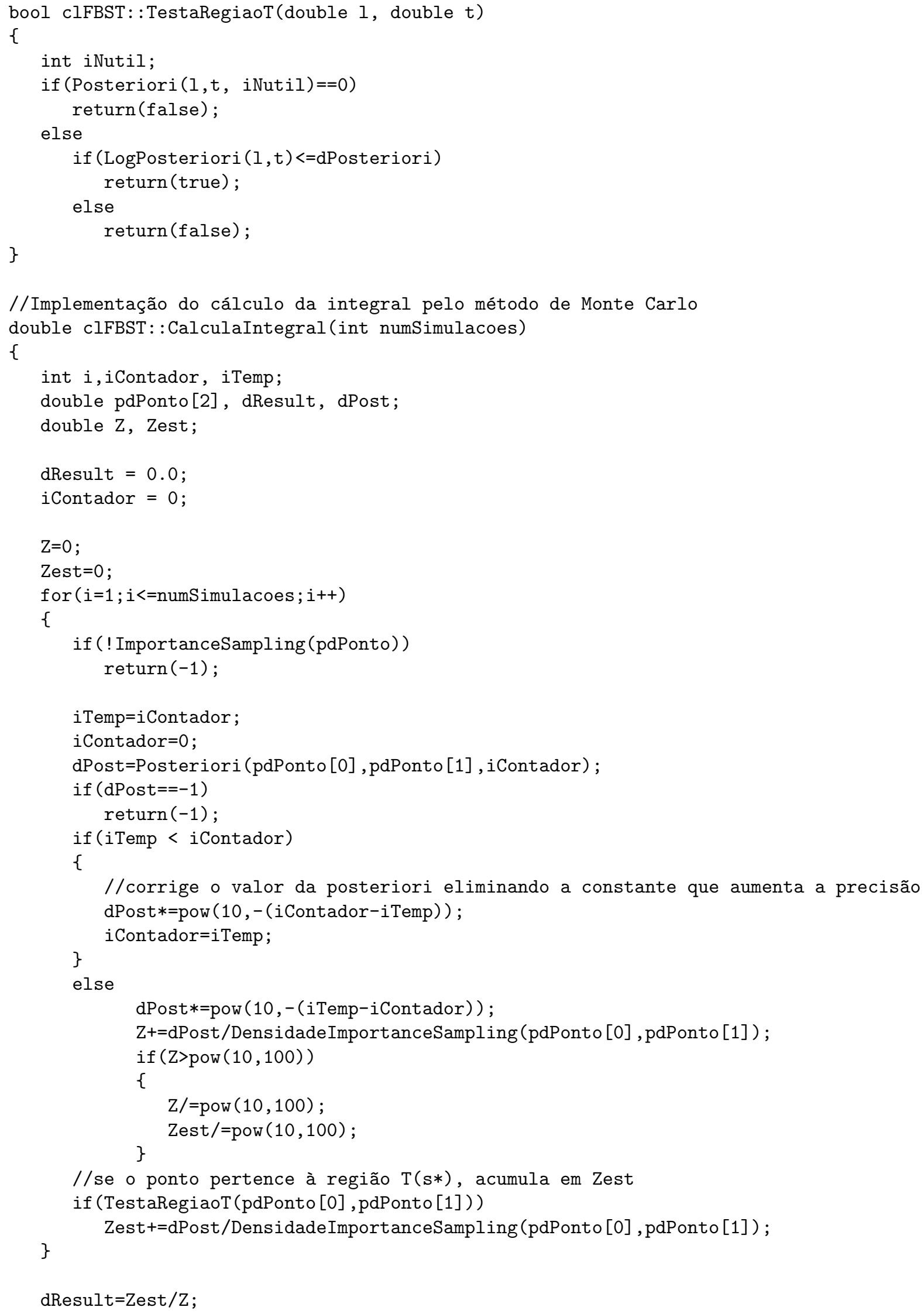


return (dResult);

\}

//Organiza o cálculo do e-valor

double clFBST: :CalculaEvidencia(int n)

\{

Calculas();

double res;

if (pdSestrela [0] ==-1)

return $(-1)$;

else

\{

res $=$ CalculaIntegral $(n)$;

return(res);

\} 


\section{Referências Bibliográficas}

[1] P.C. Consul, Generalized Poisson Distributions, first edition, CRC, Florida, 1988.

[2] C.A.B. Pereira e J.M. Stern, Evidence and credibility: full bayesian significance test for precise hypotheses, Entropy 1 (1999), 99-110.

[3] M. Greenwood e G.U.Yule, An inquiry into the nature of frequency distributions representative of multiple happenings, J. R. Stat. Soc. 83 (1920), 255-279.

[4] P.C. Consul e G.C. Jain, On the generalization of Poisson distribution, Ann. Math. Statist. 41 (1970), 1387.

[5] P.C. Consul e G.C. Jain, A generalization of the Poisson distribution, Technometrics 15 (1973), n. 4, 791-799.

[6] P.C. Consul e G.C. Jain, On some interesting properties of the generalized Poisson distribution, Biometrische 15 (1973), 495-500.

[7] P.C. Consul e M.M. Shoukri, The generalized Poisson distribution when the sample mean is larger than the sample variance, Communications in Statistics — Simulation and Computation 14 (1985), 1533-1547.

[8] F.W. Steutel e K. van Harn, Discrete analogues of selfdecomposability and stability, Ann. Probab. 7 (1979), 893-899.

[9] M. Abramowitz e I.A. Stegun, Handbook of Mathematical Functions, tenth edition, Dover, New York, 1964.

[10] P.C. Consul, A simple urn model dependent upon predetermined strategy Sankhya Ser. B 36 (1974), 391-399.

[11] J.W. Tukey, One degree of freedom for non-additivity, Biometrics 5 (1949), 232-242.

[12] P. C. Consul e L. R. Shenton, Some interesting properties of Lagrangian distributions, Comm. Stat. Theor. Meth. 2 (1973), no. 3, 263-272.

[13] P. C. Consul e M.M. Shoukri Some Chance Mechanisms Leading to a Generalized Poisson Probability Model, American Journal of Mathematical and Management Sciences 8 (1988), nos. 1 e 2, 181-202.

[14] P.C. Consul e L.R. Shenton, On the probabilistic structure and properties of discrete lagrangian distributions, A modern course of statistical distributions in scientific work 1 (1974), 41-57.

[15] R.C. Gupta, Modified power series distributions and some of its applications, Shankya Ser. B 35 (1974), 288-298.

[16] W. Feller, An introduction to probability theory and its applications, third edition, Wiley, New York, 1968.

[17] M.R. Madruga, L.G. Esteves e S. Wechsler, On the bayesianity of Pereira-Stern tests, Teste 10 (2001), núm. 2, 291-299.

[18] J. von Neumann, Various techniques used in connection with random digits, National Bureau of Standards, Applied Math Series 11 (1951), 36-38. 
[19] P.C. Consul e F. Famoye, Lagrangian probability distribution, first edition, Birkhauser, Boston, 2006.

[20] F. Famoye, Sampling from the Generalized Logarithmic Series Distribution, Computing 58 (1997), núm. 4, 365-376.

[21] H. Bolfarine e M.C. Sandoval, Introdução à inferência estatística, primeira edição, Sociedade Brasileira de Matemática, Rio de Janeiro, 2001.

[22] J. Stern, Cognitive constructivism and language, Relatório Técnico IME-USP (2006).

[23] J.M. Stern, Cognitive constructivism, Eigen-solutions, and Sharp Statistical Hypotheses, Cybernetics and Human Knowing Volume 14 (2007), Numero 1, 9-36.

[24] M. Matsumoto e T. Nishura, Mersenne twister: a 623-dimensionally equidistributed uniform pseudorandom number generator, ACM Trans. Model. Comput. Simul. 8 (1998), núm. 3, 3-30. 